\title{
Eco-Friendly Synthesis of 2-Substituted Benzothiazoles Catalyzed by Cetyltrimethyl Ammonium Bromide (CTAB) in Water
}

\author{
Xiao-Liang Yang, ${ }^{a}$ Chun-Mei Xu, ${ }^{a}$ Shao-Miao Lin, ${ }^{a}$ Jiu-Xi Chen, $*, a$ Jin-Chang Ding, ${ }^{a}$ \\ Hua-Yue Wu*,a and Wei-Ke Su${ }^{a, b}$
}

\author{
${ }^{a}$ College of Chemistry and Materials Engineering, Wenzhou University, Wenzhou, 325027, P. R. China \\ ${ }^{b}$ Zhejiang Key Laboratory of Pharmaceutical Engineering, College of Pharmaceutical Sciences, \\ Zhejiang University of Technology, 310014 Hangzhou, P. R. China
}

\begin{abstract}
Uma série de benzotiazóis 2-substituídos foi sintetizada pela condensação de 2-aminotiofenol com aldeídos (RCHO: R = Alquil, Aril, Heteroaril, 2-Arilformil) na presença de quantidade catalítica de brometo de cetiltrimetil amônio (CTAB) em água e sem adição de solventes orgânicos e oxidantes. Assim, usando esse protocolo, 2-alquilbenzotiazóis foram sintetizados em altos rendimentos e 2-arilformilbenzotiazóis foram obtidos pela condensação de 2-aminotiofenol com arilformil aldeídos pela primeira vez.
\end{abstract}

A series of 2-substituted benzothiazoles have been synthesized by the condensation of 2-aminothiophenol with aldehydes (RCHO: R = Alkyl, Aryl, Heteroaryl, 2-Arylformyl) in the presence of a catalytic amount of cetyltrimethyl ammonium bromide (CTAB) "on water" by a onepot procedure without additional organic solvents and oxidants. Thereinto, 2-alkylbenzothiazoles were synthesized in high yields and 2-arylformylbenzothiazoles were obtained from the condensation of 2-aminothiophenol with arylformyl aldehydes for the first time using the present protocol.

Keywords: 2-alkylbenzothiazole, 2-arylbenzothiazole, 2-arylformylbenzothiazole, cetyltrimethyl ammonium bromide (CTAB), water

\section{Introduction}

Benzothiazoles and their derivatives are very important group of heterocyclic bicyclic systems, ${ }^{1}$ which play a fundamental role in organic and bioorganic chemistry. They have potent antitumor activity ${ }^{2-5}$ and other important pharmaceutical utilities, ${ }^{6-9}$ such as their applications for treatment of autoimmune and inflammatory diseases, prevention of solid organ transplant rejection, epilepsy, analgesia, viral infections, cancer, and tuberculosis. ${ }^{10-16}$ Also, they can be used in industry as antioxidants and vulcanization accelerators that highlight their synthesis necessity. ${ }^{17}$

The reported methods for the synthesis of 2-substituted benzothiazole (Scheme 1) involve two major routes: the condensation of 2-aminothiophenol with aldehydes, ${ }^{18-33}$ carboxylic acids, ${ }^{6,34}$ acid chlorides, ${ }^{35}$ or esters ${ }^{36,37}$ and by the cyclization of thiobenzanilides. ${ }^{38-45}$ Some other methods

*e-mail: jiuxichen@wzu.edu.cn, huayuewu@wzu.edu.cn include microwave-mediated reaction of 2-aminothiophenol with $\beta$-chlorocinnamaldehydes, ${ }^{46}$ palladium-catalyzed Suzuki biaryl coupling of 2-halobenzothiazole with arylboronic acids, ${ }^{47,48}$ coupling of benzothiazoles with aryl bromides ${ }^{49}$ and the reaction between thiophenols and aromatic nitriles. ${ }^{50}$

However, many of these methodologies are associated with one or more disadvantages such as $(i)$ harsh reaction conditions, e.g., heating at $120{ }^{\circ} \mathrm{C}$ in xylene catalyzed by

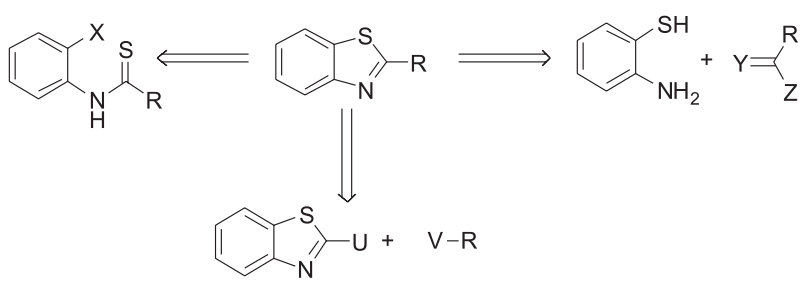

$\mathrm{X}=\mathrm{H}, \mathrm{Cl}, \mathrm{Br}, \mathrm{I}, \mathrm{SMe} ; \mathrm{Y}=\mathrm{O}, \mathrm{S}, \mathrm{Se} ; \mathrm{Z}=\mathrm{H}, \mathrm{OH}, \mathrm{OR}, \mathrm{NH}_{2}, \mathrm{Cl}$

$\mathrm{U}=\mathrm{H}, \mathrm{SnBu}_{3}, \mathrm{SnMe}_{3}, \mathrm{SMe} ; \mathrm{V}=\mathrm{COOH}, \mathrm{Br}, \mathrm{I}, \mathrm{ZnBr}$

Scheme 1. Strategies for the synthesis of 2-substituded benzothiazoles. 
4-methoxy-TEMPO in presence of oxygen; ${ }^{18}$ heating in the presence of excess of PPA at $150-220{ }^{\circ} \mathrm{C}$ for $2-4 \mathrm{~h}^{31}$ or $\mathrm{P}_{2} \mathrm{O}_{5} / \mathrm{MeSO}_{3} \mathrm{H}$ at $70{ }^{\circ} \mathrm{C}$ for $10 \mathrm{~h} ;{ }^{32}$ microwave-assisted in ionic liquids ${ }^{23}$ or using excess of $p$-TsOH adsorbed on $\mathrm{SiO}_{2} / \mathrm{K} 10$ and graphite, ${ }^{27}$ treatment with stoichiometric amounts of oxidizing agents such as $\mathrm{K}_{3} \mathrm{Fe}(\mathrm{CN})_{6}$ at $90{ }^{\circ} \mathrm{C}$ under basic conditions ${ }^{38}$ and excess of $\mathrm{Mn}(\mathrm{OAc})_{3}$ in $\mathrm{AcOH}$ at $110{ }^{\circ} \mathrm{C}$ for $4 \mathrm{~h},{ }^{41}$ (ii) prolonged reaction time, ${ }^{32,47}$ (iii) additional reagents/catalysts, high boiling solvents that are difficult to recover; ${ }^{18,21,28}(i v)$ costly, air sensitive, and toxic substances, ${ }^{18}(v)$ requirement of excess strong oxidizing agents, ${ }^{18,19,21}$ etc. In many cases the acidic/metallic wastes are generated and mixed with the effluent water. On the other hand, the condensation reaction of 2-aminothiophenol with aliphatic aldehydes tend to attract little attention. ${ }^{21,33}$ Thus, the development of environmentally benign, high yielding, and clean approaches for the synthesis of 2-substituted benzothiazoles is in demand.

The increasing concern about the tight legislation on the maintenance of greenness in synthetic processes ${ }^{51}$ led us to develop a method using a reagent that is less hazardous, non-toxic, cheap, and benign to the environment. Water as a reaction medium has gained importance in the development of sustainable chemistry. ${ }^{52}$

As a common catalyst for phase transfer, cetyltrimethyl ammonium bromide (CTAB is able to expedite the reaction between anion or nucleophile and neutral substrate via transferring one phase to another, making them collided with each other frequently. ${ }^{53}$

In continuation of our efforts to develop green synthetic routes for the formation of C-C and carbon-heteroatom bond, ${ }^{54}$ we herein report a green, simple and practical method for the synthesis of 2-sustituted benzothiazoles from the condensation of 2-aminothiophenol with aldehydes catalyzed by CTAB in water.

\section{Results and Discussion}

Initially, we investigated various conditions in the model reaction using 2-aminothiophenol with propionaldehyde in water and the results were summarized in Table 1. The results established that CTAB proved to be a superior catalyst among all the catalysts screened in this transformation. Then, the reaction was investigated with different amounts of CTAB. It was found that the yield was not significantly affected by adding amount of CTAB, 5 mol\% of CTAB was sufficient, and excessive amount of catalyst did not increase the yield remarkably (Table 1, entries 6-8).

With the optimal conditions in hand, further investigations were carried out to expand the scope of other alkyl aldehydes and the results were summarized in Table 2. In all cases, it was found that alkyl aldehydes can react well with 2-aminothiophenol in good yields without using extra oxidants. It is noteworthy that the present protocol is superior to the previous method for the synthesis of $\mathbf{3 a}$ by heating in

Table 1. The condensation of 2-aminothiophenol with propionaldehyde under different reaction conditions ${ }^{\mathrm{a}}$

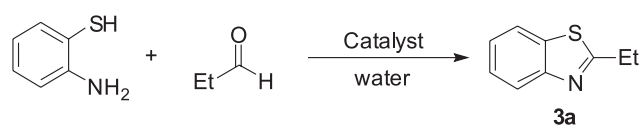

\begin{tabular}{|c|c|c|c|}
\hline Entry & Catalyst & time / h & Yields / $(\%)^{\mathrm{b}}$ \\
\hline 1 & none & 8 & 18 \\
\hline 2 & $\mathrm{SDS}^{\mathrm{c}}$ & 8 & 39 \\
\hline 3 & $\mathrm{TMAB}^{\mathrm{c}}$ & 8 & 31 \\
\hline 4 & $\mathrm{TBAF}^{\mathrm{c}}$ & 8 & 28 \\
\hline 5 & $\mathrm{TEBA}^{\mathrm{c}}$ & 8 & 41 \\
\hline 6 & CTAB & 8 & 87 \\
\hline 7 & CTAB & 8 & $89^{d}$ \\
\hline 8 & CTAB & 8 & $89^{e}$ \\
\hline
\end{tabular}

${ }^{\mathrm{a} A l l}$ reactions were run with 2 -aminobenzenethiol $(1.1 \mathrm{mmol})$, propionaldehyde ( $1 \mathrm{mmol})$, CTAB $(5 \mathrm{~mol} \%, 0.05 \mathrm{mmol})$ and water $(5 \mathrm{~mL})$ at reflux. ' ${ }^{\text {Is }}$ solated yields. 'Dodecyl sodium sulfate (SDS), Tetramethyl ammonium bromide(TMAB), Tetrabutyl ammonium fluoride(TBAF), Benzyltriethylammonium chloride(TEBA). ${ }^{\mathrm{d}}$ The load of catalyst was

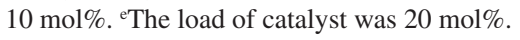

Table 2. Synthesis of 2-alkyl benzothiazoles ${ }^{\mathrm{a}}$

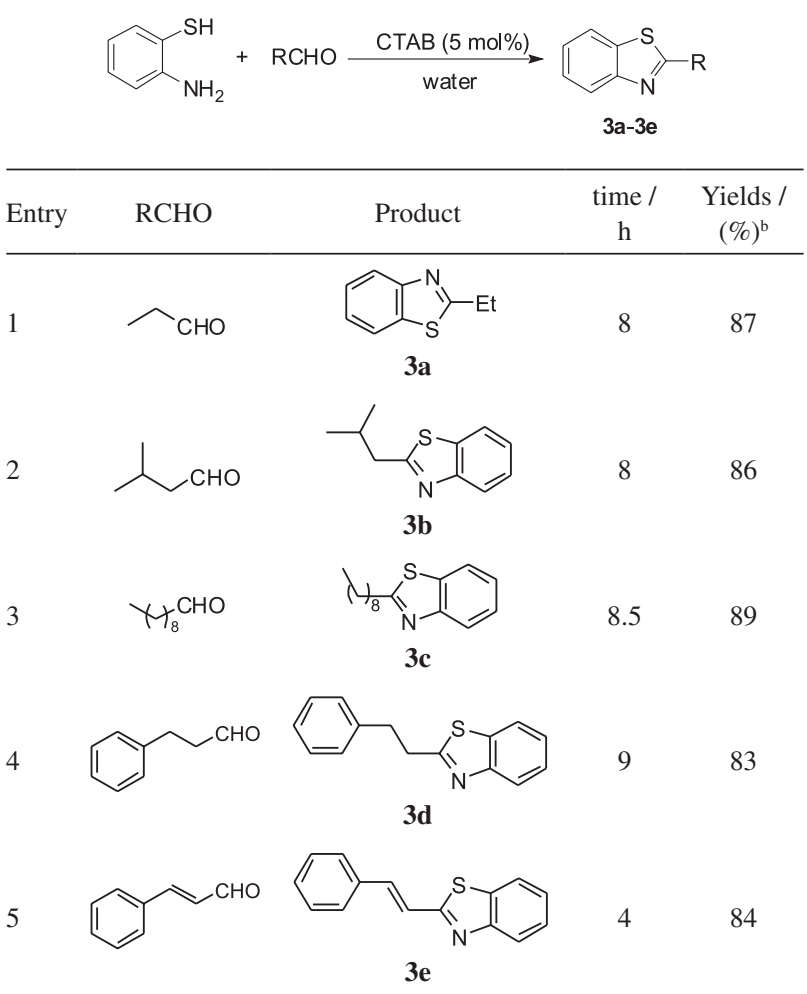

aAll reactions were run with 2-aminobenzenethiol $(1.1 \mathrm{mmol})$, alkyl aldehydes (1 mmol), CTAB ( $5 \mathrm{~mol} \%, 0.05 \mathrm{mmol})$ and water $(5 \mathrm{~mL})$ at reflux. 'Isolated yields. 
Table 3. Synthesis of 2-aryl benzothiazoles ${ }^{\mathrm{a}}$

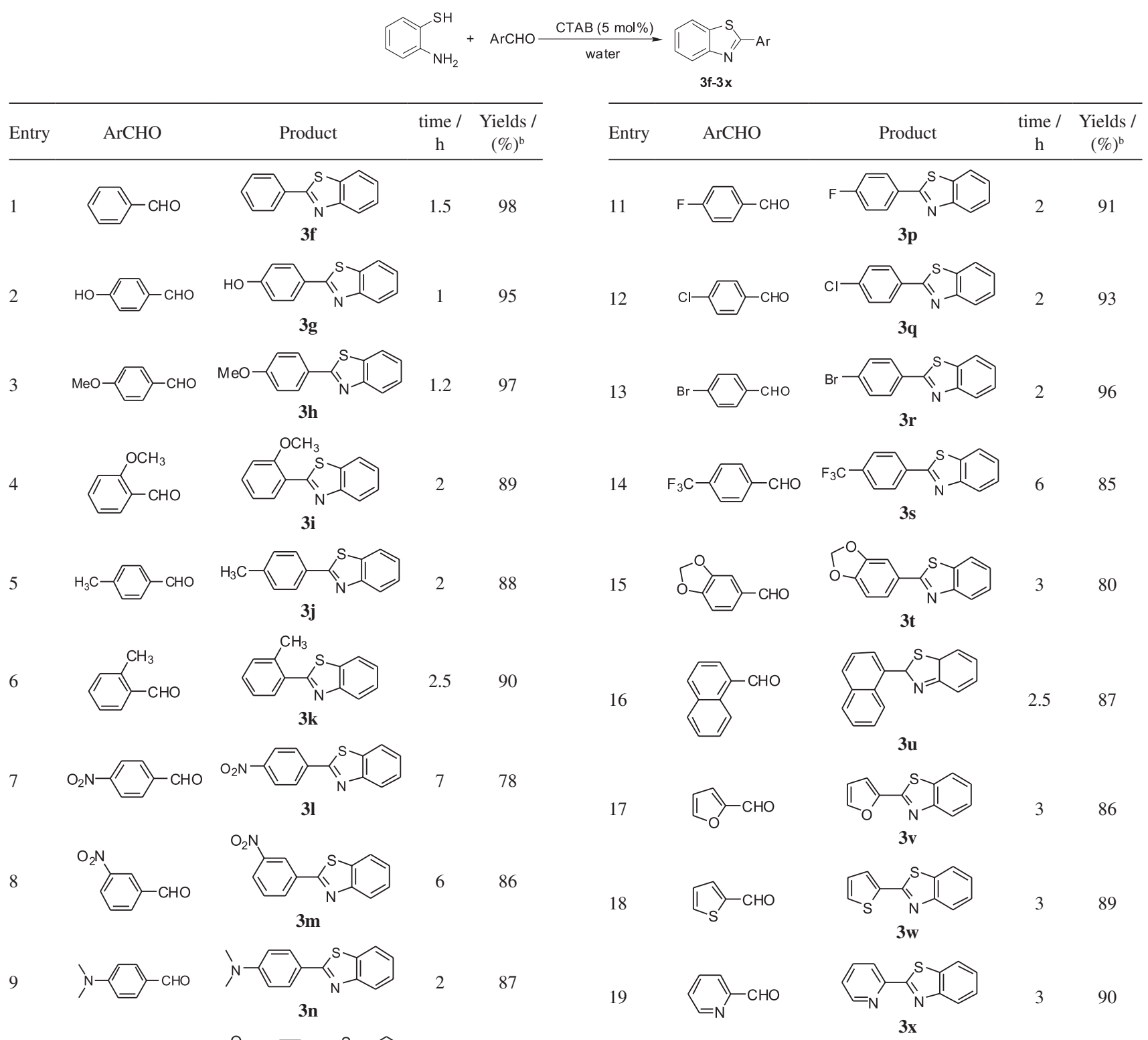

10

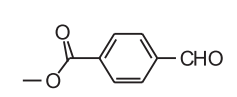

DMF at $100{ }^{\circ} \mathrm{C}$ catalyzed by $50 \mathrm{~mol} \%$ of molecular iodine ${ }^{21}$ with the yields of $31 \%$ (Table 2, entry 1). Moreover, the 2 -styrylbenzothiazole can react well with 2-aminothiophenol in high yield in short time (Table 2, entry 5).

Next, we examined the scope of the reaction of 2-aminothiophenol with a variety of aromatic aldehydes. As shown in Table 3, it was observed that a series of aromatic aldehydes bearing either electron-donating or electron-withdrawing groups on aromatic ring were investigated. The substitution groups on the aromatic ring have no obvious effect on the yields and reaction time under the above optimal conditions. However, aldehydes
${ }^{\mathrm{a} A l l}$ reactions were run with 2-aminobenzenethiol $(1.1 \mathrm{mmol})$, aryl aldehydes (1 mmol), CTAB (5 mol\%, $0.05 \mathrm{mmol})$ and water $(5 \mathrm{~mL})$ at reflux. 'Isolated yields.

with strongly electron-withdrawing groups on aromatic ring such as $p$-nitrobenzaldehyde gave the product of $\mathbf{3 l}$ with good yield in a long reaction time (Table 3, entry 7 ).

Furthermore, we also examined the condensation reaction of heteroaromatic aldehydes such as furfural, 2-thienyl aldehyde, 2-pyridyl aldehydes with 2-aminothiophenol (Table 3, entries 17-19). Similarly, the corresponding products were obtained with excellent yields.

Finally, we examined the reactivity of arylformyl aldehyde with 2-aminothiophenol in the presence of CTAB in water (Table 4). The results showed that arylformyl aldehyde exhibited analogous behavior to that of aromatic 
aldehyde and aliphatic aldehyde. To our knowledge, we reported the synthesis of 2-arylformylbenzothiazoles from the condensation of 2-aminothiophenol with arylformyl aldehyde for the first time.

Table 4. Synthesis of 2-arylformylbenzothiazoles ${ }^{\mathrm{a}}$

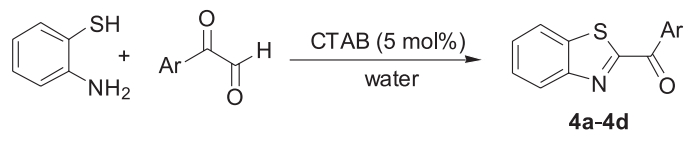

\begin{tabular}{cccc}
\hline Entry ArCOCHO & Products & $\begin{array}{c}\text { time / } \\
\mathrm{h}\end{array}$ & $\begin{array}{c}\text { Yields / } \\
(\%)^{\mathrm{b}}\end{array}$ \\
\hline
\end{tabular}

1<smiles>O=CC(=O)c1ccccc1</smiles><smiles>O=C(c1ccccc1)c1nc2ccccc2s1</smiles>

83<smiles>Cc1ccc(C(=O)C=O)cc1</smiles><smiles>O=CC(=O)c1ccc(Cl)cc1</smiles>

4

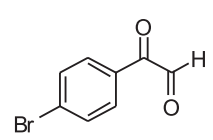<smiles>Cc1ccc(C(=O)c2nc3c(s2)CCC3)cc1</smiles>

4b

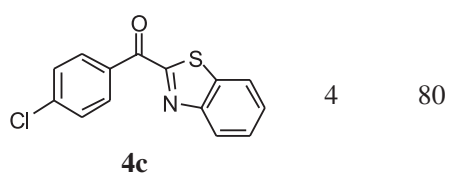

$4 c$

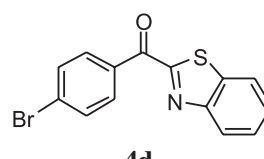

4d

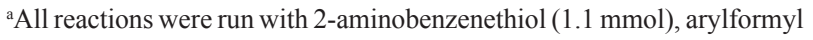
aldehydes (1 mmol), CTAB ( $5 \mathrm{~mol} \%, 0.05 \mathrm{mmol})$ and water $(5 \mathrm{~mL})$ at reflux. ${ }^{\mathrm{b}}$ Isolated yields.

A tentative mechanism for the formation of 2 -substituted benzothiazoles was proposed. It may be assumed that the bromide ion of cetyltrimethyl ammonium bromide is hydrogen-bonding to $-\mathrm{SH}$ increasing the nucleophilicity of sulfur atom, which makes the thiolate anion as a stronger nucleophile towards efficient condensation with aldehydes followed by cyclization. The second step could be a rate determining step. That the actual oxidant is the oxygen of air, wherein in the atmosphere of nitrogen resulted in an extremely sluggish reaction insufficient for complete product formation even after $10 \mathrm{~h}$. While the miceller environment formed by catalytic amount of resolved in water can accelerate the oxidation of thiazoline to thiazole.

In conclusion, we have developed a facile, efficient and green method for the synthesis of 2-substituted benzothiazoles by the condensation of alkyl, aryl, arylformyl aldehydes with 2-aminothiophenol in the presence of CTAB in water. Compared to previous reported methodologies, the present protocol features simple work-up, environmentally benign, high yields with alkyl aldehyde, no requirement of extra oxidants and use of the catalytic amounts of the cheap catalyst. Currently, studies on the extension of this protocol are ongoing in our laboratory.

\section{Experimental}

Melting points were recorded on Digital Melting Point Apparatus WRS-1B and are uncorrected. ${ }^{1} \mathrm{H}$ NMR and ${ }^{13} \mathrm{C}$ NMR spectra were taken on a Bruker DPX300 spectrometer using $\mathrm{CDCl}_{3}$ or DMSO- $d_{6}$ as the solvent with tetramethylsilane (TMS) as an internal standard at room temperature. Chemical shifts were given in $\delta$ relative to TMS, the coupling constants $J$ are given in Hz. Mass spectrometric analysis was performed on GC-MS analysis (SHIMADZU GCMS-QP2010).

\section{General procedure for the preparation of 2-substituted benzothiazoles}

To a mixture of benzaldehyde $(1 \mathrm{mmol})$ and 2-aminothiophenol (1.1 mmol), CTAB (0.05 mmol, $5 \mathrm{~mol} \%)$ was added in water $(5 \mathrm{~mL})$ under reflux. The reaction was monitored by TLC. After completion of the reaction, the product was extracted with ethyl acetate $(3 \times 10 \mathrm{~mL})$, the organic layer washed with brine $(3 \times 10 \mathrm{~mL})$, then dried over $\mathrm{Na}_{2} \mathrm{SO}_{4}$ and concentrated. The product was separated and purified by column chromatography on silica gel (300-400 mesh) using an ethyl acetate/petroleum ether mixture as the eluent to afford a pure product. When necessary, the products are purified through recrystallization from $95 \%$ ethanol.

\section{Acknowledgments}

We are grateful to the National Key Technology R\&D Program (No. 2007BAI34B00) and Natural Science Foundation of Zhejiang Province (No. Y4080107) for financial support.

\section{Supplementary Information}

Supplementary data are available free of charge at http://jbcs.sbq.org.br, as PDF file.

\section{References}

1. Bradshaw, T. D.; Wrigley, S.; Shi, D.; Schultz, R. J.; Paull, K. D.; Stevens, M. F. G.; Br. J. Cancer 1998, 77, 745; Stevens, M. F. G.; McCall, C. J.; Lelieveld, P.; Alexander, P.; Richter, A.; J. Med. Chem. 1994, 37, 1689. 
2. Bradshaw, T. D.; Westwell, A. D.; Curr. Med. Chem. 2004, 11, 1009.

3. Kashiyama, E.; Hutchinson, L.; Chua, M. S.; Stinson, S. F.; Phillips, L. R.; Kaur, G.; Sausville, E. A.; Bradshaw, T. D.; Westwell, A. D.; Stevens, M. F. G.; J. Med. Chem. 1999, 42, 4172.

4. Hutchinson, I.; Jennings, S. A.; Vishnuvajjala, B. R.; Westwell, A. D.; Stevens, M. F. G.; J. Med. Chem. 2002, 45, 744.

5. Hutchinson, I.; Chua, M. S.; Browne, H. L.; Trapani, V.; Bradshaw, T. D.; Westwell, A. D.; Stevens, M. F. G.; J. Med. Chem. 2001, 44, 1446.

6. Chen, C.; Chen, Y. J.; Tetrahedron Lett. 2004, 45, 113.

7. Tale, R. H.; Org. Lett. 2002, 4, 1641.

8. Mathis, C. A.; Wang, Y. M.; Holt, D. P.; Huang, G. F.; Debnath, M. L.; Klunk, W. E.; J. Med. Chem. 2003, 46, 2740.

9. Jackson, Y. A.; Lyon, M. A.; Townsend, N.; Bellabe, K.; Soltanik, F.; J. Chem. Soc., Perkin Trans. 1 2000, 205.

10. Das, J.; Moquin, R. V.; Liu, C.; Doweyko, A. M.; Defex, H. F.; Fang, Q.; Pang, S.; Pitt, S.; Shen, D. R.; Schieven, G. L.; Barrish, J. C.; J. Bioorg. Med. Chem. Lett. 2003, 13, 2587.

11. Hays, S. J.; Rice, M. J.; Ortwine, D. F.; Johnson, G.; Schwarz, R. D.; Boyd, D. K.; Copeland, L. F.; Vartanian, M. G.; Boxer, P. A.; J. Pharm. Sci. 1994, 83, 1425.

12. Foscolos, G.; Tsatsas, G.; Champagnac, A.; Pommier, M.; Ann. Pharm. Fr. 1977, 35, 295.

13. Shirke, V. G.; Bobad, A. S.; Bhamaria, R. P.; Khadse, B. G.; Sengupta, S. R.; Indian Drugs 1990, 27, 350.

14. Paget, C. J.; Kisner, K.; Stone, R. L.; Delong, D. C.; J. Med. Chem. 1969, 12, 1016.

15. Gong, B.; Hong, F.; Kohm, C.; Bonham, L.; Klein, P.; Bioorg. Med. Chem. Lett. 2004, 14, 1455.

16. Hutchinson, I.; Bradshaw, T. D.; Matthews, C. S.; Stevens, M. F. G.; Westwell, A. D.; Bioorg. Med. Chem. Lett. 2003, 13, 471.

17. Ivanov, S. K.; Yuritsyn, V. S.; Chem. Abstr. 1971, 74, $124487 \mathrm{~m}$.

18. Chen, Y. X.; Qian, L. F.; Zhang, W.; Han, B.; Angew. Chem., Int. Ed. 2008, 47, 9330.

19. Bahrami, K.; Khodaei, M. M.; Naali, F.; J. Org. Chem. 2008, 17,6835 .

20. Chakraborti, A. K.; Rudrawar, S.; Jadhav, K. B.; Kaur, G.; Chankeshwara, S. V.; Green Chem. 2007, 9, 1335.

21. Li, Y.; Wang, Y. L.; Wang, J. Y.; Chem. Lett. 2006, 35, 460.

22. Batista, R. M. F.; Costa, S. P. G.; Raposo, M. M. M.; Tetrahedron Lett. 2004, 45, 2825.

23. Ranu, B. C.; Jana, R.; Dey, S.; Chem. Lett. 2004, 33, 274.

24. Itoh, T.; Nagata, K.; Ishikawa, H.; Ohsawa, A.; Heterocycles 2004, 62, 197.

25. Kodomari, M.; Tamaru, Y.; Aoyama, T.; Synth. Commun. 2004, 34,3029

26. Bougrin, K.; Loupy, A.; Soufiaoui, M.; Tetrahedron 1998, 54, 8055.

27. Rostamizadeh, S.; Housaini, S. A. G.; Phosphorus, Sulfur Silicon Relat. Elem. 2005, 180, 1321.
28. Deligeorgiev, T. G.; Dyes Pigm. 1990, 12, 243.

29. Chowdhury, F. A.; Cole, E. R.; Crank, G.; J. Chromatogr. 1979, 170,73

30. Courtot, C.; Tchelitcheff, S.; Compt. Rend. 1943, 217, 231.

31. Hein, D. W.; Alheim, R. J.; Leavitt, J. J.; J. Am. Chem. Soc. 1957, 79, 427.

32. Kanaoka, Y.; Hamada, T.; Yonemitsu, O.; Chem. Pharm. Bull. 1970, 18, 587.

33. Moghaddam, F. M.; Ismaili, H.; Bardajee, G. R.; Heteroat. Chem. 2006, 17, 136.

34. Mourtas, S.; Gatos, D.; Barlos, K.; Tetrahedron Lett. 2001, 42, 2201; Njoya, Y.; Gellis, A.; Crozet, M.; Vanelle, P.; Sulfur Lett. 2003, 26, 67; Chakraborti, A. K.; Selvam, C.; Kaur, G.; Bhagat, S.; Synlett 2004, 851; Yildiz-Oren, I.; Yalcin, I.; Aki-Sener, E.; Eur. J. Med. Chem. 2004, 39, 291; Rudrawar, S.; Kondaskar, A.; Chakraborti, A. K.; Synthesis 2005, 15, 2521.

35. Laskar, I. R.; Chen, T. M.; Chem. Mater. 2004, 16, 117; Nadaf, R. N.; Siddiqui, S. A.; Daniel T.; Lahoti, R. J.; Srinivasan, K. V.; J. Mol. Catal. A: Chem. 2004, 214, 155.

36. Matsushita, H.; Lee, S. H.; Joung, M.; Clapham, B.; K. Janda, D.; Tetrahedron Lett. 2004, 45, 313.

37. Chakraborti, A. K.; Selvam, C.; Kaur, G.; Bhagat S.; Synlett 2004, 851 .

38. Hutchinson, I.; Stevens, M. F. G.; Westwel, A. D.; Tetrahedron Lett. 2000, 41, 425.

39. Benedi, C.; Bravo, F.; Uriz, P.; Fernandez, E.; Claver, C.; Castillon, S.; Tetrahedron Lett. 2003, 44, 6073.

40. Joyce, L. L.; Evindar, G.; Batey, R. A.; Chem. Commun. 2004, 446.

41. Mu, X. J.; Zou, J. P.; Zeng, R. S.; Wu, J. C.; Tetrahedron Lett. $\mathbf{2 0 0 5}, 46,4345$.

42. Moghaddam, F. M.; Boeini, H. Z.; Synlett 2005, 1612.

43. Evindar, G.; Batey, R. A.; J. Org. Chem. 2006, 71, 1802.

44. Itoh, T.; Mase, T.; Org. Lett. 2007, 9, 3687.

45. Downer-Riley, N. K.; Jackson, Y. A.; Tetrahedron 2008, 64, 7741.

46. Paul, S.; Gupta, M.; Gupta, R.; Synth.. Commun. 2002, 32, 3541.

47. Majo, V. J.; Prabhakaran, J.; Mann, J. J.; Kumar, J. S. D.; Tetrahedron Lett. 2003, 44, 8535.

48. Heo, Y.; Song, Y. S.; Kim B. T.; Heo, J. N.; Tetrahedron Lett. 2006, 47, 3091.

49. Alagille, D.; Baldwin, R. M.; Tamagnan, G. D.; Tetrahedron Lett. 2005, 46, 1349.

50. Tale, R. H.; Org. Lett. 2002, 4, 1641.

51. Tundo, P.; Anastas, P.; Black, D. S.; Breen, J.; Collins, T.; Memoli, S.; Miyamoto, J.; Polyakoff, M.; Tumas, W.; Pure Appl. Chem. 2000, 72, 1207.

52. Otto, S.; Engberts, J. B. F. N.; Pure Appl. Chem. 2000, 72, 1365; Ribe, S.; Wipf, P.; Chem. Commun. 2001, 299; Li, C. J.; Chem. Rev. 2005, 105, 3095; Narayan, S.; Muldoon, J.; Finn, M. G.; Fokin, V. V.; Kolb, H. C.; Sharpless, K. B.; Angew. Chem., Int. 
Ed.. 2005, 44, 3275; Li, C. J.; Chen, L.; Chem. Soc. Rev. 2006, 35, 68; Lindström, U. M.; Andersson, F.; Angew. Chem., Int. Ed. 2006, 45, 548; Hailes, H. C.; Org. Process Res. Dev. 2007, $11,114$.

53. Agarwal, V.; Singh, M.; McPherson, G.; John, V.; Bose, A.; Colloids Surf., A 2006, 281, 246; Li, W.; Han, Y. C.; Zhang, J. L.; Wang, B. G.; Colloid J. 2005, 67, 159; Bi, Z. C.; Liao, W.S.; Qi, L. Y.; Appl. Surf. Sci. 2004, 221, 25; Ekwall, P.; Mandell, L.; Solyom, P.; J. Colloid Interface Sci. 1971, 35, 519.

54. Chen, J. X.; Wu, H. Y.; Jin, C.; Zhang, X. X.; Xie, Y. Y.; Su, W. K.; Green Chem. 2006, 8, 330; Chen, J. X.; Wu, H. Y.; Zheng, Z. G.; Jin, C.; Zhang, X. X.; Su, W. K.; Tetrahedron Lett. 2006, 47, 5383; Su, W. K.; Chen, J. X.; Wu, H. Y.; Jin, C.; J. Org.
Chem. 2007, 72, 4524; Chen, X. A.; Zhang, C. F.; Wu, H. Y.; Yu, X. C.; Su, W. K.; Cheng, J.; Synthesis 2007, 3233; Chen, J. X.; Su, W. K.; Wu, H. Y.; Liu M. C.; Jin, C.; Green Chem. 2007, 9, 972; Chen, J. X.; Wu, D. Z.; He, F.; Liu, M. C.; Wu, H. Y.; Ding, J. C.; Su, W. K.; Tetrahedron Lett. 2008, 49, 3814; Chen, J. X.; Liu, M. C.; Yang, X. L.; Ding, J. C.; Wu, H. Y.; J. Braz. Chem. Soc. 2008, 19, 877; Zheng, H. M.; Zhang, Q.; Chen, J. X.; Liu, M. C.; Cheng, S. H.; Wu, H. Y.; Su, W. K.; J. Org. Chem. 2009, 74, 943; Xiong, W.; Chen, J. X.; Liu, M. C.; Ding, J. C.; Wu, H. Y.; Su, W. K.; J. Braz. Chem. Soc. 2009, 20, 367; Zhu, D. J.; Chen, J. X.; Liu, M. C.; Ding, J. C.; Wu, H. Y.; J. Braz. Chem. Soc. 2009, 20, 482.

Received: March 9, 2009 Web Release Date: October 9, 2009 


\title{
Eco-Friendly Synthesis of 2-Substituted Benzothiazoles Catalyzed by Cetyltrimethyl Ammonium Bromide (CTAB) in Water
}

\author{
Xiao-Liang Yang, ${ }^{a}$ Chun-Mei Xu, ${ }^{a}$ Shao-Miao Lin, ${ }^{a}$ Jiu-Xi Chen, ${ }^{*, a}$ Jin-Chang Ding, ${ }^{a}$ \\ $\mathrm{Hua}-\mathrm{Yue} \mathrm{Wu}^{*, a}$ and Wei-Ke Su${ }^{a, b}$ \\ ${ }^{a}$ College of Chemistry and Materials Engineering, Wenzhou University, Wenzhou, 325027, China \\ ${ }^{b}$ Zhejiang Key Laboratory of Pharmaceutical Engineering, College of Pharmaceutical Sciences, \\ Zhejiang University of Technology, 310014 Hangzhou, P. R. China
}

\section{Description of the Products}

2-Ethylbenzothiazole $(3 \boldsymbol{a})^{1}$<smiles>CCc1nc2ccccc2s1</smiles>

Colorless oil, ${ }^{1} \mathrm{H}$ NMR (300 $\left.\mathrm{MHz}, \mathrm{CDCl}_{3}\right) \delta 7.28-8.00$ (m, 4H, ArH), 3.10 (q, J 7.6 Hz, 2H, $\left.\mathrm{CH}_{2} \mathrm{CH}_{3}\right), 1.43$ (t, $J$ $\left.7.6 \mathrm{~Hz}, 3 \mathrm{H}, \mathrm{CH}_{2} \mathrm{CH}_{3}\right) ;{ }^{13} \mathrm{C} \mathrm{NMR}\left(75 \mathrm{MHz}, \mathrm{CDCl}_{3}\right) \delta 173.6$, $153.2,135.0,125.9,124.6,122.5,121.5,27.8,13.8 ; \mathrm{MS}$ (ESI): $m / z(\%) 164\left([\mathrm{M}+\mathrm{H}]^{+}, 100\right)$.

2-Isobutylbenzothiazole $(3 \boldsymbol{b})^{1}$

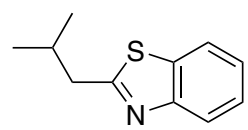

Colorless oil, ${ }^{1} \mathrm{H}$ NMR $\left(300 \mathrm{MHz}, \mathrm{CDCl}_{3}\right) \delta$ 7.98-8.01 (m, 1H, ArH), 7.84-7.87 (m, 1H, ArH), 7.43-7.48 (m, 1H, $\mathrm{ArH}), 7.35-7.38$ (m, 1H, ArH), $3.00\left(\mathrm{~d}, J 7.2 \mathrm{~Hz}, 2 \mathrm{H}, \mathrm{CH}_{2}\right)$, $2.25\left(\mathrm{~m}, 1 \mathrm{H}, \mathrm{CH}\left(\mathrm{CH}_{3}\right)_{2}\right), 1.06\left(\mathrm{~d}, J 6.6 \mathrm{~Hz}, 6 \mathrm{H}, \mathrm{CH}\left(\mathrm{CH}_{3}\right)_{2}\right)$; ${ }^{13} \mathrm{C} \mathrm{NMR}\left(75 \mathrm{MHz}, \mathrm{CDCl}_{3}\right) \delta 171.3,153.2,135.2,125.8$, 124.6, 122.5, 121.4, 43.2, 29.7, 22.4; MS (ESI): $\mathrm{m} / \mathrm{z}(\%)$ $192\left([\mathrm{M}+\mathrm{H}]^{+}, 100\right)$.

2-Nonylbenzothiazole $(3 \boldsymbol{c})^{2}$<smiles>C[As]c1nc2ccccc2s1</smiles>

Yellow oil, ${ }^{1} \mathrm{H}$ NMR $\left(300 \mathrm{MHz}, \mathrm{CDCl}_{3}\right) \delta 7.97(\mathrm{~d}, J 7.7$ $\mathrm{Hz}, 1 \mathrm{H}, \mathrm{ArH}), 7.84$ (d, J7.7Hz, 1H, ArH), 7.42-7.48 (m, 1H, $\mathrm{ArH}), 7.32-7.37$ (m, 1H, ArH), $3.12\left(\mathrm{t}, J 7.8 \mathrm{~Hz}, 2 \mathrm{H}, \mathrm{CH}_{2}\right)$; 1.83-1.93 (m, 2H, $\left.\mathrm{CH}_{2}\right), 1.27-1.47\left(\mathrm{~m}, 12 \mathrm{H},\left(\mathrm{CH}_{2}\right)_{6}\right), 0.88$ $\left(\mathrm{t}, J 6.9 \mathrm{~Hz}, 3 \mathrm{H}, \mathrm{CH}_{2} \mathrm{CH}_{3}\right) ;{ }^{13} \mathrm{C} \mathrm{NMR}\left(75 \mathrm{MHz}, \mathrm{CDCl}_{3}\right) \delta$ $172.5,153.2$, 135.1, 125.8, 124.6, 122.5, 121.5, 34.4, 31.8,

*e-mail: jiuxichen@wzu.edu.cn; huayuewu@wzu.edu.cn
29.8, 29.4, 29.3, 29.3, 29.2, 22.7, 14.1; MS (ESI): $m / z(\%)$ $262\left([\mathrm{M}+\mathrm{H}]^{+}, 100\right)$.

2-Phenethylbenzothiazole $(3 \boldsymbol{d})^{3}$

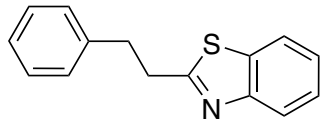

White crystal, mp 54-56 ${ }^{\circ} \mathrm{C}$ (not reported); ${ }^{1} \mathrm{H}$ NMR $\left(300 \mathrm{MHz}, \mathrm{CDCl}_{3}\right) \delta 8.02(\mathrm{~d}, J 8.0 \mathrm{~Hz}, 1 \mathrm{H}, \mathrm{ArH}) ; 7.85$ (d, $J 8.0 \mathrm{~Hz}, 1 \mathrm{H}, \mathrm{ArH}) ; 7.22-7.48(\mathrm{~m}, 6 \mathrm{H}, \mathrm{ArH}) ; 3.45$ (t, J 6.0 $\left.\mathrm{Hz}, 2 \mathrm{H}, \mathrm{CH}_{2}\right) ; 3.24\left(\mathrm{t}, J 6.0 \mathrm{~Hz}, 2 \mathrm{H}, \mathrm{CH}_{2}\right) ;{ }^{13} \mathrm{C} \mathrm{NMR}(75$ $\left.\mathrm{MHz}, \mathrm{CDCl}_{3}\right) \delta 170.9,153.1,140.1,135.1,128.6,128.4$, 126.4, 126.0, 124.7, 122.5, 121.5, 36.0, 35.5; MS (ESI): $m / z(\%) 240\left([\mathrm{M}+\mathrm{H}]^{+}, 100\right)$.

2-Styrylbenzothiazole $(3 \boldsymbol{e})^{4}$

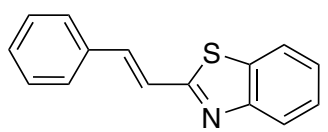

Yellow crystal, mp $110-112{ }^{\circ} \mathrm{C}$ (not reported); ${ }^{1} \mathrm{H}$ NMR $\left(300 \mathrm{MHz}, \mathrm{CDCl}_{3}\right) \delta$ 7.36-7.98 (m, $\left.11 \mathrm{H}, \mathrm{ArH}\right) ;{ }^{13} \mathrm{C} \mathrm{NMR}$ $\left(75 \mathrm{MHz}, \mathrm{CDCl}_{3}\right) \delta 166.9,153.9,137.6,135.4,134.4$, 129.4, 128.9, 127.4, 126.3, 125.3, 123.0, 122.2, 121.5; MS (ESI): $m / z(\%) 238\left([\mathrm{M}+\mathrm{H}]^{+}, 100\right)$.

2-Phenylbenzothiazole $(3 \boldsymbol{f})^{4}$

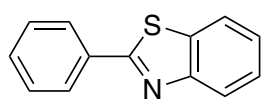

White solid, mp $111-112{ }^{\circ} \mathrm{C}\left(113-114{ }^{\circ} \mathrm{C}\right)^{4} ;{ }^{1} \mathrm{H}$ NMR (300 $\left.\mathrm{MHz}, \mathrm{CDCl}_{3}\right) \delta 8.10-8.12$ (m, 3H, ArH), 7.91(d, J $7.7 \mathrm{~Hz}, 1 \mathrm{H}, \mathrm{ArH}), 7.48-7.53$ (m, 4H, ArH), 7.40 (d, J 7.7 $\mathrm{Hz}, 1 \mathrm{H}, \mathrm{ArH}) .{ }^{13} \mathrm{C} \mathrm{NMR}\left(75 \mathrm{MHz}, \mathrm{CDCl}_{3},\right) \delta 168.1,154.1$, 135.0, 133.6, 131.0, 129.1, 127.6, 126.3, 125.2, 123.2, 121.6; MS (ESI): $m / z(\%) 212\left([\mathrm{M}+\mathrm{H}]^{+}, 100\right)$. 
2-(4-Hydroxyphenyl) benzothiazole $(\mathbf{3 g})^{4}$<smiles>Oc1ccc(-c2nc3ccccc3s2)cc1</smiles>

White solid, mp $229-231{ }^{\circ} \mathrm{C}\left(227-228{ }^{\circ} \mathrm{C}\right)^{4} ;{ }^{1} \mathrm{H}$ NMR $\left(300 \mathrm{MHz}\right.$, DMSO- $\left.d_{6}\right) \delta 7.89-8.06$ (m, 4H, ArH), 7.36-7.50 (m, 2H, ArH), 6.90-6.95(m, 2H, ArH), 3.64 (br s, 1H, OH); ${ }^{13} \mathrm{C}$ NMR $\left(75 \mathrm{MHz}, \mathrm{CDCl}_{3},\right) \delta 167.9,170.1,154.1,134.5$, 129.5, 126.8, 125.3, 124.4, 122.7, 122.5, 116.5; MS (ESI): $\mathrm{m} / \mathrm{z}(\%) 228\left([\mathrm{M}+\mathrm{H}]^{+}, 100\right)$.

2-(4-Methoxyphenyl)benzothiazole $(\mathbf{3 h})^{5}$<smiles>COc1ccc(-c2nc3ccccc3s2)cc1</smiles>

Yellow crystal, mp $120-121{ }^{\circ} \mathrm{C}\left(119-121^{\circ} \mathrm{C}\right)^{5} ;{ }^{1} \mathrm{H}$ NMR $\left(300 \mathrm{MHz}, \mathrm{CDCl}_{3}\right) \delta$ 8.04-8.07 (m, 3H, ArH), $7.90(\mathrm{~m}$, $1 \mathrm{H}, \mathrm{ArH}), 7.48(\mathrm{~d}, J 7.3 \mathrm{~Hz}, 1 \mathrm{H}, \mathrm{ArH}), 7.37$ (d, $J 7.3 \mathrm{~Hz}$, 1H, ArH), 7.01-7.04 (m, 2H, ArH), 3.90 (s, 3H, $\mathrm{OCH}_{3}$ ); ${ }^{13} \mathrm{C}$ NMR $\left(75 \mathrm{MHz}, \mathrm{CDCl}_{3}\right) \delta 168.0,162.1,154.4,135.1$, 129.3, 126.6, 126.4, 125.0, 123.0, 121.7, 114.5, 55.6; MS (ESI): $m / z(\%) 242\left([\mathrm{M}+\mathrm{H}]^{+}, 100\right)$.

2-(2-Methoxyphenyl)-benzothiazole $(3 i)^{1}$<smiles>COc1ccccc1-c1nc2ccccc2s1</smiles>

White crystal, mp $120-122{ }^{\circ} \mathrm{C}$ (not reported); ${ }^{1} \mathrm{H}$ NMR (300 MHz, $\mathrm{CDCl}_{3}$ ) $\delta$ 7.03-8.58 (m, 8H, ArH), 4.03 (s, 3H, $\left.\mathrm{OCH}_{3}\right) ;{ }^{13} \mathrm{C} \mathrm{NMR}\left(75 \mathrm{MHz}, \mathrm{CDCl}_{3}\right) \delta 162.8,156.9,151.9$, 135.8, 131.4, 129.2, 125.6, 124.3, 122.5, 120.9, 120.8, 111.4, 55.4; MS (ESI): $m / z(\%) 242\left([\mathrm{M}+\mathrm{H}]^{+}, 100\right)$.

2-(4-Methylphenyl)-benzothiazole $(\mathbf{3 j})^{4}$<smiles>Cc1ccc(-c2nc3ccccc3s2)cc1</smiles>

Yellow crystal, mp $85-86^{\circ} \mathrm{C}\left(84-85^{\circ} \mathrm{C}\right)^{4} ;{ }^{1} \mathrm{H}$ NMR $(300$ $\mathrm{MHz}, \mathrm{CDCl}_{3}$ ) $\delta 8.05$ (d, J 8.0 Hz, 1H, ArH), 7.97 (d, J 8.0 $\mathrm{Hz}, 2 \mathrm{H}, \mathrm{ArH}), 7.86$ (d, J 8.0 Hz, 1H, ArH), 7.44-7.46 (m, 1H, ArH), 7.34-7.37 (m, 1H, ArH), 7.27 (d, J 8.0 Hz, 1H, $\mathrm{ArH}), 2.40\left(\mathrm{~s}, 3 \mathrm{H}, \mathrm{CH}_{3}\right) ;{ }^{13} \mathrm{CNMR}\left(75 \mathrm{MHz}, \mathrm{CDCl}_{3}\right) \delta 168.2$, 154.2, 141.4, 135.0, 131.0, 129.7, 127.5, 126.2, 125.0, 123.0, 121.5, 21.5; MS (ESI): $m / z(\%) 226\left([\mathrm{M}+\mathrm{H}]^{+}, 100\right)$.
2-(2-Methylphenyl)-benzothiazole $(3 \boldsymbol{k})^{1}$<smiles>Cc1ccccc1-c1nc2ccccc2s1</smiles>

White crystal, mp 53-54 ${ }^{\circ} \mathrm{C}\left(53-54{ }^{\circ} \mathrm{C}\right) ;{ }^{1} \mathrm{H}$ NMR (300 $\mathrm{MHz}, \mathrm{CDCl}_{3}$ ) $\delta 8.1$ (d, J $\left.8.0 \mathrm{~Hz}, 1 \mathrm{H}, \mathrm{ArH}\right), 7.90$ (d, J 8.0 $\mathrm{Hz}, 1 \mathrm{H}, \mathrm{ArH}), 7.75$ (d, J 7.6 Hz, 1H, ArH), 7.50 (d, J 7.6 $\mathrm{Hz}, 1 \mathrm{H}, \mathrm{ArH}), 7.26-7.41$ (m, 4H, $\mathrm{ArH}), 2.65$ (s, 3H, $\mathrm{CH}_{3}$ ); ${ }^{13} \mathrm{C} \mathrm{NMR}\left(75 \mathrm{MHz}, \mathrm{CDCl}_{3}\right) \delta 163.1,157.2,152.1,136.1$, 131.7, 129.5, 125.8, 124.5, 122.7, 122.2, 121.2, 121.1, 111.6, 55.6; MS (ESI): $m / z(\%) 226\left([\mathrm{M}+\mathrm{H}]^{+}, 100\right)$.

2-(4-Nitrophenyl)-benzothiazole $(3 l)^{5}$<smiles>O=[N+]([O-])c1ccc(-c2nc3ccccc3s2)cc1</smiles>

Yellow crystal, mp $231-232^{\circ} \mathrm{C}\left(229-230{ }^{\circ} \mathrm{C}\right)^{5} ;{ }^{1} \mathrm{H}$ NMR (300 MHz, $\left.\mathrm{CDCl}_{3}\right) \delta 7.46-8.38$ (m, 8H, ArH); ${ }^{13} \mathrm{C}$ NMR (75 $\left.\mathrm{MHz}, \mathrm{CDCl}_{3}\right) \delta 164.8,154.1,149.0,139.1,135.5,128.2$, 126.9, 126.2, 124.3, 123.9, 121.8; MS (ESI): $\mathrm{m} / \mathrm{z}$ (\%) 257 $\left([\mathrm{M}+\mathrm{H}]^{+}, 100\right)$.

\section{2-(3-Nitrophenyl)-benzothiazole $(3 \mathbf{m})^{6}$ \\ $\mathrm{O}_{2} \mathrm{~N}$<smiles>Cc1cccc(-c2nc3ccccc3s2)c1</smiles>

Yellow crystal, mp $185-186^{\circ} \mathrm{C}\left(183-185^{\circ} \mathrm{C}\right)^{6} ;{ }^{1} \mathrm{H}$ NMR $\left(300 \mathrm{MHz}, \mathrm{CDCl}_{3}\right) \delta$ 7.43-8.95 (m, 8H, ArH); ${ }^{13} \mathrm{C}$ NMR (75 $\left.\mathrm{MHz}, \mathrm{CDCl}_{3}\right) \delta 164.8,153.8,148.6,135.2,135.1,132.9$, 130.0, 126.8, 126.0, 125.1, 123.7, 122.2, 121.8; MS (ESI): $\mathrm{m} / \mathrm{z}(\%) 257\left([\mathrm{M}+\mathrm{H}]^{+}, 100\right)$.

\section{2-(4-N,N-dimethylaminophenyl)benzothiazole $(3 \boldsymbol{n})^{4}$}<smiles>CN(C)c1ccc(-c2nc3ccccc3s2)cc1</smiles>

Brown crystal, mp $173-175^{\circ} \mathrm{C}\left(176-178{ }^{\circ} \mathrm{C}\right)^{4} ;{ }^{1} \mathrm{H}$ NMR $\left(300 \mathrm{MHz}, \mathrm{CDCl}_{3}\right) \delta 7.98(\mathrm{dd}, J 7.0 \mathrm{~Hz}, J 2.0 \mathrm{~Hz}, 3 \mathrm{H}$, ArH), 7.83-7.86 (m, 1H, ArH), 7.42-7.45 (m, 1H, ArH), 7.3.-7.33 (m, 1H, ArH), 6.74 (dd, J $2.0 \mathrm{~Hz}, J 7.0 \mathrm{~Hz}, 2 \mathrm{H}$, $\mathrm{ArH}), 3.05$ (s, 6H, N( $\left.\left.\mathrm{CH}_{3}\right)_{2}\right) ;{ }^{13} \mathrm{C} \mathrm{NMR}\left(75 \mathrm{MHz}, \mathrm{CDCl}_{3}\right.$ ) $\delta 168.8,154.4,152.1,134.5,128.8,125.9,124.1,122.2$, 121.3, 111.6, 40.1; MS (ESI): $m / z(\%) 255\left([\mathrm{M}+\mathrm{H}]^{+}, 100\right)$. 
2-(4-Methoxycarbonylphenyl) benzothiazole $(30)^{7}$<smiles>COC(=O)c1ccc(-c2nc3ccccc3s2)cc1</smiles>

White crystal, mp $166-167{ }^{\circ} \mathrm{C}\left(166^{\circ} \mathrm{C}\right)^{7} ;{ }^{1} \mathrm{H}$ NMR $(300$ $\left.\mathrm{MHz}, \mathrm{CDCl}_{3}\right) \delta$ 8.07-8.12 (m, 5H, ArH), 7.87-7.89 (m, $1 \mathrm{H}, \mathrm{ArH}), 7.39-7.50$ (m, 2H, ArH), 3.94 (s, 3H, $\mathrm{OCH}_{3}$ ); ${ }^{13} \mathrm{C}$ NMR $\left(75 \mathrm{MHz}, \mathrm{CDCl}_{3}\right) \delta 166.4,166.3,150.4,137.3$, 135.2, 131.9, 130.1, 127.3, 126.5, 125.6, 123.5, 121.6, 52.25; MS (ESI): $m / z(\%) 270\left([\mathrm{M}+\mathrm{H}]^{+}, 100\right)$.

2-(4-Fluorophenyl)benzothiazole $(3 \boldsymbol{p})^{5}$<smiles>Fc1ccc(-c2nc3ccccc3s2)cc1</smiles>

White crystal, mp $98-100{ }^{\circ} \mathrm{C}\left(98-99{ }^{\circ} \mathrm{C}\right){ }^{5} ;{ }^{1} \mathrm{H}$ NMR (300 MHz, $\left.\mathrm{CDCl}_{3}\right) \delta$ 8.03-8.07 (m, 3H, ArH), 7.85 (d, $J$ $8.0 \mathrm{~Hz}, 1 \mathrm{H}, \mathrm{ArH}), 7.47(\mathrm{~d}, J 7.7 \mathrm{~Hz}, 1 \mathrm{H}, \mathrm{ArH}), 7.35$ (d, $J$ $7.7 \mathrm{~Hz}, 1 \mathrm{H}, \mathrm{ArH}), 7.15$ (t, $J 8.0 \mathrm{~Hz}, 1 \mathrm{H}, \mathrm{ArH}) ;{ }^{13} \mathrm{C} \mathrm{NMR}$ $\left(75 \mathrm{MHz}, \mathrm{CDCl}_{3}\right) \delta 166.9,163.0\left(\mathrm{~d},{ }^{1} J_{C-F} 250.3 \mathrm{~Hz}\right), 154.3$, 135.2, 130.1, $129.7\left(\mathrm{~d},{ }^{3} J_{C-F} 7.0 \mathrm{~Hz}\right), 129.6,125.4,123.4$ $\left(\mathrm{d},{ }^{4} J_{C-F} 2.9 \mathrm{~Hz}\right), 121.7,116.3\left(\mathrm{~d},{ }^{2} J_{C-F}=22.0 \mathrm{~Hz}\right) ; \mathrm{MS}(\mathrm{ESI})$ : $\mathrm{m} / z(\%) 230\left([\mathrm{M}+\mathrm{H}]^{+}, 100\right)$.

2-(4-Chlorophenyl)benzothiazole $(\mathbf{3 q})^{8}$<smiles>Clc1ccc(-c2nc3ccccc3s2)cc1</smiles>

Yellow crystal, mp $113-114^{\circ} \mathrm{C}\left(113^{\circ} \mathrm{C}\right)^{8} ;{ }^{1} \mathrm{H}$ NMR $(300$ $\left.\mathrm{MHz}, \mathrm{CDCl}_{3}\right) \delta$ 7.87-8.06 (m, 4H, ArH), 7.35-7.51 (m, 4H, $\mathrm{ArH}) .{ }^{13} \mathrm{C}$ NMR (75 MHz, $\left.\mathrm{CDCl}_{3}\right) \delta 166.8,154.3,137.2$, 135.3, 132.3, 129.5, 128.9, 126.7, 125.6, 123.5, 121.8; MS (ESI): $m / z(\%) 246\left([\mathrm{M}+\mathrm{H}]^{+}, 100\right), 248\left([\mathrm{M}+2+\mathrm{H}]^{+}, 35\right)$.

2-(4-Bromophenyl)benzothiazole $(3 r)^{5}$<smiles>Brc1ccc(-c2nc3ccccc3s2)cc1</smiles>

Yellow crystal, mp $131-132{ }^{\circ} \mathrm{C}\left(130-131{ }^{\circ} \mathrm{C}\right)^{5} ;{ }^{1} \mathrm{H}$ NMR $\left(300 \mathrm{MHz}, \mathrm{CDCl}_{3}\right) \delta 7.37-8.08$ (m, 8H, ArH); ${ }^{13} \mathrm{C} \mathrm{NMR}(75$ $\left.\mathrm{MHz}, \mathrm{CDCl}_{3}\right) \delta 166.7,154.1,135.0,132.5,132.2,128.9$, 126.5, 125.5, 125.4, 123.3, 121.6; MS (ESI): $\mathrm{m} / z$ (\%) 291 $\left([\mathrm{M}+\mathrm{H}]^{+}, 100\right), 289\left([\mathrm{M}+2+\mathrm{H}]^{+}, 97\right)$.
2-(4-(Trifluoromethyl) phenyl) benzothiazole $(3 \mathrm{~s})^{9}$<smiles>FC(F)(F)c1ccc(-c2nc3ccccc3s2)cc1</smiles>

Yellow crystal, mp $160-162{ }^{\circ} \mathrm{C}$ (not reported); ${ }^{1} \mathrm{H}$ NMR $\left(300 \mathrm{MHz}, \mathrm{CDCl}_{3}\right) \delta$ 8.08-8.18 (m, 3H, $\left.\mathrm{ArH}\right), 7.90(\mathrm{~d}$, $J 8.1 \mathrm{~Hz}, 1 \mathrm{H}, \mathrm{ArH}), 7.70(\mathrm{~d}, J 8.1 \mathrm{~Hz}, 2 \mathrm{H}, \mathrm{ArH}), 7.39-$ 7.7.54 (m, $2 \mathrm{H}, \mathrm{ArH}) ;{ }^{13} \mathrm{C}$ NMR (75 MHz, $\left.\mathrm{CDCl}_{3}\right) \delta 165.9$, $153.9,136.6,135.1,132.3\left(\mathrm{q},{ }^{2} J_{C-F} 32.8 \mathrm{~Hz}, C-\mathrm{CF}_{3}\right), 127.7$, $126.6,125.8\left(\mathrm{q},{ }^{3} J_{C-F} 3.8 \mathrm{~Hz}, \mathrm{CH}-\mathrm{C}-\mathrm{CF}_{3}\right), 125.7,124.6(\mathrm{q}$, $\left.{ }^{1} J_{C-F} 270.1 \mathrm{~Hz}, \mathrm{CF}_{3}\right), 123.5,121.7$; MS (ESI): $\mathrm{m} / z$ (\%) 280 $\left([\mathrm{M}+\mathrm{H}]^{+}, 100\right)$.

\section{2-(Benzo[1, 3] dioxol-5-yl)benzothiazole $(3 t)^{4}$}

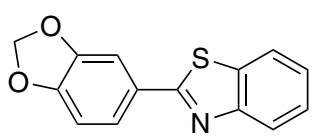

Yellow crystal, mp $125-127^{\circ} \mathrm{C}\left(127-128^{\circ} \mathrm{C}\right)^{4} ;{ }^{1} \mathrm{H}$ NMR $\left(300 \mathrm{MHz}, \mathrm{CDCl}_{3}\right) \delta 8.04(\mathrm{~d}, J 8.1 \mathrm{~Hz}, 1 \mathrm{H}, \mathrm{ArH}), 7.90$ (d, $J 8.1 \mathrm{~Hz}, 1 \mathrm{H}, \mathrm{ArH}), 7.60-7.63(\mathrm{~m}, 2 \mathrm{H}, \mathrm{ArH}), 7.46-7.49$ (m, 1H, ArH), 7.38-7.41 (m, 1H, ArH), 7.00 (d, J 7.8 Hz, 1H, $\mathrm{ArH}), 6.08$ (s, $\left.2 \mathrm{H}, \mathrm{OCH}_{2} \mathrm{O}\right) ;{ }^{13} \mathrm{C} \mathrm{NMR}\left(75 \mathrm{MHz}, \mathrm{CDCl}_{3}\right)$ $\delta$ 167.5, 154.1, 150.1, 148.4, 134.9, 128.0, 126.2, 124.9, 122.9, 122.5, 121.5, 108.6, 107.5, 101.7; MS (ESI): $\mathrm{m} / \mathrm{z}$ (\%) $256\left([\mathrm{M}+\mathrm{H}]^{+}, 100\right)$.

\section{2-(Naphthalen-1-yl)benzothiazole $(\mathbf{3} \boldsymbol{u})^{4}$}<smiles>C1=CC2=NC(c3cccc4ccccc34)SC2C=C1</smiles>

White crystal, mp $125-127^{\circ} \mathrm{C}\left(126^{\circ} \mathrm{C}\right)^{4} ;{ }^{1} \mathrm{H}$ NMR $(300$ $\left.\mathrm{MHz}, \mathrm{CDCl}_{3}\right) \delta 8.97(\mathrm{~d}, J 8.3 \mathrm{~Hz}, 1 \mathrm{H}, \mathrm{ArH}), 8.23$ (d, J 7.8 $\mathrm{Hz}, 1 \mathrm{H}, \mathrm{ArH}), 7.95-8.03$ (m, 4H, ArH), 7.58-7.63 (m, 4H, $\mathrm{ArH}), 7.48-7.56(\mathrm{~m}, 1 \mathrm{H}, \mathrm{ArH}) ;{ }^{13} \mathrm{C} \mathrm{NMR}\left(75 \mathrm{MHz}, \mathrm{CDCl}_{3}\right)$ $\delta$ 167.7, 154.2, 135.5, 134.1, 131.1, 130.9, 130.7, 129.5, 128.5, 127.7, 126.6, 126.3, 126.0, 125.3, 125.0, 123.6, 121.5; MS (ESI): $m / z(\%) 264\left([\mathrm{M}+\mathrm{H}]^{+}, 100\right)$.

2-(Furan-2-yl)benzothiazole $(3 v)^{4}$<smiles>c1coc(-c2nc3ccccc3s2)c1</smiles>

Yellow crystal, mp $102-104{ }^{\circ} \mathrm{C}\left(103{ }^{\circ} \mathrm{C}\right)^{4} ;{ }^{1} \mathrm{H}$ NMR $\left(300 \mathrm{MHz}, \mathrm{CDCl}_{3}\right) \delta 8.05(\mathrm{~d}, J 8.1 \mathrm{~Hz}, 1 \mathrm{H}, \mathrm{ArH}), 7.90(\mathrm{~d}$, $J 8.1 \mathrm{~Hz}, 1 \mathrm{H}, \mathrm{ArH}), 7.60$ (s, 1H, ArH), 7.46-7.51 (m, 1H, ArH), 7.35-7.40 (m, 1H, ArH), 7.18-7.19 (m, 1H, ArH), 6.59-6.60(m, 1H, ArH); ${ }^{13} \mathrm{C}$ NMR (75 MHz, $\left.\mathrm{CDCl}_{3}\right) \delta$ 157.7, 153.9, 140.9, 144.9, 134.5, 126.6, 125.3, 123.3, 121.7, 112.7, 111.6; MS (ESI): $m / z(\%) 202\left([\mathrm{M}+\mathrm{H}]^{+}, 100\right)$. 
2-(Thiophen-2-yl)benzothiazole $(3 w)^{l}$<smiles>c1csc(-c2nc3ccccc3s2)c1</smiles>

White crystal, mp 99-100 ${ }^{\circ} \mathrm{C}\left(99^{\circ} \mathrm{C}\right)^{1} ;{ }^{1} \mathrm{H}$ NMR $\left(300 \mathrm{MHz}, \mathrm{CDCl}_{3}\right) \delta$ 8.02-8.05 (m, 1H, ArH), 7.84-7.87 (m, 1H, ArH), 7.66 (dd, J3.6 Hz, J 1.2 HZ, 1H, ArH), 7.48$7.52(\mathrm{~m}, 2 \mathrm{H}, \mathrm{ArH}), 7.37-7.39(\mathrm{~m}, 1 \mathrm{H}, \mathrm{ArH}), 7.14(\mathrm{~m}, 1 \mathrm{H}$, $\mathrm{ArH}) ;{ }^{13} \mathrm{C} \mathrm{NMR}\left(75 \mathrm{MHz}, \mathrm{CDCl}_{3}\right) \delta 161.4,153.7,137.3$, 134.7, 129.3, 128.6, 128.0, 126.4, 125.2, 122.9, 121.4; MS (ESI): $m / z(\%) 218\left([\mathrm{M}+\mathrm{H}]^{+}, 100\right)$.

2-(Pyridin-2-yl) benzothiazole $(3 \boldsymbol{x})^{4}$<smiles>c1ccc(-c2nc3ccccc3s2)nc1</smiles>

White crystal, mp $136-137^{\circ} \mathrm{C}\left(136-137^{\circ} \mathrm{C}\right)^{4}$; ${ }^{1} \mathrm{H}$ NMR $\left(300 \mathrm{MHz}, \mathrm{CDCl}_{3}\right) \delta 8.64-8.65(\mathrm{~m}, 1 \mathrm{H}, \mathrm{ArH}), 8.32-8.35$ (m, 1H, ArH), 8.05-8.08 (m, 1H, ArH), 7.91-7.93 (m, 1H, ArH), 7.76-7.79 (m, 1H, ArH), 7.32-7.47 (m, 2H, ArH); ${ }^{13} \mathrm{C}$ NMR $\left(75 \mathrm{MHz}, \mathrm{CDCl}_{3},\right) \delta 169.5,154.5,151.6,149.8$, 137.1, 136.3, 126.4, 125.8, 125.4, 123.8, 122.2, 120.9; MS (ESI): $m / z(\%) 213\left([\mathrm{M}+\mathrm{H}]^{+}, 100\right)$.

2-Benzoylbenzothiazole $(\mathbf{4 a})^{10}$<smiles>O=C(c1ccccc1)c1nc2ccccc2s1</smiles>

Yellow crystal, mp 98-99 ${ }^{\circ} \mathrm{C}\left(98-99{ }^{\circ} \mathrm{C}\right){ }^{10} ;{ }^{1} \mathrm{H}$ NMR (300 MHz, $\left.\mathrm{CDCl}_{3}\right) \delta 8.55-8.59$ (m, 2H, ArH), 8.24-8.27 (m, 1H, ArH), 8.02-8.05 (m, 1H, ArH), 7.54-7.71 (m, 5H, $\mathrm{ArH}) ;{ }^{13} \mathrm{C}$ NMR $\left(75 \mathrm{MHz}, \mathrm{CDCl}_{3}\right) \delta 185.7,167.4,154.2$, 137.3, 135.3, 134.2, 131.6, 128.9, 128.0, 127.3, 126.1, 122.5; MS (ESI): $m / z(\%) 240\left([\mathrm{M}+\mathrm{H}]^{+}, 100\right)$.

2-(4-Methylbenzoyl) benzothiazole $(\mathbf{4 b})^{11}$<smiles>Cc1ccc(C(=O)c2nc3ccccc3s2)cc1</smiles>

Yellow crystal; mp 96-98 ${ }^{\circ} \mathrm{C}$ (not reported); ${ }^{1} \mathrm{H}$ NMR (300 MHz, $\left.\mathrm{CDCl}_{3}\right) \delta 8.47-8.50$ (m, 2H, ArH), 8.23-8.26 (m, 1H, ArH), 8.01-8.04 (m, 1H, ArH), 7.52-7.62 (m, 2H, $\mathrm{ArH}), 7.37$ (d, J 7.8 Hz, 2H, $\mathrm{ArH}), 2.47\left(\mathrm{~s}, 3 \mathrm{H}, \mathrm{CH}_{3}\right)$; ${ }^{13} \mathrm{C}$ NMR $\left(75 \mathrm{MHz}, \mathrm{CDCl}_{3}\right) \delta 184.8,167.4,153.8,145.0$, 136.9, 132.3, 131.4, 129.2, 127.4, 126.8, 125.6, 122.1, 21.8; MS (ESI): $m / z(\%) 254\left([\mathrm{M}+\mathrm{H}]^{+}, 100\right)$. 2-(4-chlorobenzoyl)benzothiazole $(\mathbf{4 c})^{12}$<smiles>O=C(c1ccc(Cl)cc1)c1nc2ccccc2s1</smiles>

White crystal, mp $100-102^{\circ} \mathrm{C}\left(102-103^{\circ} \mathrm{C}\right)^{12}$; ${ }^{1} \mathrm{H}$ NMR $\left(\mathrm{CDCl}_{3}, 300 \mathrm{MHz}\right) \delta 8.54-8.59$ (m, 2H, $\left.\mathrm{ArH}\right), 8.23-8.27$ (m, $1 \mathrm{H}, \mathrm{ArH}), 8.02-8.05$ (m, 1H, ArH), 7.53-7.64 (m, 4H, ArH); ${ }^{13} \mathrm{C} \mathrm{NMR}\left(75 \mathrm{MHz}, \mathrm{CDCl}_{3}\right) \delta 183.9,166.7,153.7,140.5$, 137.0, 133.1, 132.7, 128.8, 127.7, 127.0, 125.7, 122.1; MS (ESI): $m / z(\%) 274\left([\mathrm{M}+\mathrm{H}]^{+}, 100\right), 276\left([\mathrm{M}+2+\mathrm{H}]^{+}, 34\right)$.

2-(4-Bromobenzoyl)benzothiazole $(\mathbf{4 d})^{11}$<smiles>O=C(c1ccc(Br)cc1)c1nc2ccccc2s1</smiles>

Yellow crystal, mp $123-124{ }^{\circ} \mathrm{C}$ (not reported); ${ }^{1} \mathrm{H}$ NMR $\left(\mathrm{CDCl}_{3}, 300 \mathrm{MHz}\right) \delta 8.46-8.49(\mathrm{~m}, 2 \mathrm{H}, \mathrm{ArH}), 8.23-8.26$ (m, 1H, ArH), 8.02-8.07 (m, 1H, ArH), 7.70-7.73 (m, $2 \mathrm{H}, \mathrm{ArH}), 7.56-7.61(\mathrm{~m}, 2 \mathrm{H}, \mathrm{ArH}) ;{ }^{13} \mathrm{C}$ NMR $(75 \mathrm{MHz}$, $\left.\mathrm{CDCl}_{3}\right) \delta 184.1,166.7,153.8,137.0,133.6,132.7,131.8$, 129.5, 127.8, 127.0, 125.7, 122.2; MS (ESI): $\mathrm{m} / \mathrm{z}$ (\%) 317 $\left([\mathrm{M}+\mathrm{H}]^{+}, 100\right), 319\left([\mathrm{M}+2+\mathrm{H}]^{+}, 98\right)$.

\section{Reference}

1. Kodomari, M.; Tamaru, Y.; Aoyama, T.; Synth. Commun. 2004, 34, 3029.

2. Chowdhury, F. A.; Cole, E. R.; Crank, G.; J. Chromatogr. 1979, 170, 73.

3. Courtot, C.; Tchelitcheff, S.; Compt. Rend. 1943, 217, 231.

4. Deligeorgiev, T. G.; Dyes and Pigments 1990, 12, 243.

5. Paul, S.; Gupta, M.; Gupta, R.; Synth. Commun. 2002, 32, 3541.

6. Li, Y.; Wang, Y. L.; Wang, J. Y.; Chem. Lett. 2006, 35, 460.

7. Perry, R. J.; Wilson, B. D.; Organometallics 1994, 13, 3346.

8. Rostamizadeh, S.; Housaini, S. A. G.; Phosphorus, Sulfur Silicon Relat. Elem. 2005, 180, 1321.

9. Matsushita, H.; Lee, S. H.; Joung, M.; Clapham, B.; K. Janda, D.; Tetrahedron Lett. 2004, 45, 313.

10. Boga, C.; Stengel, R.; Abdayem, R.; Vecchio, E. D.; Forlani, L.; Todesco, P. E.; J. Org. Chem. 2004, 69, 8903.

11. Singh, H.; Singh, D. J.; Kumar, S.; Indian J. Chem., Sect. B: Org. Chem. Incl. Med. Chem. 1992, 31B, 217.

12. Caronna, T.; Galli, R.; Malatesta, V.; J. Chem. Soc. C 1971, 1747. 

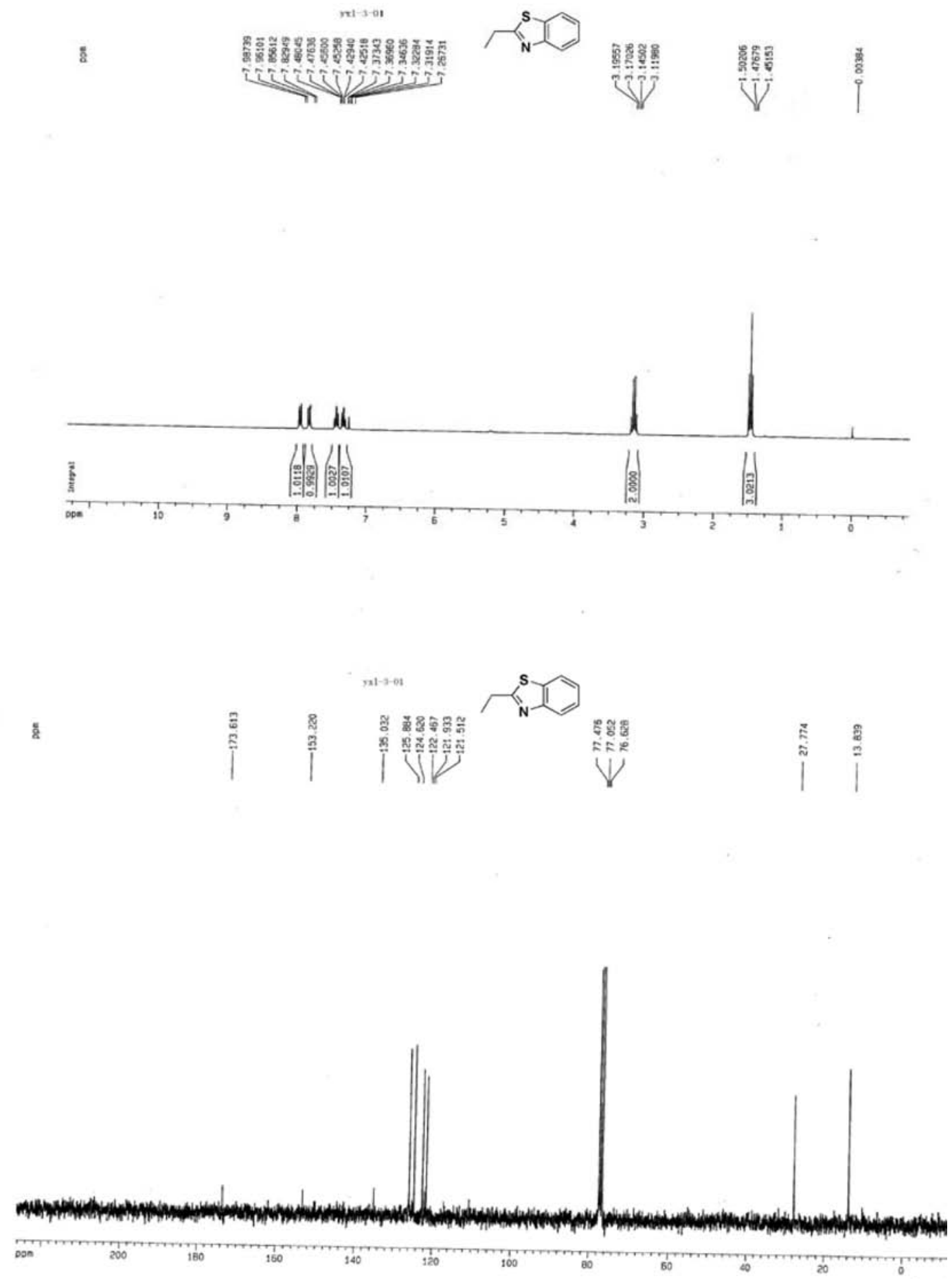

Figure S1. ${ }^{1} \mathrm{H} \mathrm{NMR}$ of $\mathbf{3 a}\left(300 \mathrm{MHz}, \mathrm{CDCl}_{3}\right)$ and ${ }^{13} \mathrm{C} \mathrm{NMR}$ of $\mathbf{3 a}\left(75 \mathrm{MHz}, \mathrm{CDCl}_{3}\right)$. 

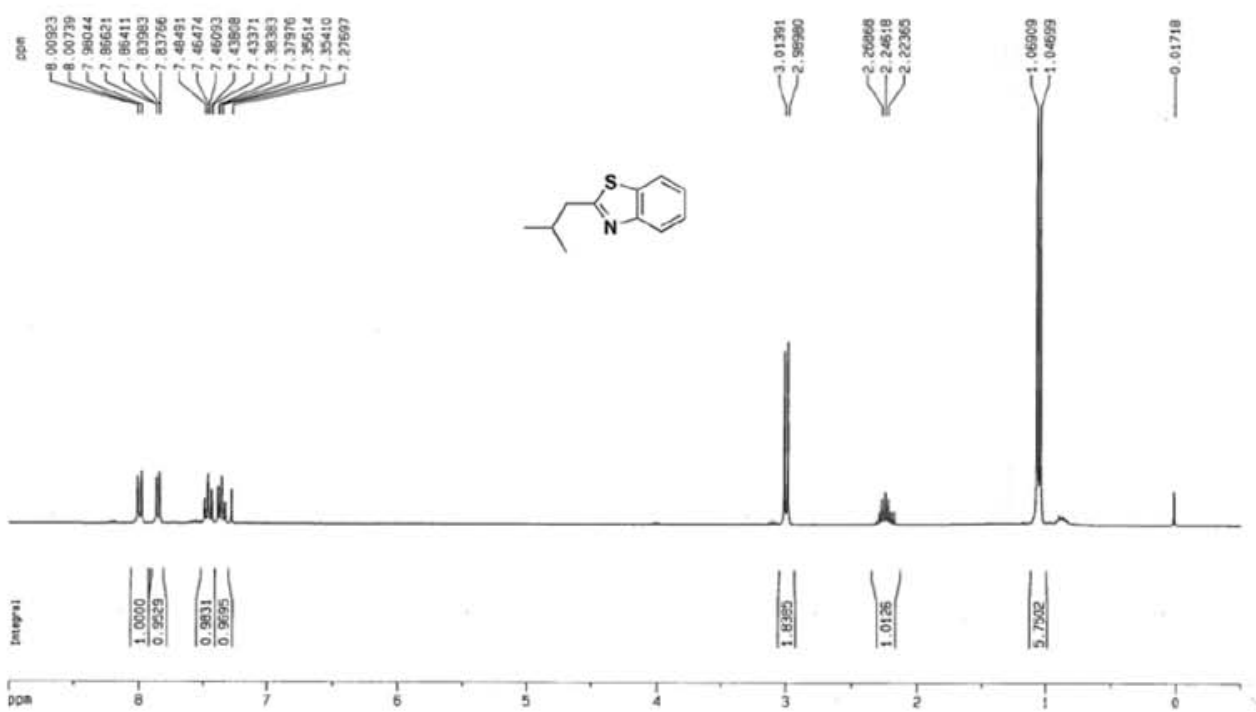

$x \mathbf{1 - 3 - 2}$

哀
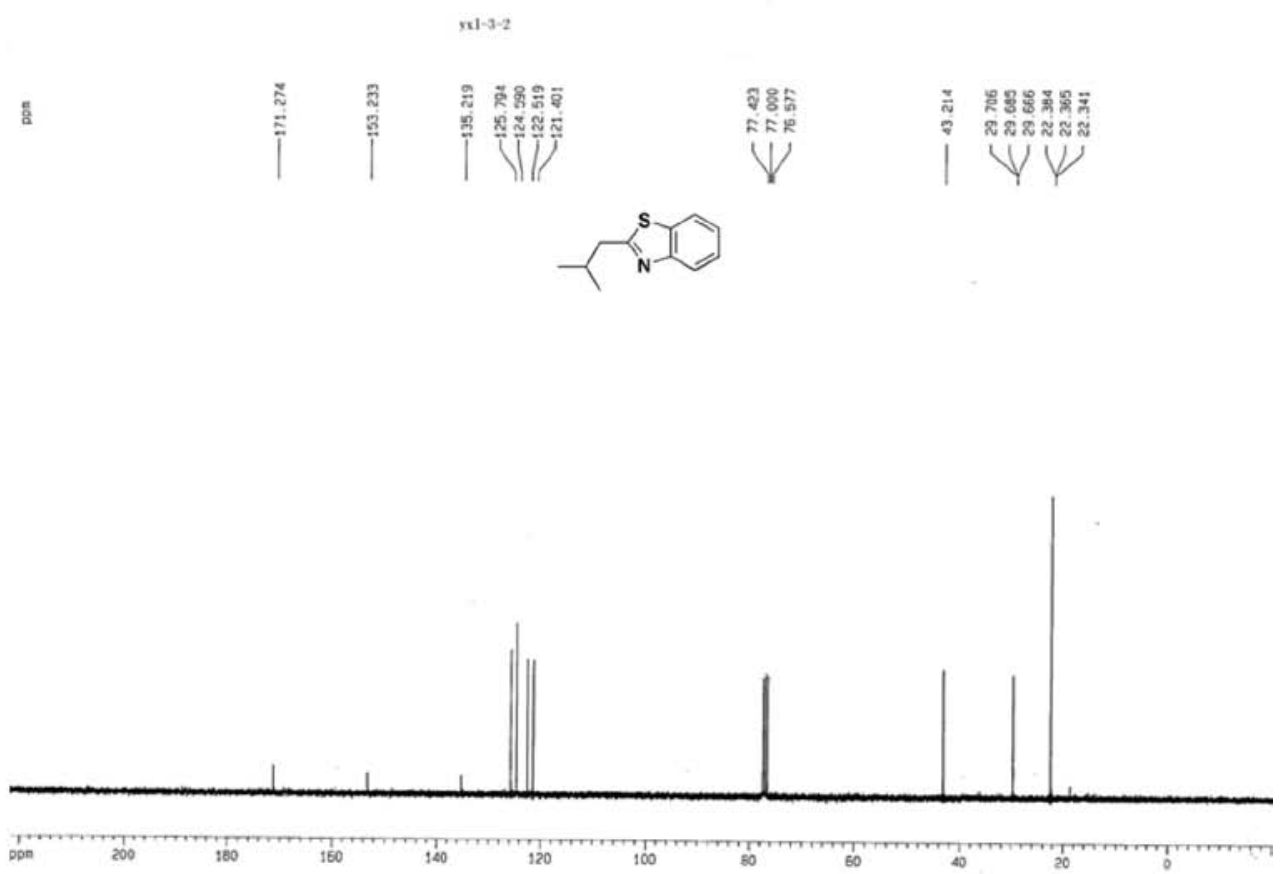

Figure S2. ${ }^{1} \mathrm{H}$ NMR of $\mathbf{3 b}\left(300 \mathrm{MHz}, \mathrm{CDCl}_{3}\right)$ and ${ }^{13} \mathrm{C} \mathrm{NMR}$ of $\mathbf{3 b}\left(75 \mathrm{MHz}, \mathrm{CDCl}_{3}\right)$. 

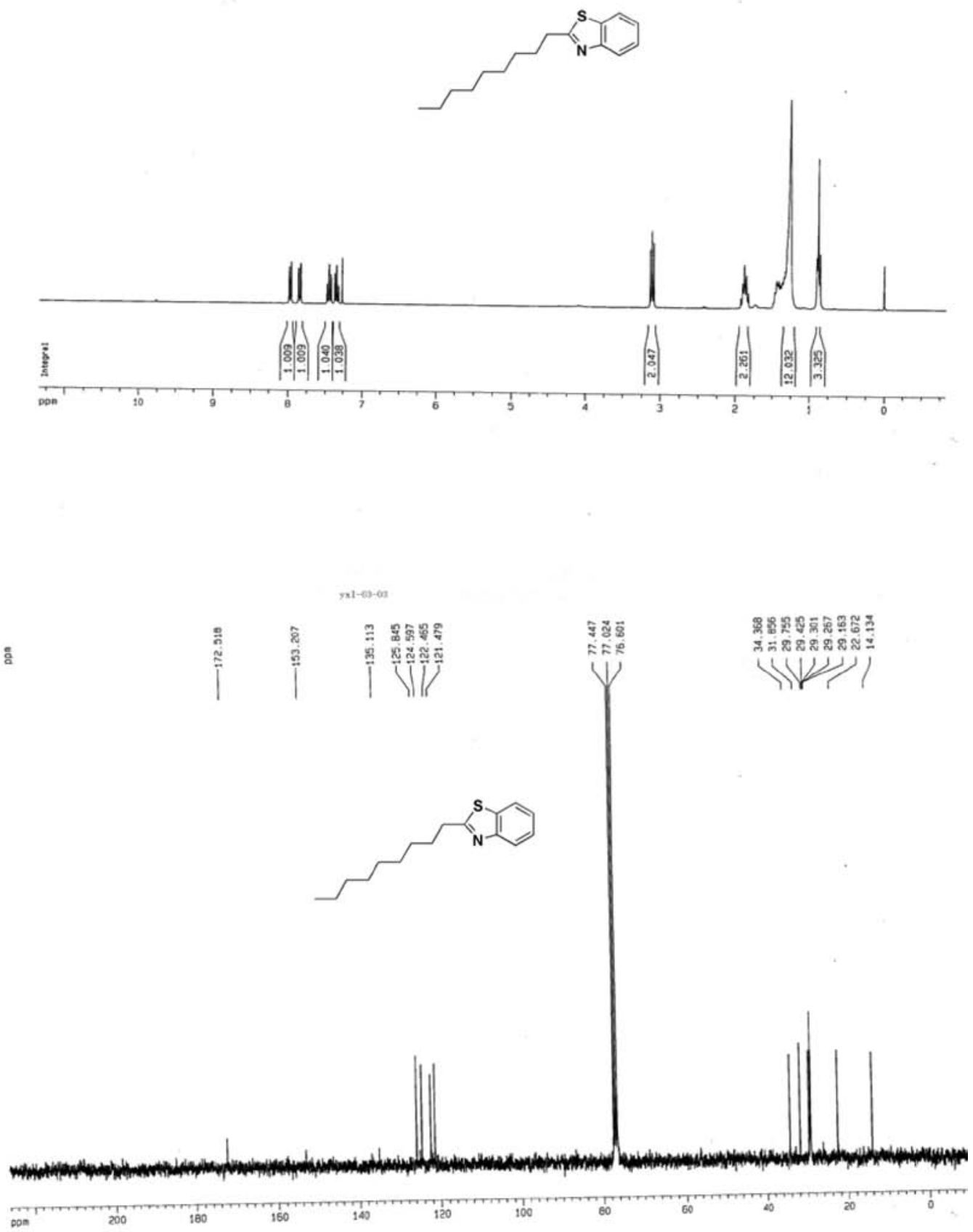

Figure S3. ${ }^{1} \mathrm{H}$ NMR of $\mathbf{3 c}\left(300 \mathrm{MHz}, \mathrm{CDCl}_{3}\right)$ and ${ }^{13} \mathrm{C} \mathrm{NMR}$ of $\mathbf{3 c}\left(75 \mathrm{MHz}, \mathrm{CDCl}_{3}\right)$. 
; $21-6-0 \mid-1$

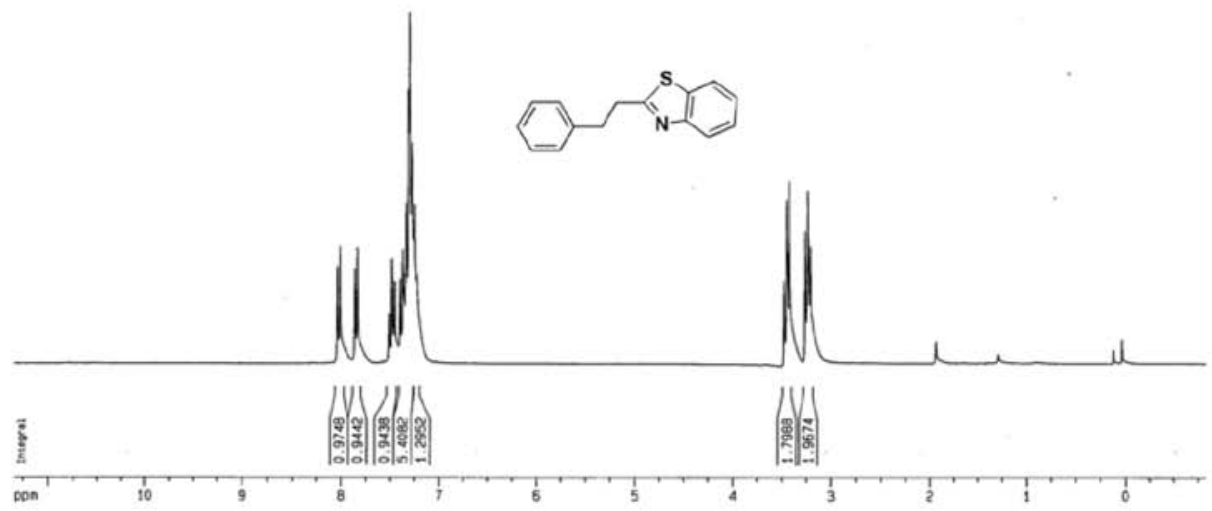

at - $05-61$

s
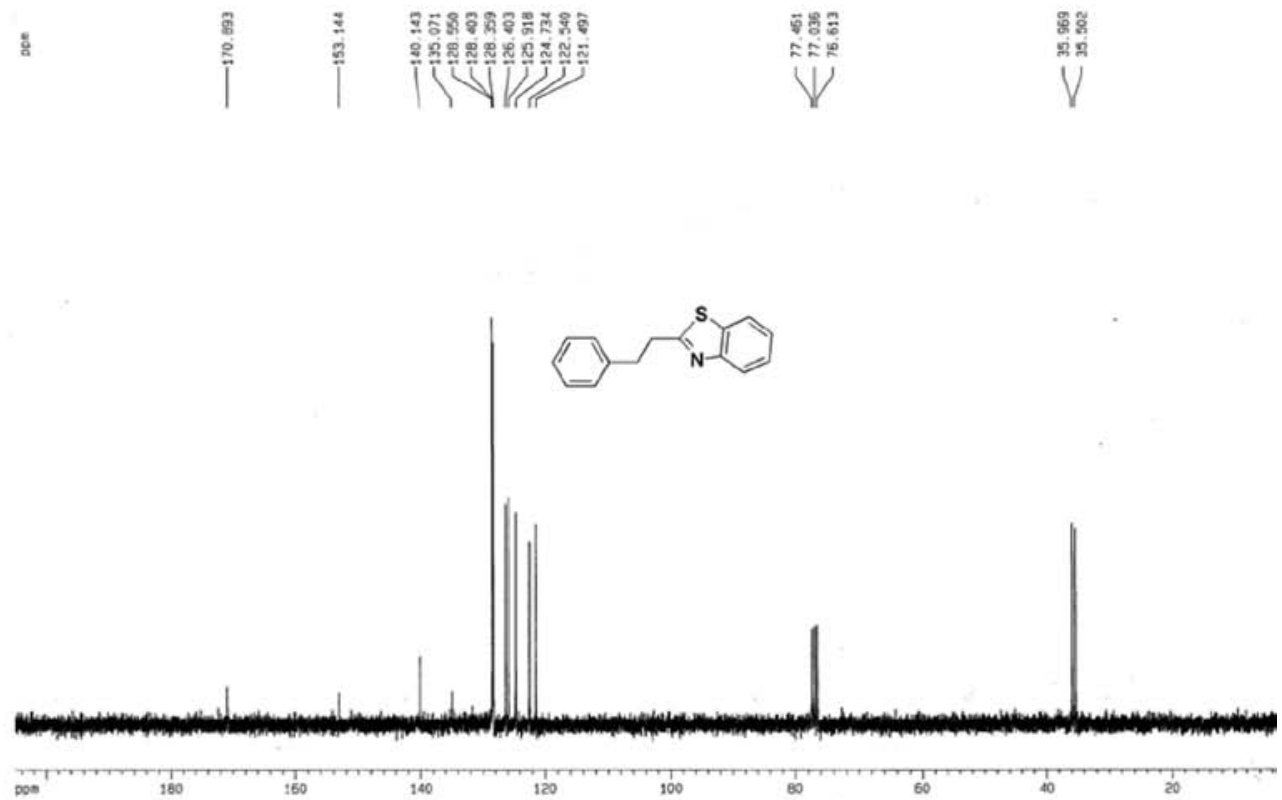

Figure S4. ${ }^{1} \mathrm{H}$ NMR of $\mathbf{3 d}\left(300 \mathrm{MHz}, \mathrm{CDCl}_{3}\right)$ and ${ }^{13} \mathrm{C}$ NMR of $\mathbf{3 d}\left(75 \mathrm{MHz}, \mathrm{CDCl}_{3}\right)$. 


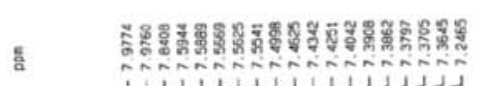
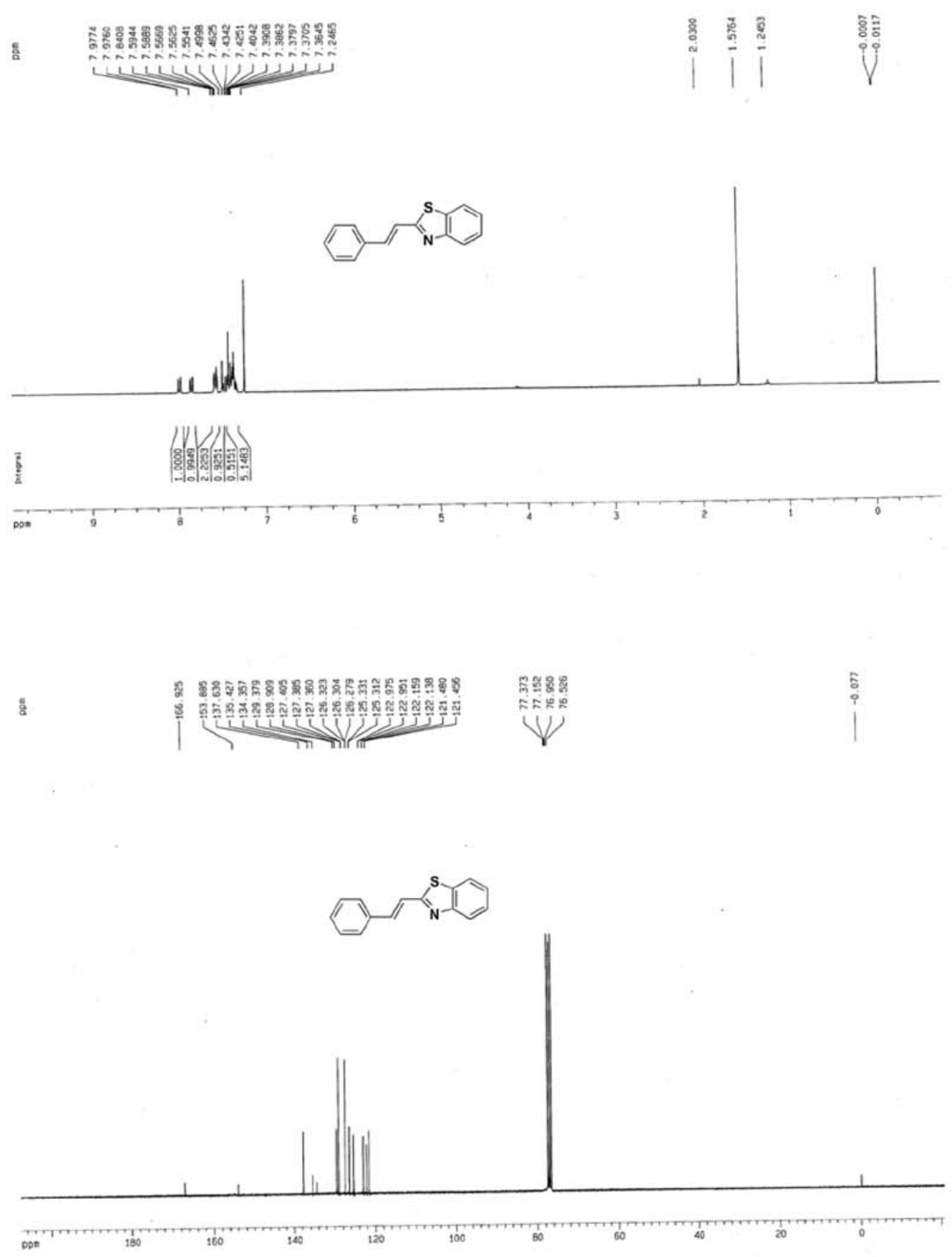

Figure S5. ${ }^{1} \mathrm{H} \mathrm{NMR}$ of $\mathbf{3 e}\left(300 \mathrm{MHz}, \mathrm{CDCl}_{3}\right)$ and ${ }^{13} \mathrm{C} \mathrm{NMR}$ of $\mathbf{3 e}\left(75 \mathrm{MHz}, \mathrm{CDCl}_{3}\right)$. 

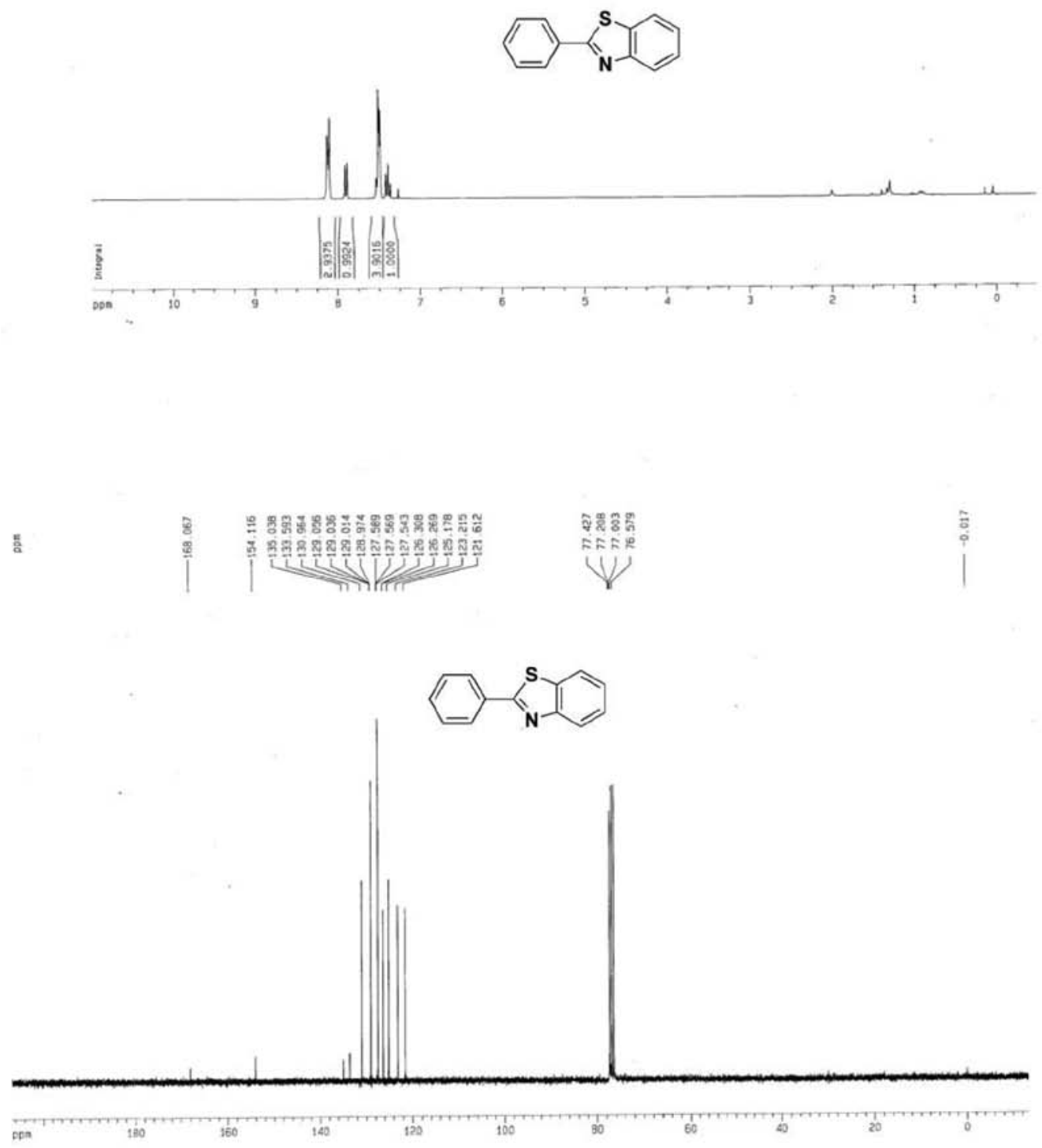

Figure S6. ${ }^{1} \mathrm{H}$ NMR of $\mathbf{3 f}\left(300 \mathrm{MHz}, \mathrm{CDCl}_{3}\right)$ and ${ }^{13} \mathrm{C} \mathrm{NMR}$ of $\mathbf{3 f}\left(75 \mathrm{MHz}, \mathrm{CDCl}_{3}\right)$. 


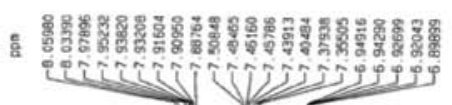

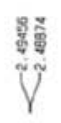
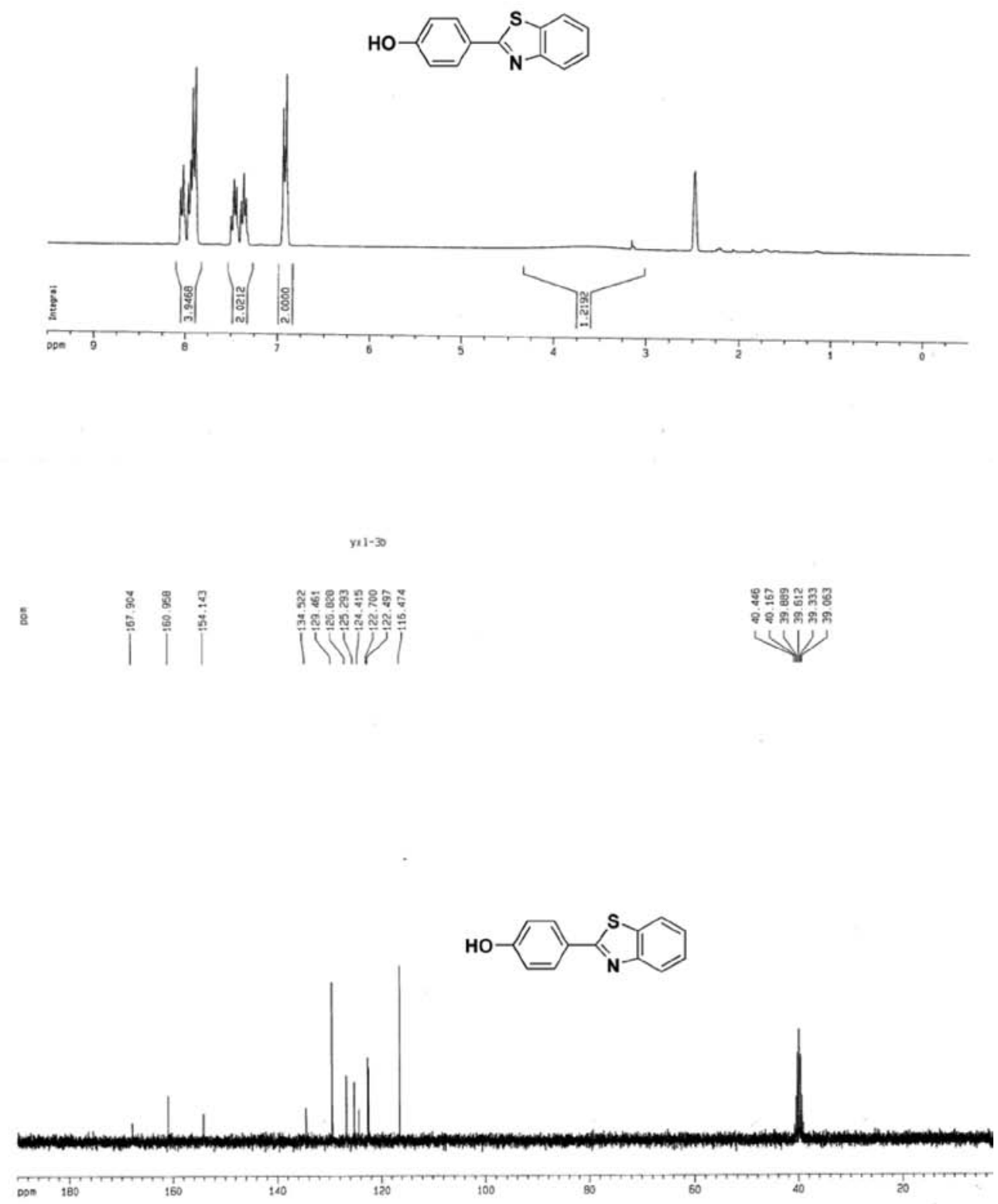

Figure S7. ${ }^{1} \mathrm{H} \mathrm{NMR}$ of $\mathbf{3 g}\left(300 \mathrm{MHz}, \mathrm{CDCl}_{3}\right)$ and ${ }^{13} \mathrm{C} \mathrm{NMR}$ of $\mathbf{3 g}\left(75 \mathrm{MHz}, \mathrm{CDCl}_{3}\right)$. 


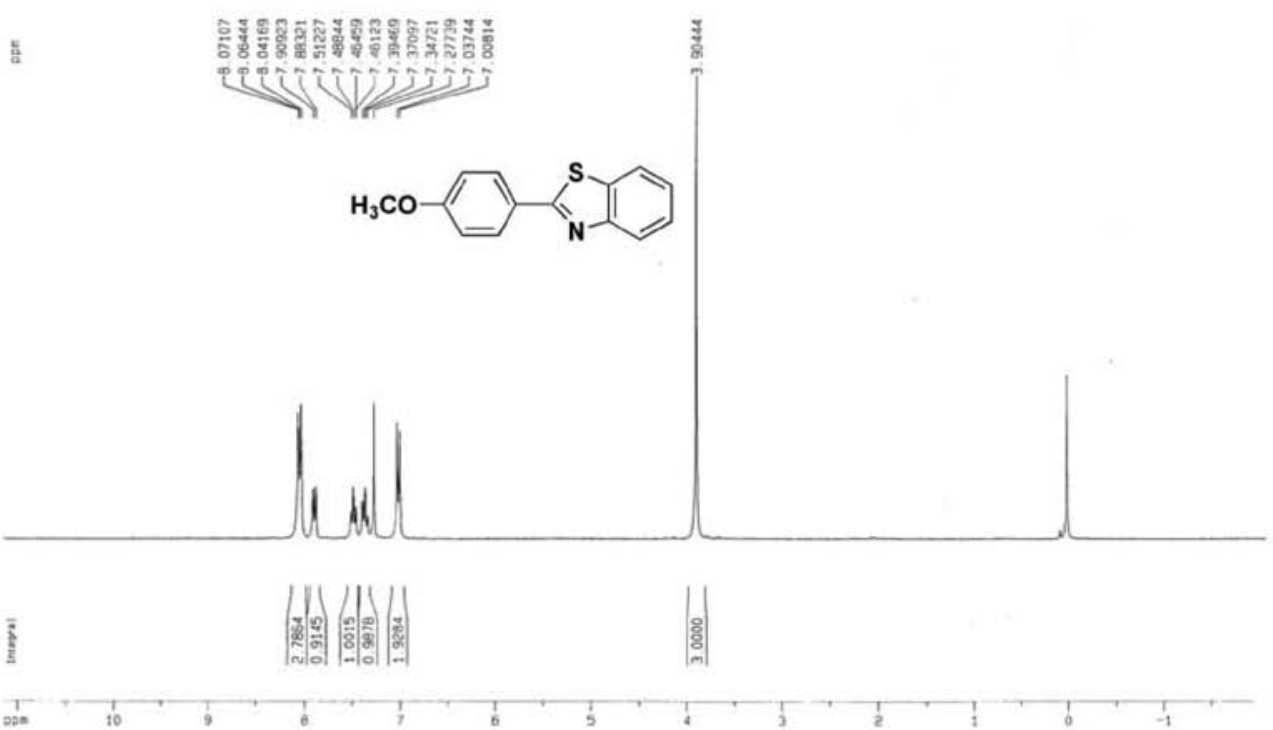

s

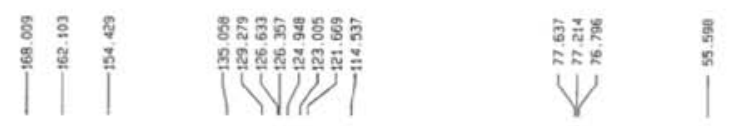
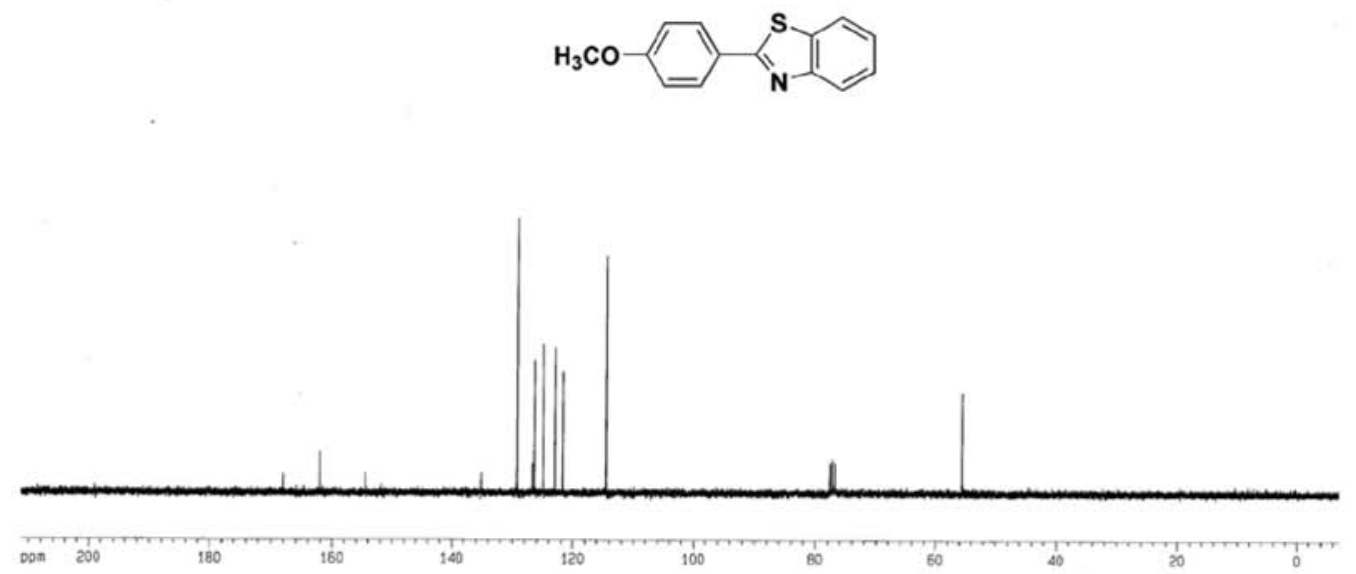

Figure S8. ${ }^{1} \mathrm{H}$ NMR of $\mathbf{3 h}\left(300 \mathrm{MHz}, \mathrm{CDCl}_{3}\right)$ and ${ }^{13} \mathrm{C} \mathrm{NMR}$ of $\mathbf{3 h}\left(75 \mathrm{MHz}, \mathrm{CDCl}_{3}\right)$. 


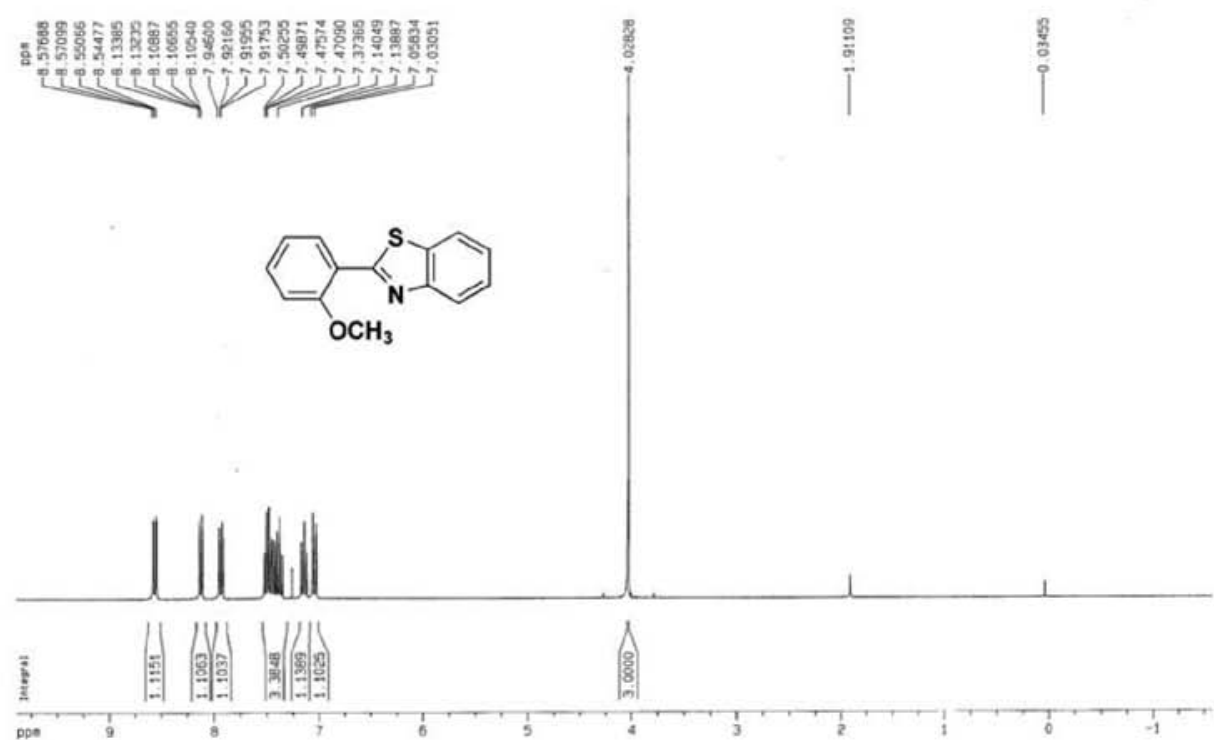

s
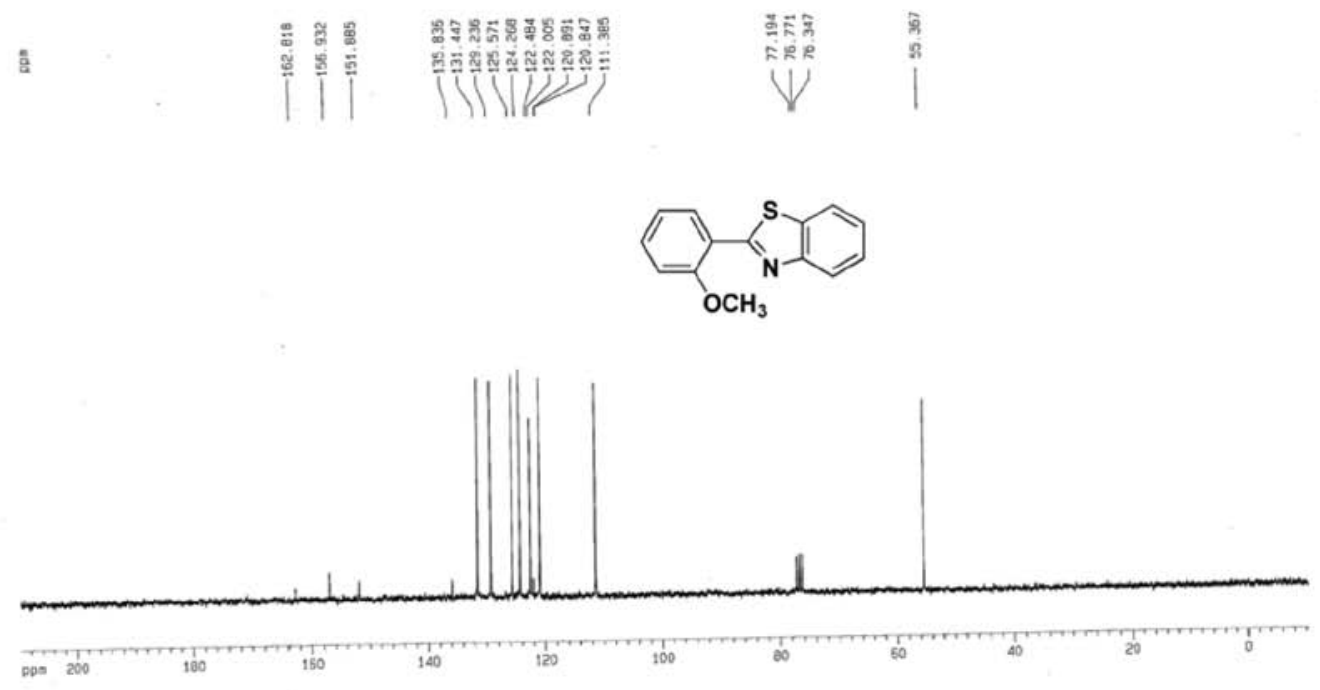

Figure S9. ${ }^{1} \mathrm{H} \mathrm{NMR}$ of $3 \mathbf{i}\left(300 \mathrm{MHz}, \mathrm{CDCl}_{3}\right)$ and ${ }^{13} \mathrm{C} \mathrm{NMR}$ of $3 \mathbf{i}\left(75 \mathrm{MHz}, \mathrm{CDCl}_{3}\right)$. 


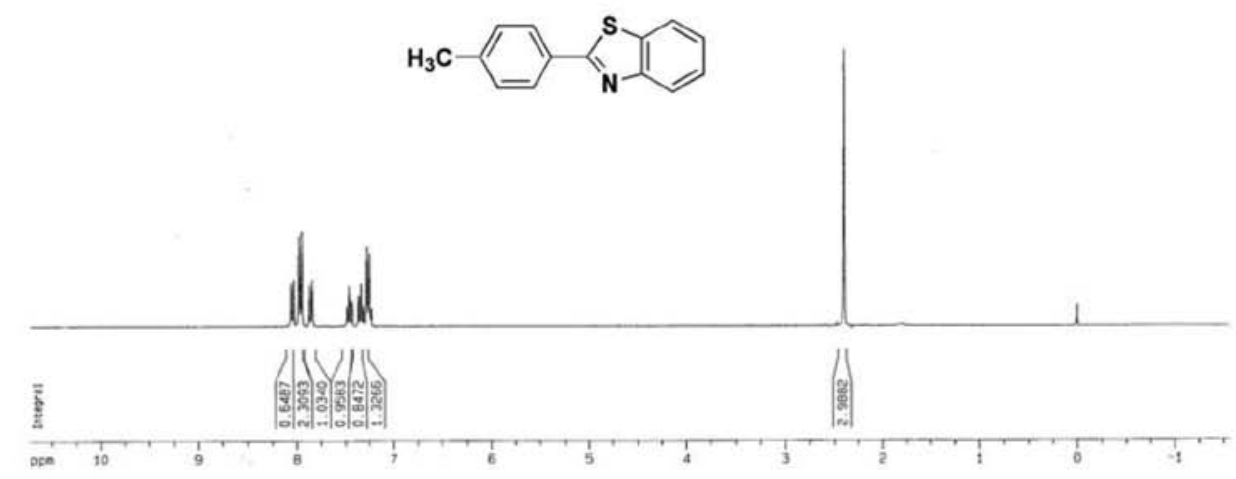

s
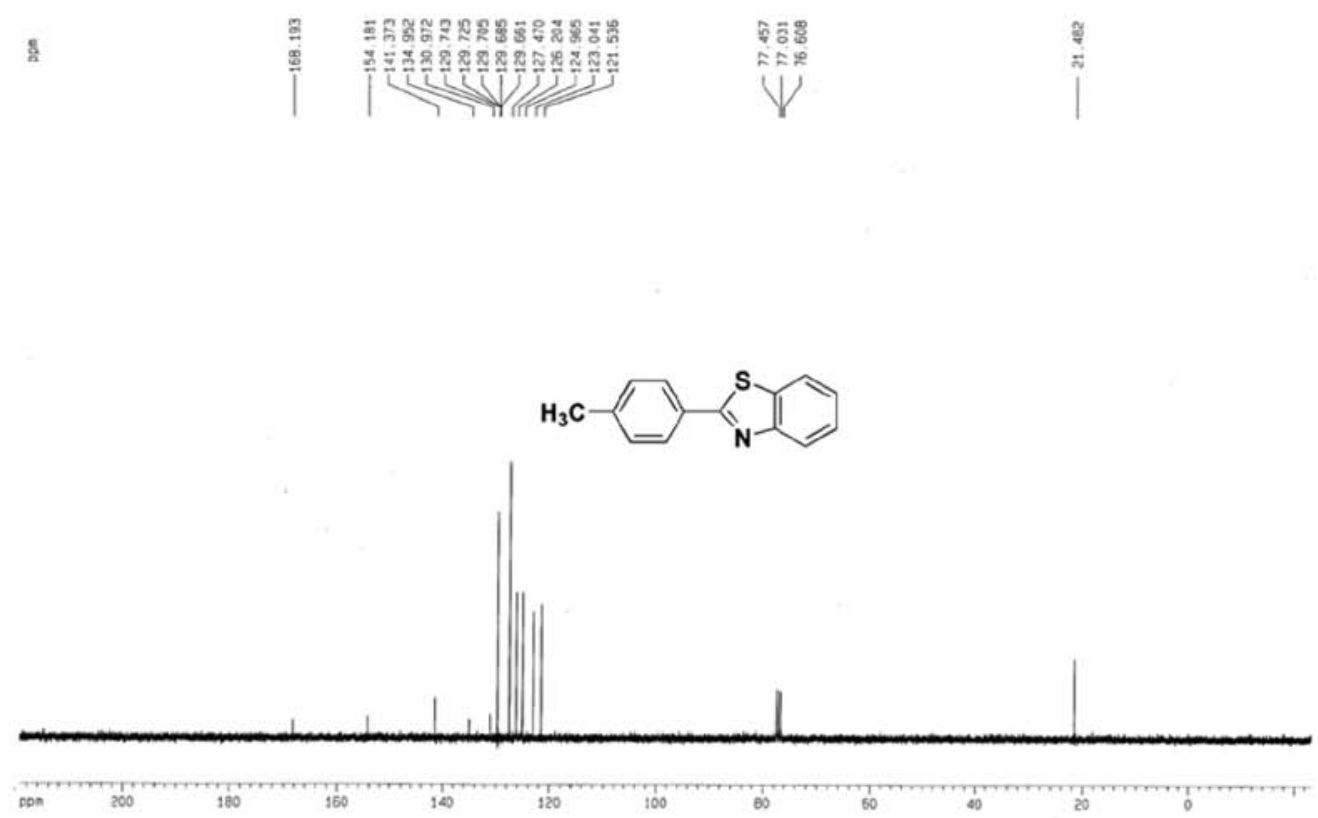

Figure S10. ${ }^{1} \mathrm{H}$ NMR of $\mathbf{3 j}$ ( $300 \mathrm{MHz}, \mathrm{CDCl}_{3}$ ) and ${ }^{13} \mathrm{C} \mathrm{NMR}$ of $\mathbf{3 j}\left(75 \mathrm{MHz}, \mathrm{CDCl}_{3}\right)$. 


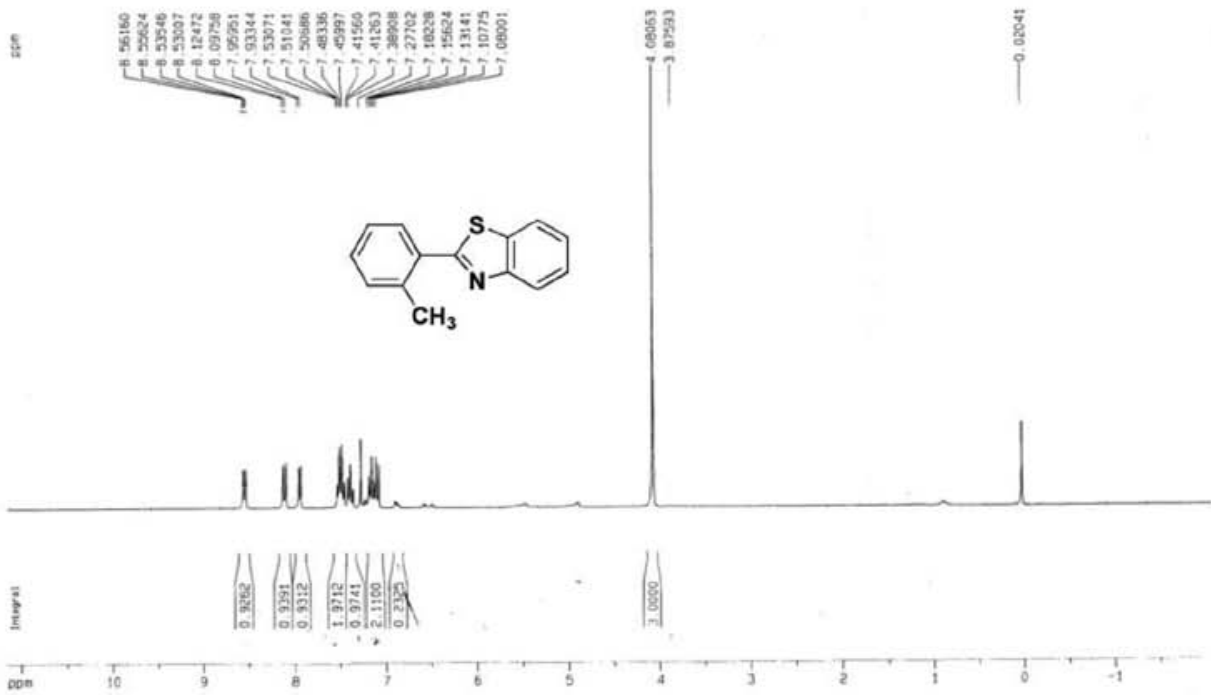

$y \times 1-03$.

a
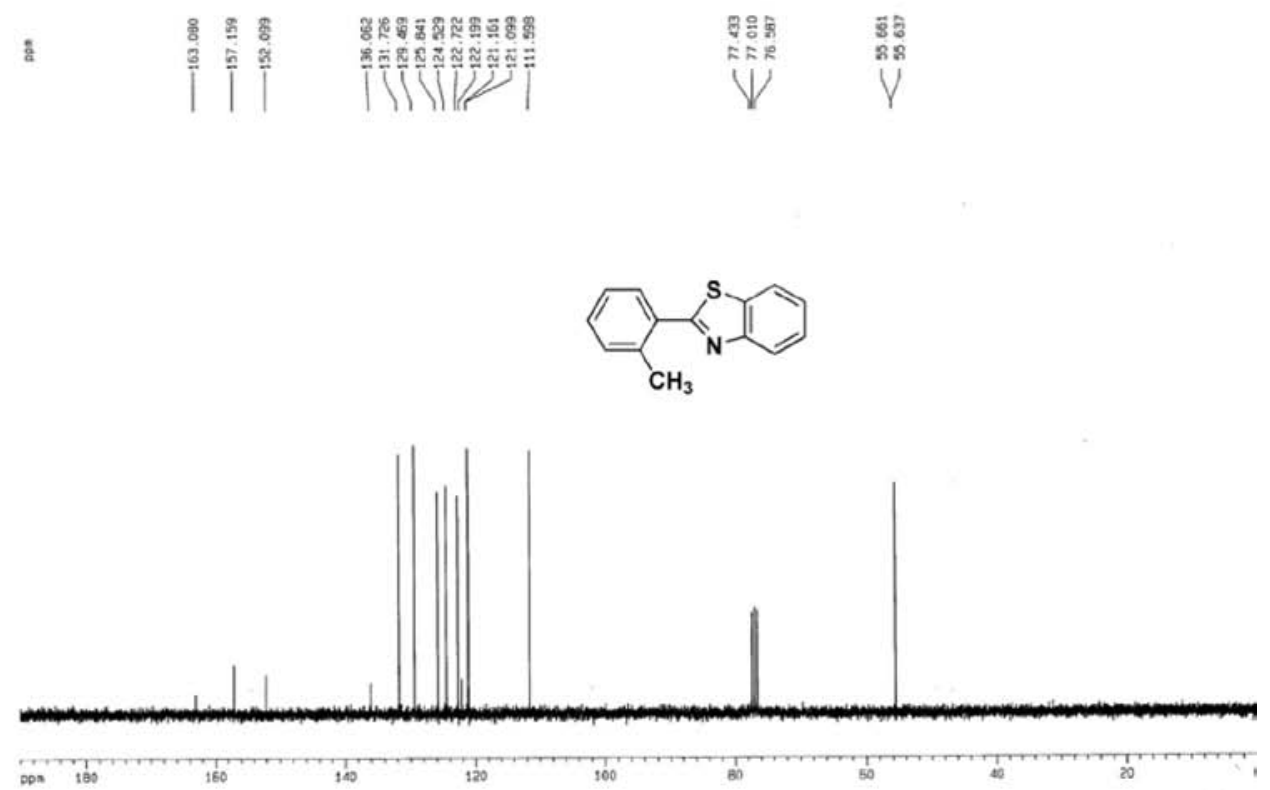

Figure S11. ${ }^{1} \mathrm{H}$ NMR of 3k $\left(300 \mathrm{MHz}, \mathrm{CDCl}_{3}\right)$ and ${ }^{13} \mathrm{C} \mathrm{NMR}$ of $\mathbf{3 k}\left(75 \mathrm{MHz}, \mathrm{CDCl}_{3}\right)$. 

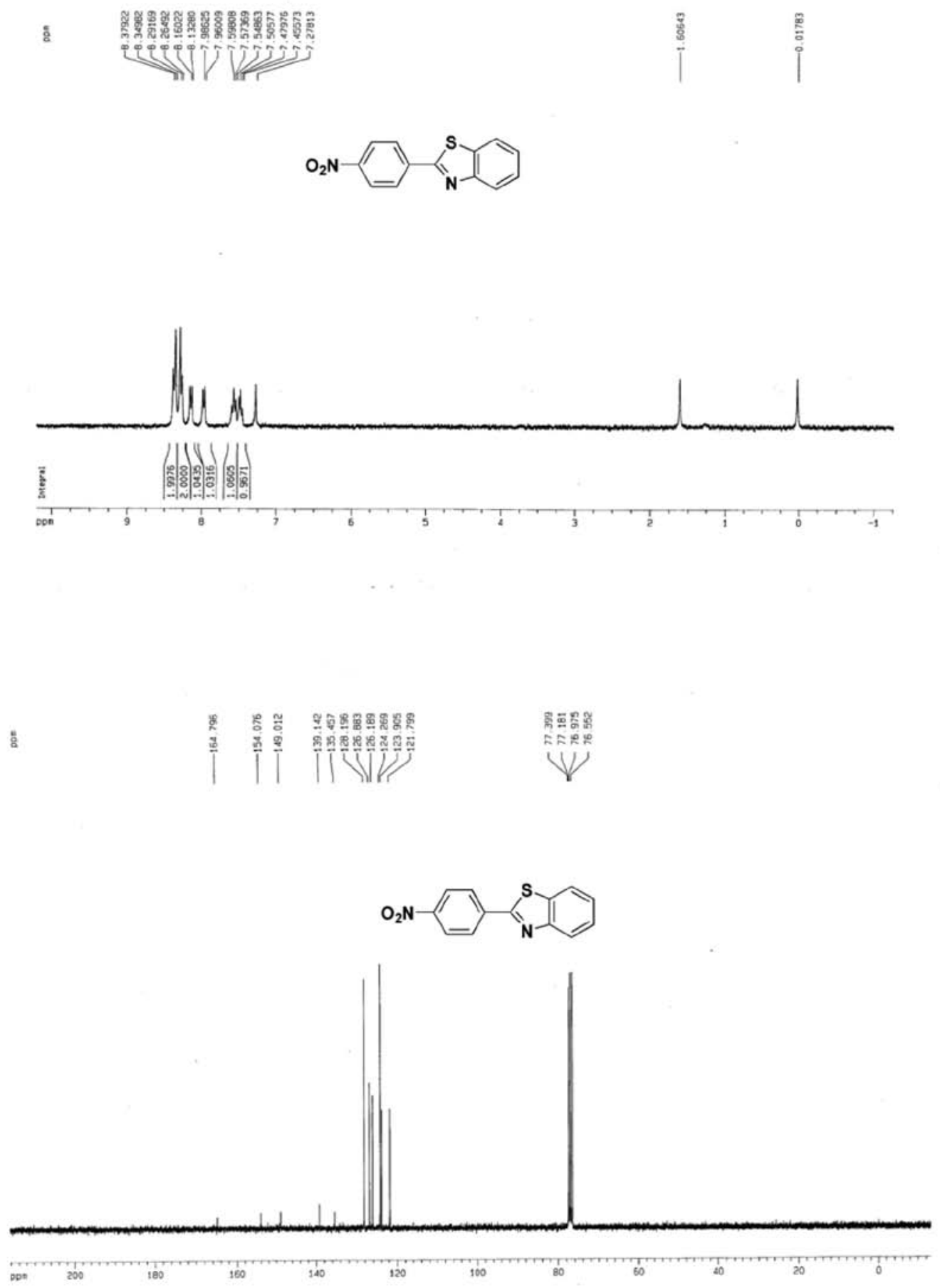

Figure S12. ${ }^{1} \mathrm{H}$ NMR of $31\left(300 \mathrm{MHz}, \mathrm{CDCl}_{3}\right)$ and ${ }^{13} \mathrm{C} \mathrm{NMR}$ of $3 \mathrm{l}\left(75 \mathrm{MHz}, \mathrm{CDCl}_{3}\right)$, 

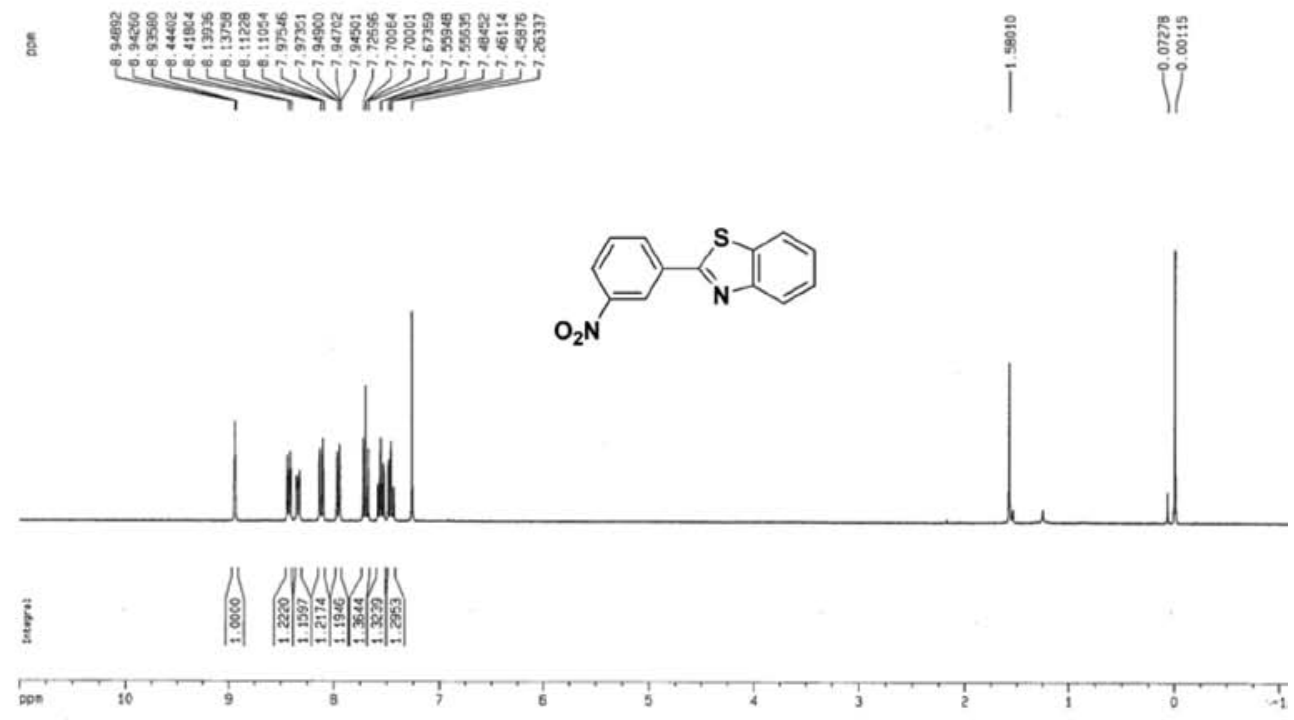

พxi-3h

ฐ

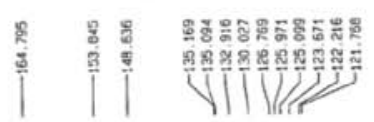

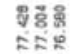

พิ

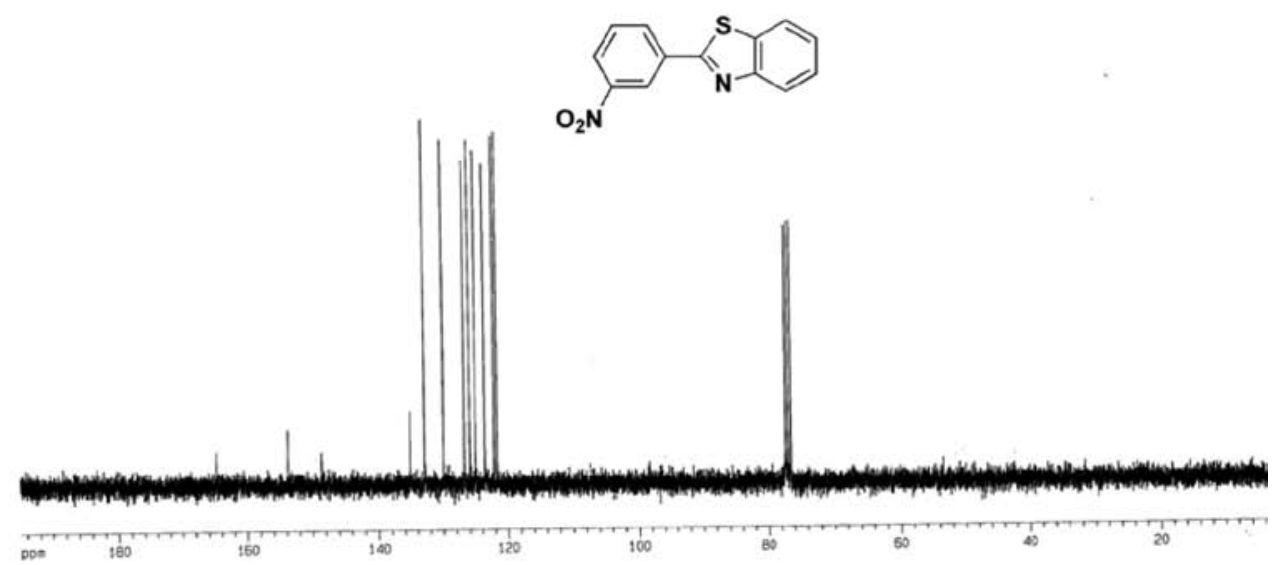

Figure S13. ${ }^{1} \mathrm{H}$ NMR of $\mathbf{3 m}\left(300 \mathrm{MHz}, \mathrm{CDCl}_{3}\right)$ and ${ }^{13} \mathrm{C} \mathrm{NMR}$ of $\mathbf{3 m}\left(75 \mathrm{MHz}, \mathrm{CDCl}_{3}\right)$. 


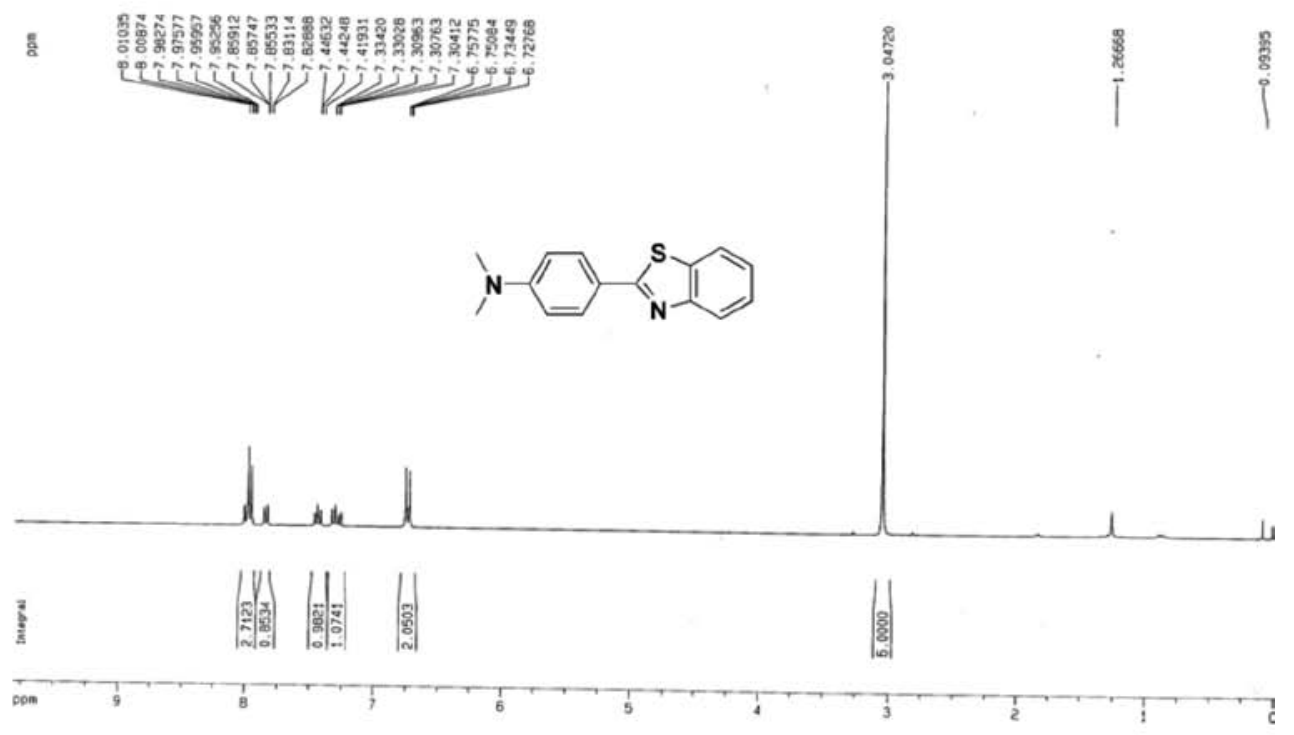

$\mid x \times 1-31$

5 $\quad$ |
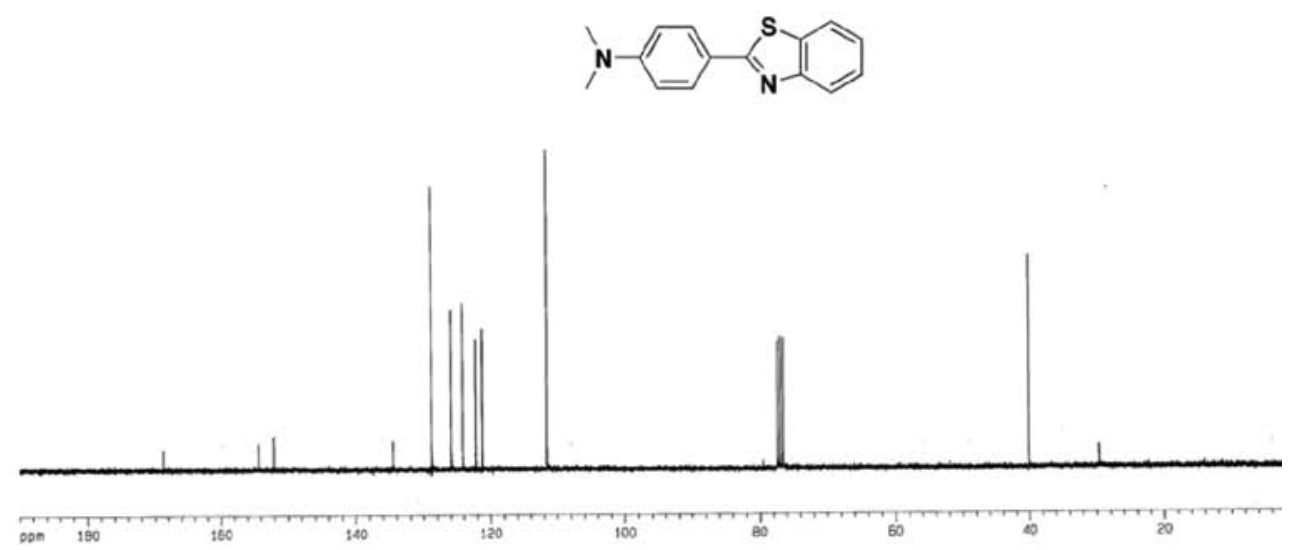

Figure S14. ${ }^{1} \mathrm{H}$ NMR of 3n (300 MHz, $\left.\mathrm{CDCl}_{3}\right)$ and ${ }^{13} \mathrm{C}$ NMR of $3 \mathbf{n}\left(75 \mathrm{MHz}, \mathrm{CDCl}_{3}\right)$. 
s

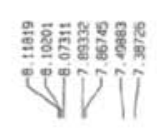

$\prod^{\stackrel{5}{\mathrm{j}}}$
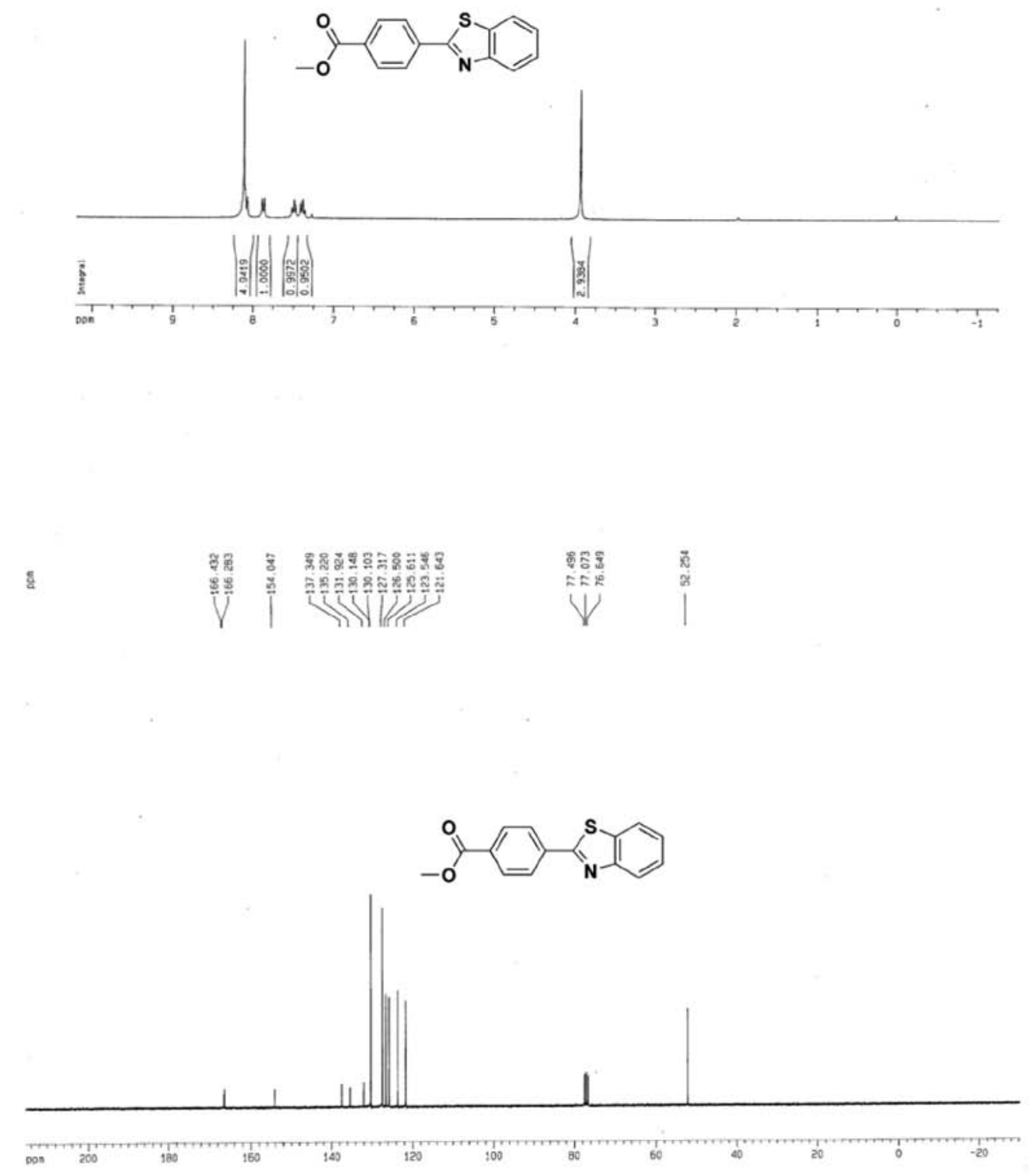

Figure S15. ${ }^{1} \mathrm{H}$ NMR of $\mathbf{3 o}\left(300 \mathrm{MHz}, \mathrm{CDCl}_{3}\right)$ and ${ }^{13} \mathrm{C} \mathrm{NMR}$ of $\mathbf{3 o}\left(75 \mathrm{MHz}, \mathrm{CDCl}_{3}\right)$. 

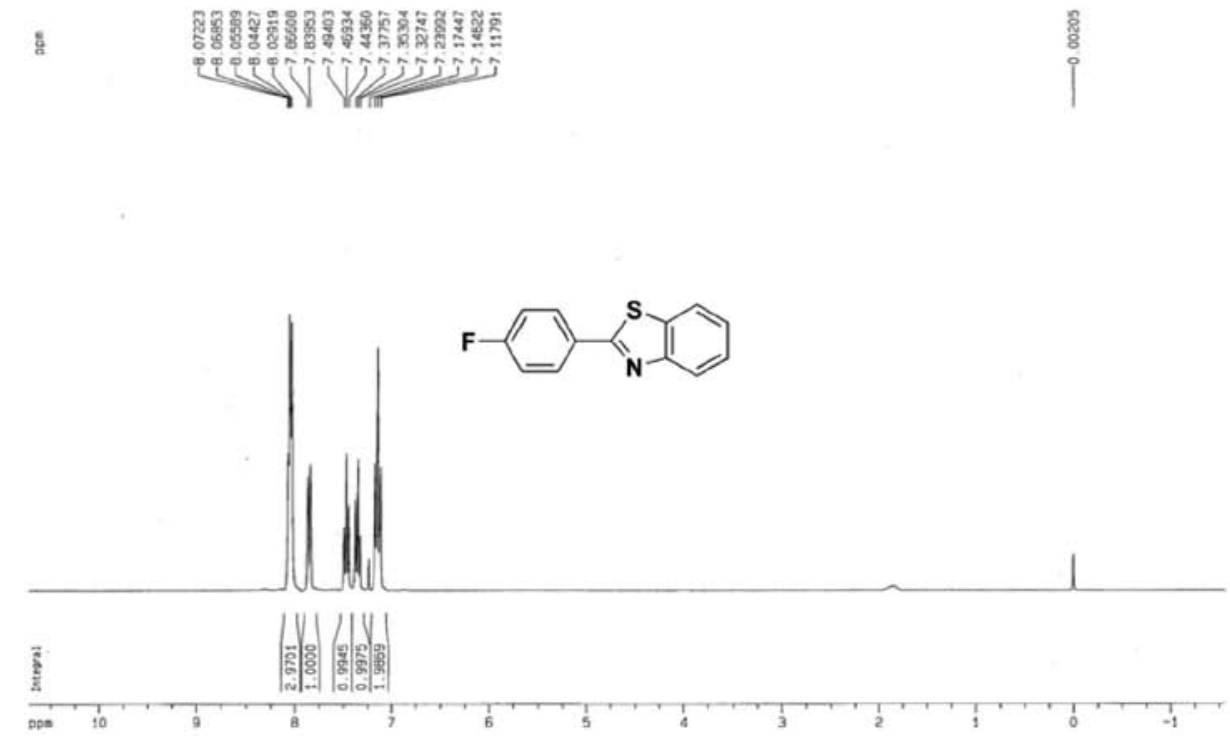

\&
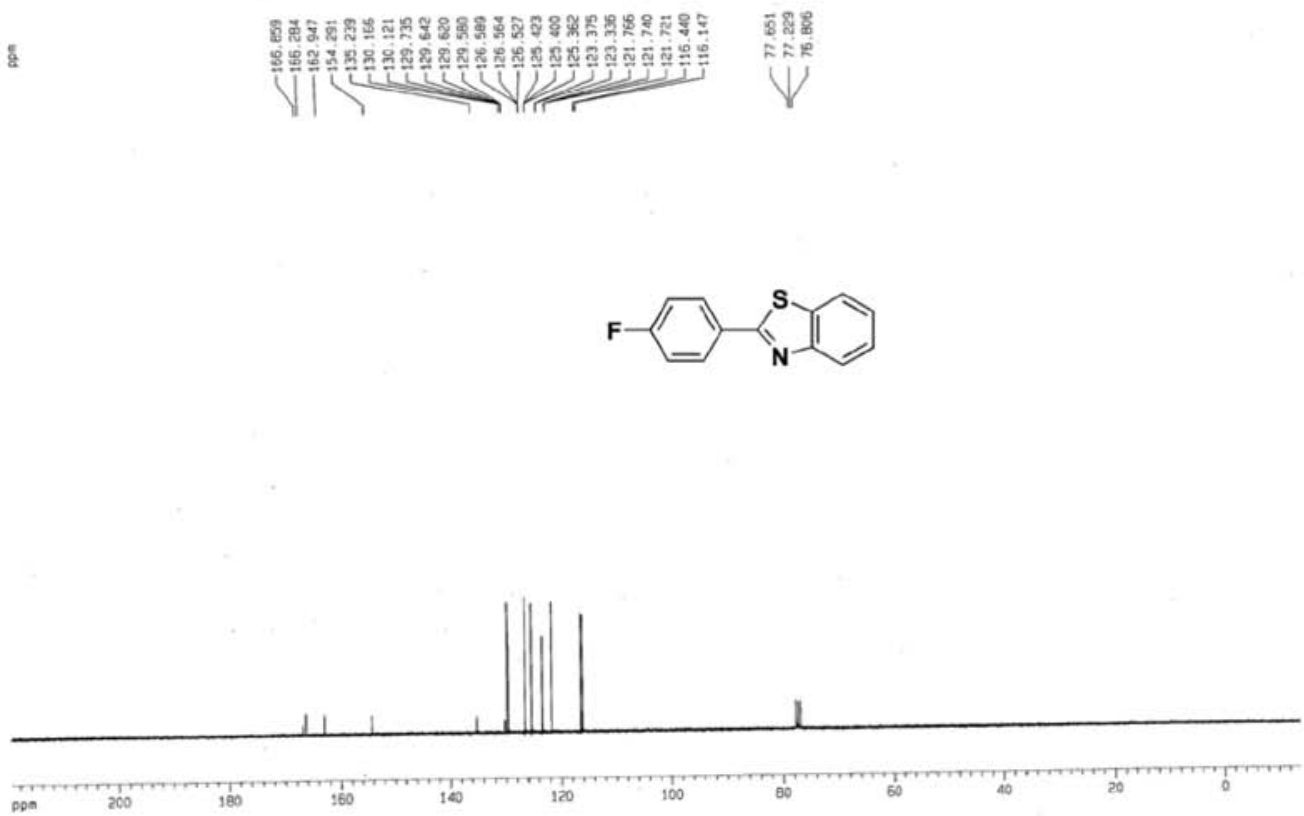

Figure S16. ${ }^{1} \mathrm{H}$ NMR of $\mathbf{3 p}\left(300 \mathrm{MHz}, \mathrm{CDCl}_{3}\right)$ and ${ }^{13} \mathrm{C} \mathrm{NMR}$ of $\mathbf{3 p}\left(75 \mathrm{MHz}, \mathrm{CDCl}_{3}\right)$. 
s

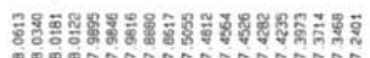

\begin{tabular}{l} 
: \\
i \\
\hline
\end{tabular}

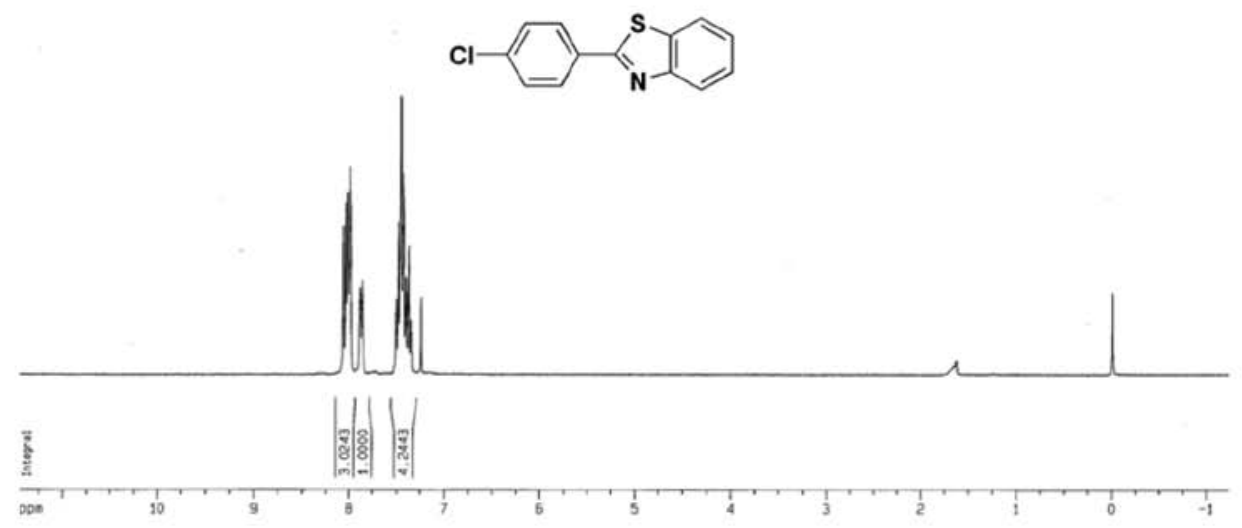

站
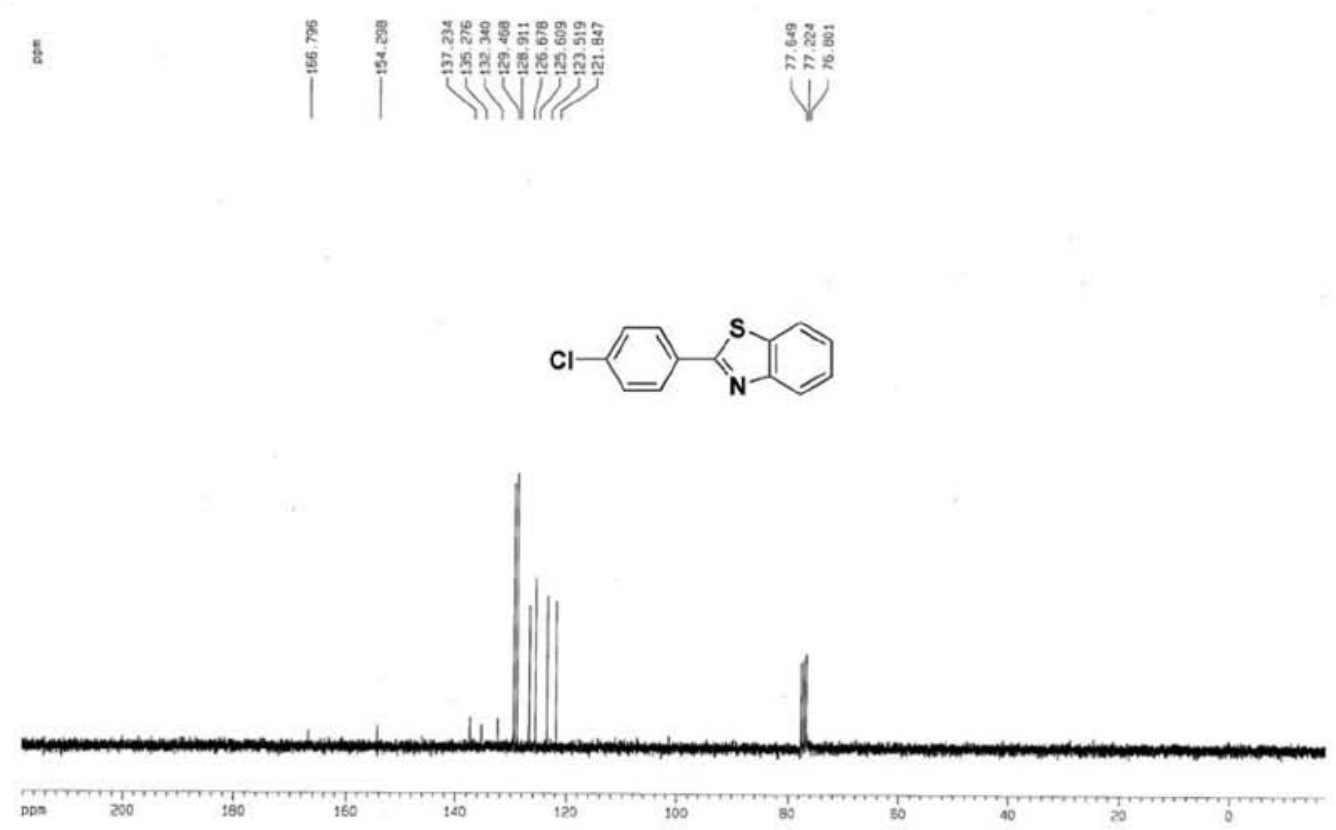

Figure S17. ${ }^{1} \mathrm{H}$ NMR of $\mathbf{3 q}\left(300 \mathrm{MHz}, \mathrm{CDCl}_{3}\right)$ and ${ }^{13} \mathrm{C} \mathrm{NMR}$ of $\mathbf{3 q}\left(75 \mathrm{MHz}, \mathrm{CDCl}_{3}\right)$. 


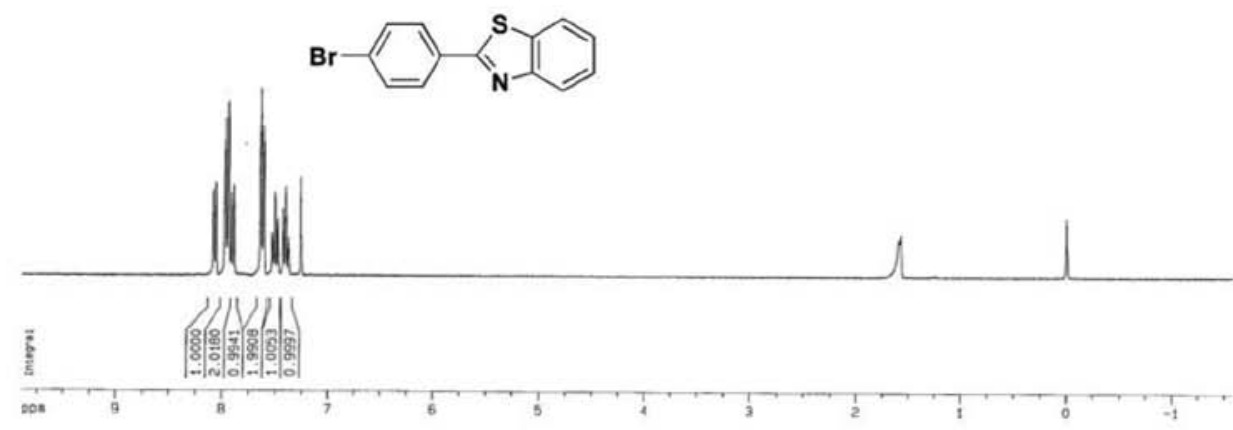

s
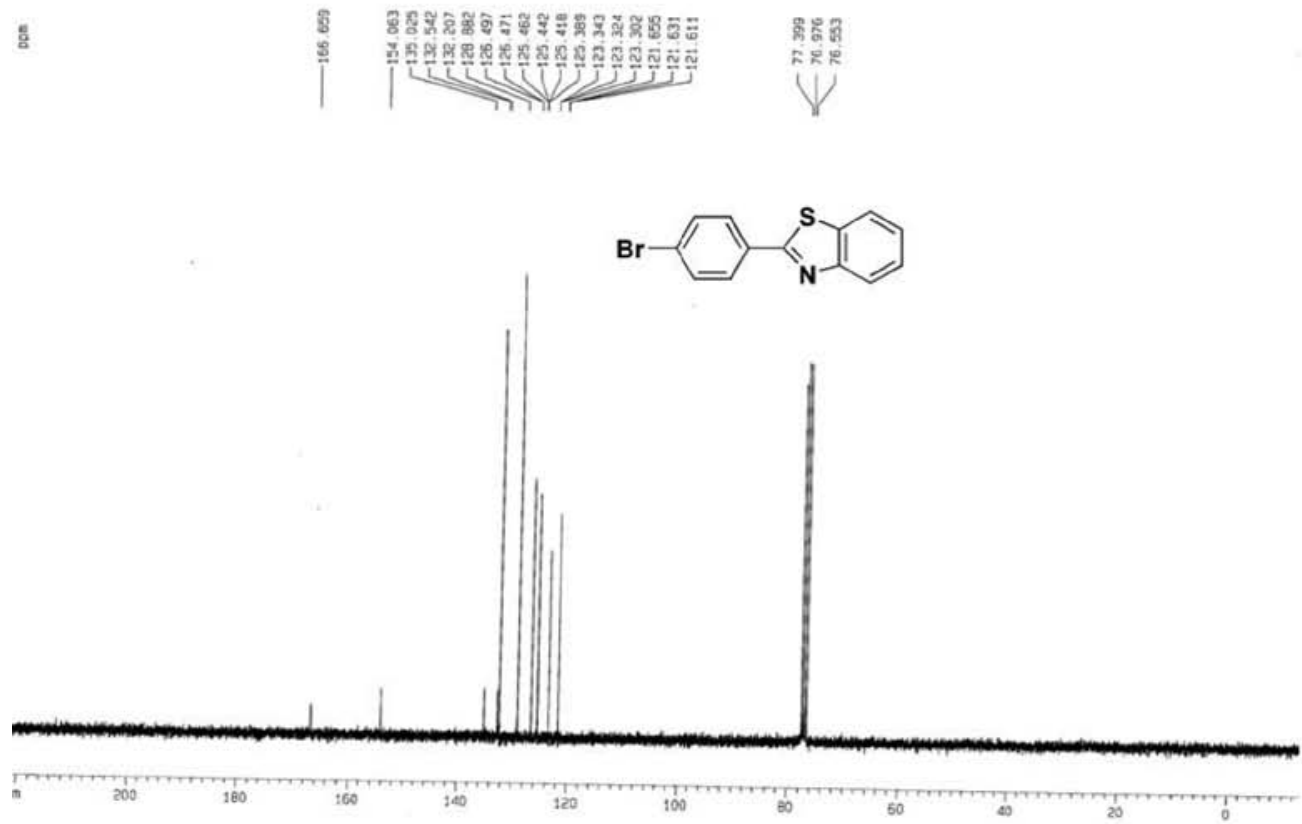

Figure S18. ${ }^{1} \mathrm{H}$ NMR of $\mathbf{3 r}\left(300 \mathrm{MHz}, \mathrm{CDCl}_{3}\right)$ and ${ }^{13} \mathrm{C} \mathrm{NMR}$ of $3 \mathbf{r}\left(75 \mathrm{MHz}, \mathrm{CDCl}_{3}\right)$. 

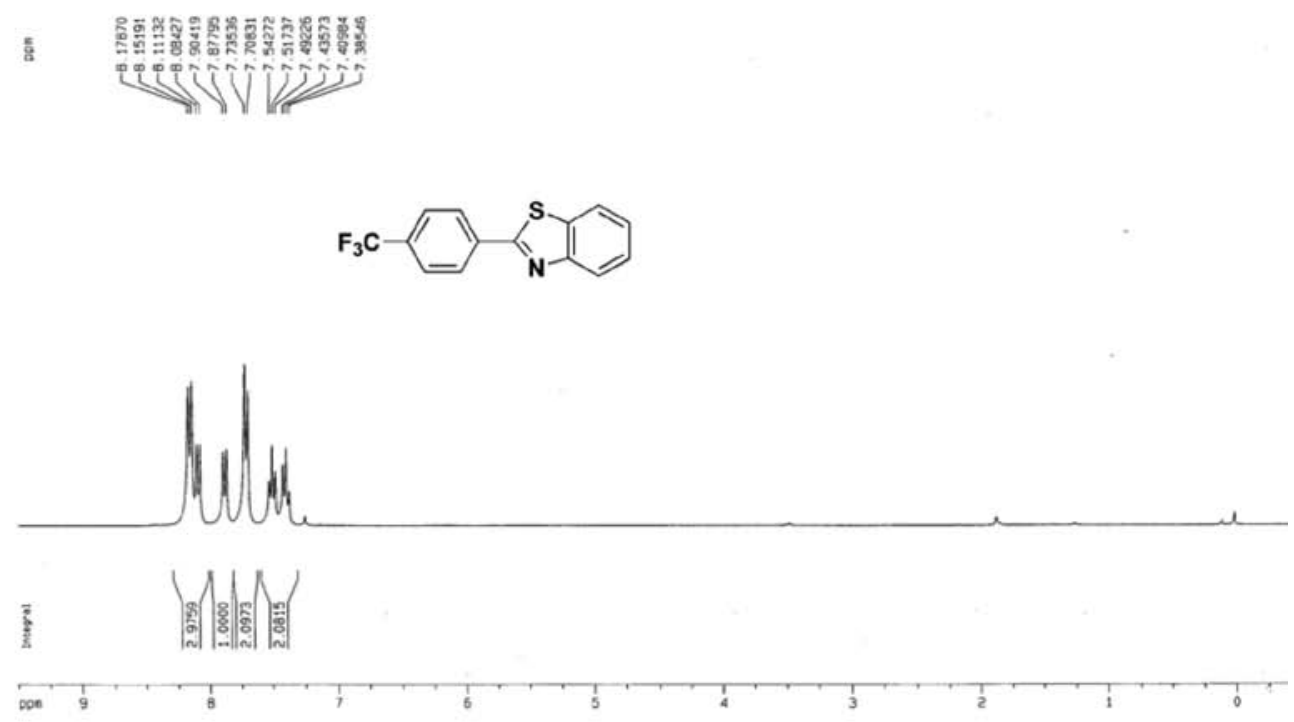

$y \times 1-3 n$
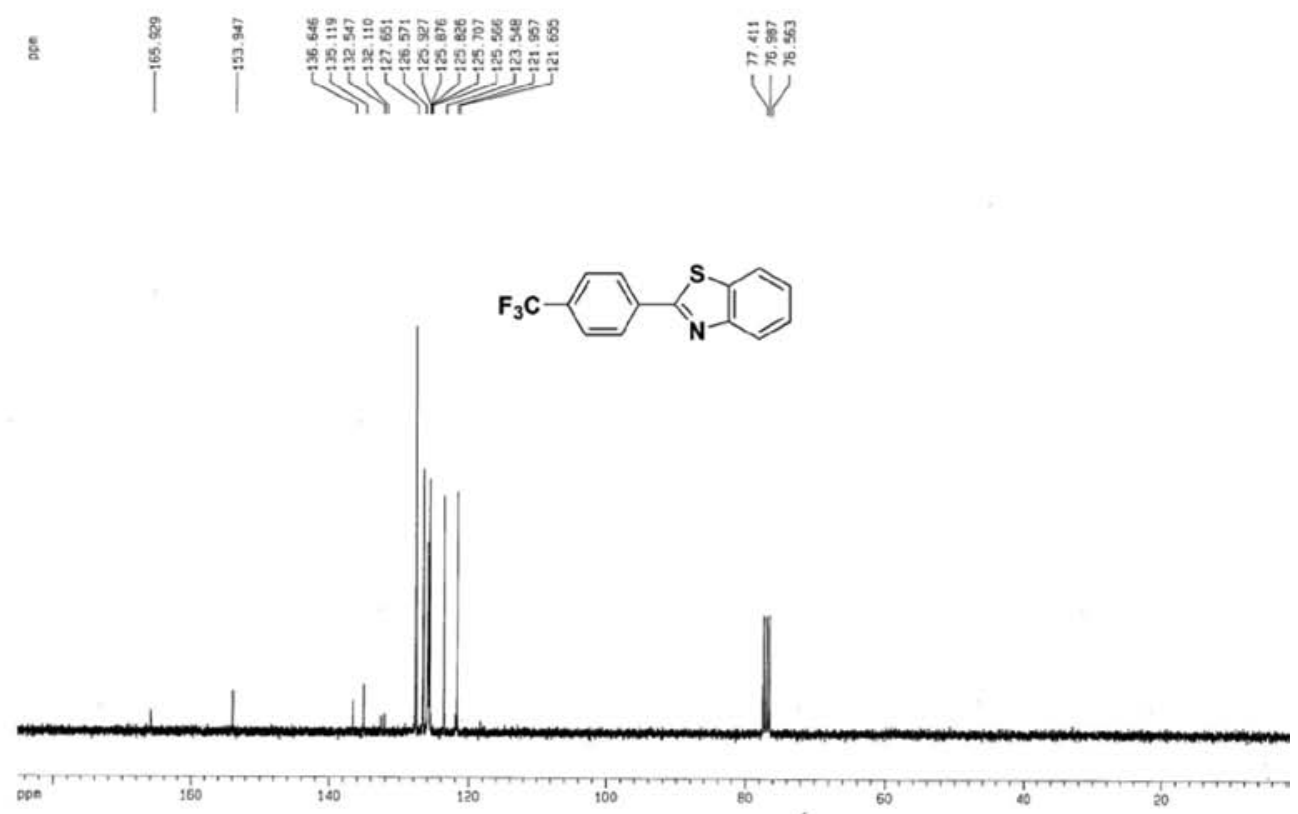

Figure S19. ${ }^{1} \mathrm{H} \mathrm{NMR}$ of $3 \mathbf{s}\left(300 \mathrm{MHz}, \mathrm{CDCl}_{3}\right)$ and ${ }^{13} \mathrm{C} \mathrm{NMR}$ of $3 s\left(75 \mathrm{MHz}, \mathrm{CDCl}_{3}\right)$. 

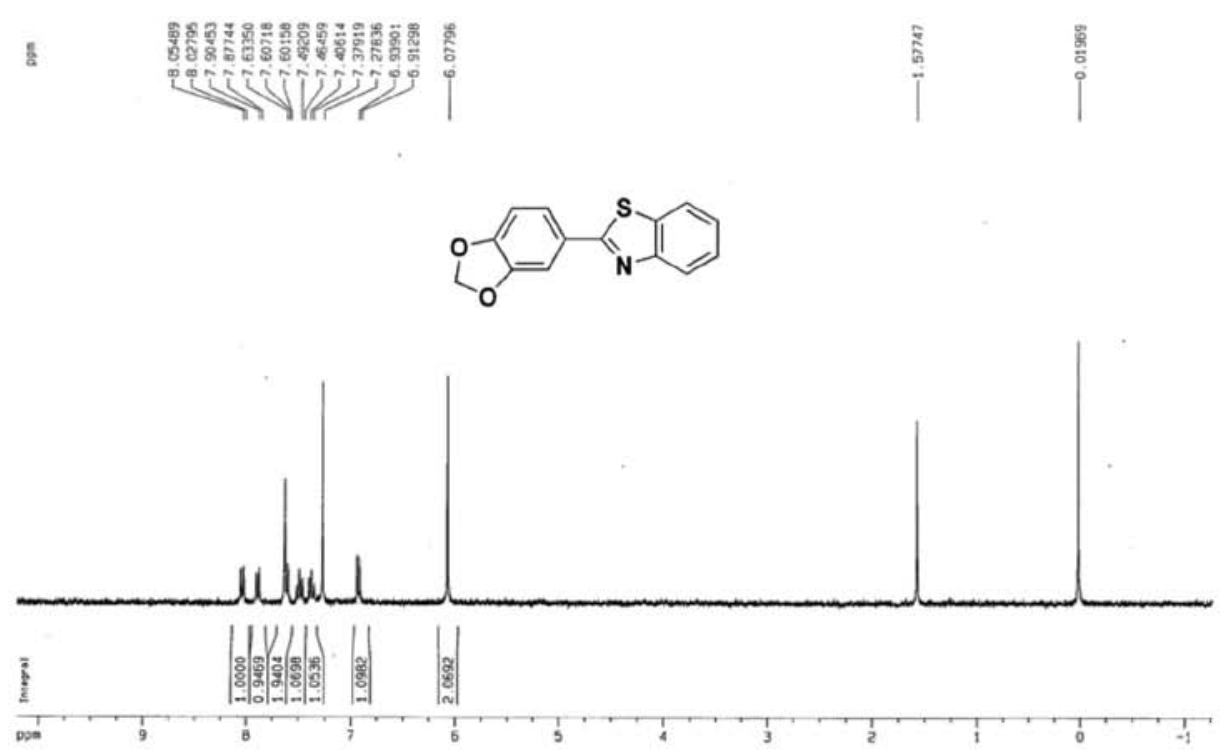

๕ू
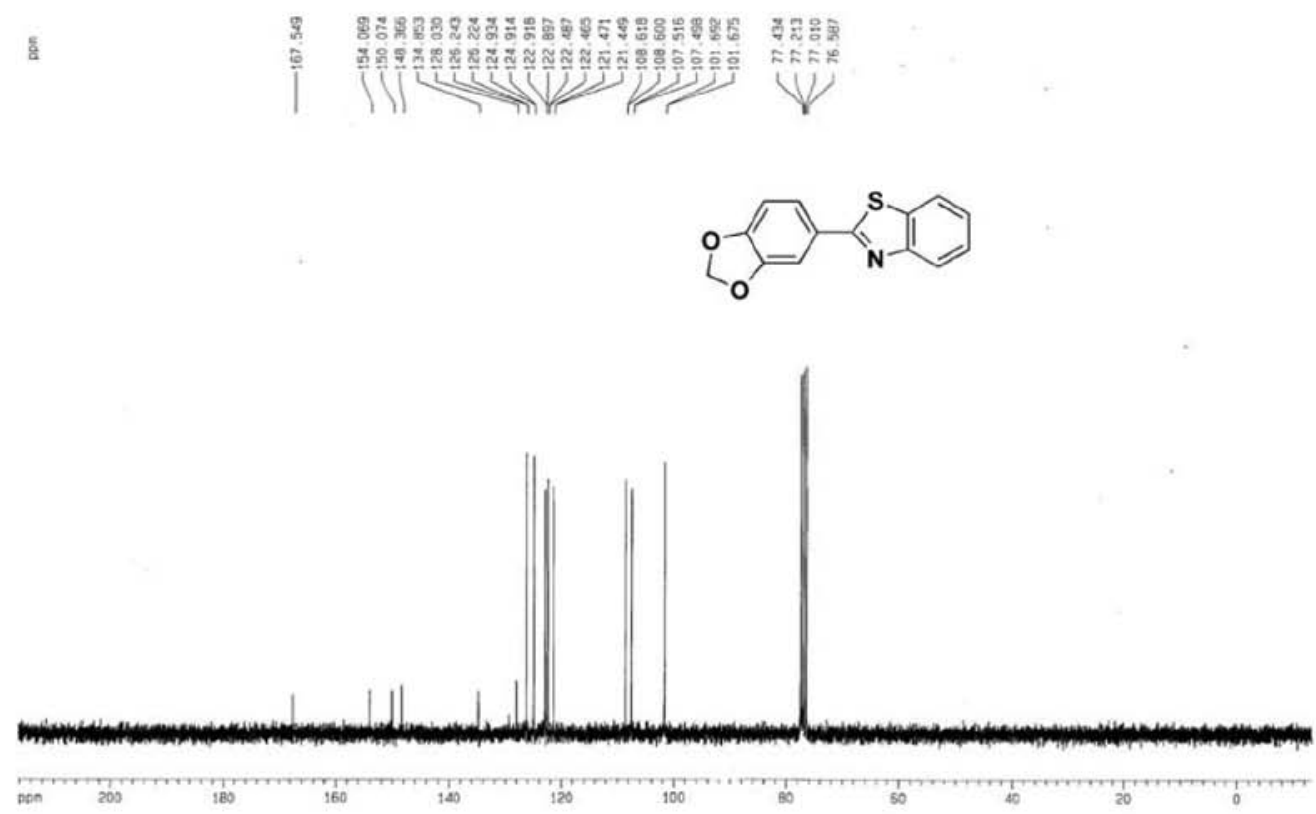

Figure S20. ${ }^{1} \mathrm{H}$ NMR of $\mathbf{3 t}\left(300 \mathrm{MHz}, \mathrm{CDCl}_{3}\right)$ and ${ }^{13} \mathrm{C} \mathrm{NMR}$ of $\mathbf{3 t}\left(75 \mathrm{MHz}, \mathrm{CDCl}_{3}\right)$. 


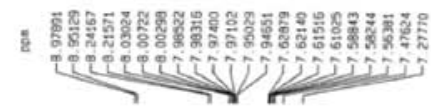

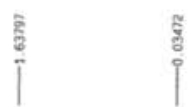

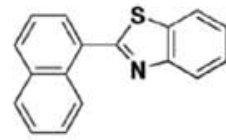

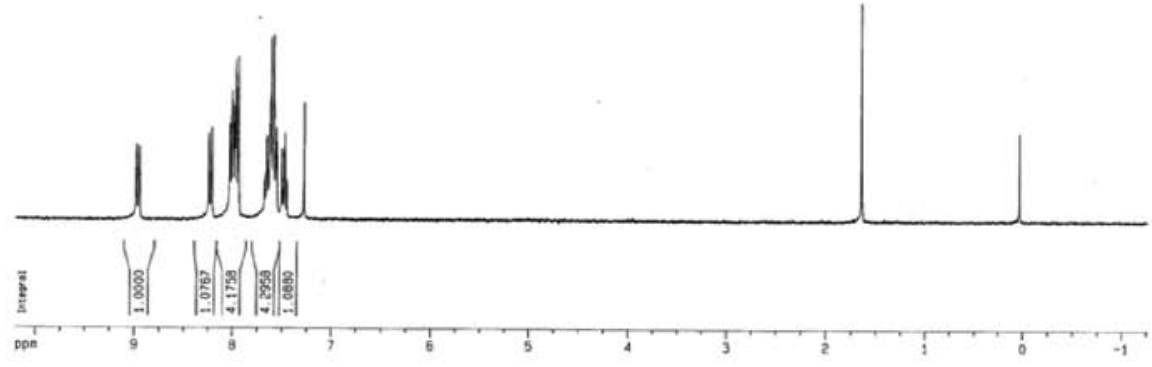

a
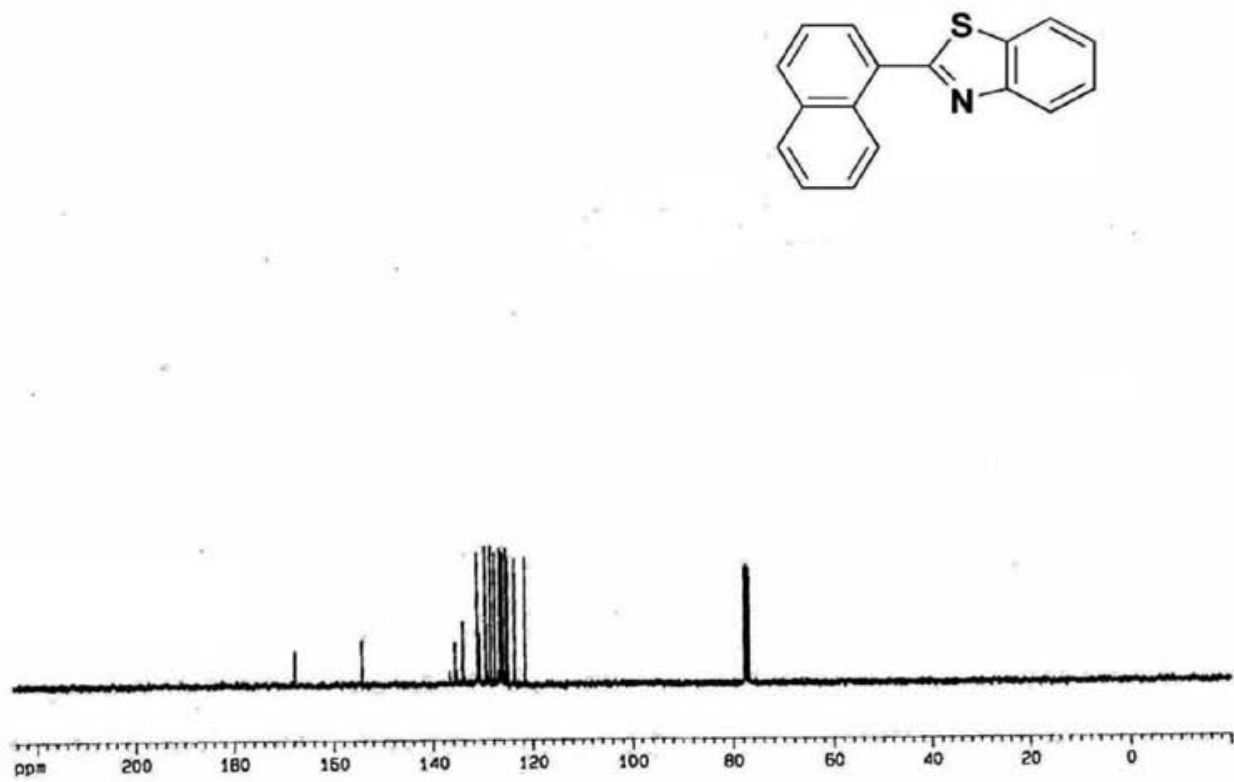

Figure S21. ${ }^{1} \mathrm{H}$ NMR of $3 \mathbf{u}\left(300 \mathrm{MHz}, \mathrm{CDCl}_{3}\right)$ and ${ }^{13} \mathrm{C} \mathrm{NMR}$ of $3 \mathbf{u}\left(75 \mathrm{MHz}, \mathrm{CDCl}_{3}\right)$. 


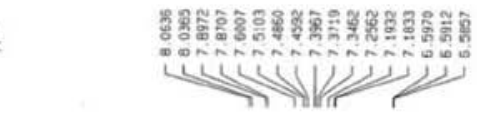<smiles>c1coc(-c2nc3ccccc3s2)c1</smiles>

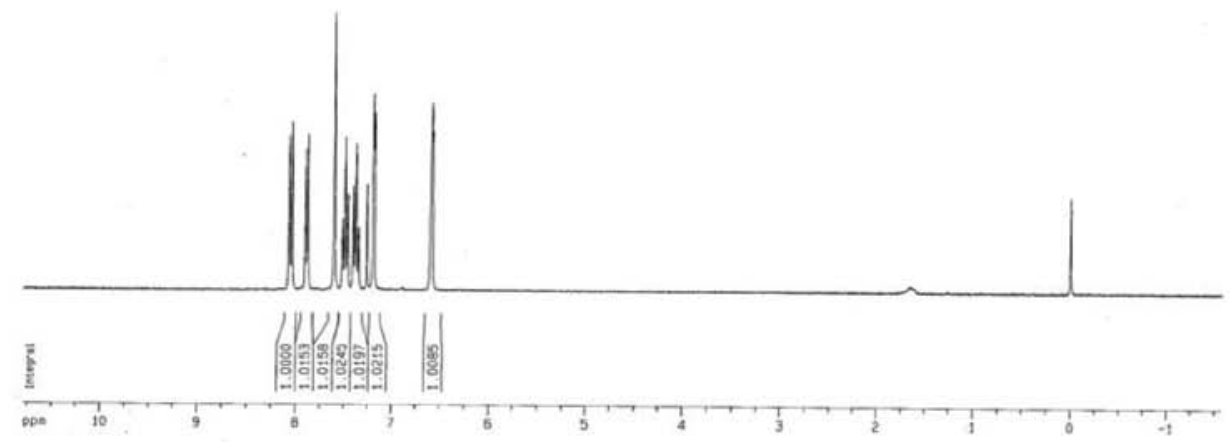

nt-1

\$
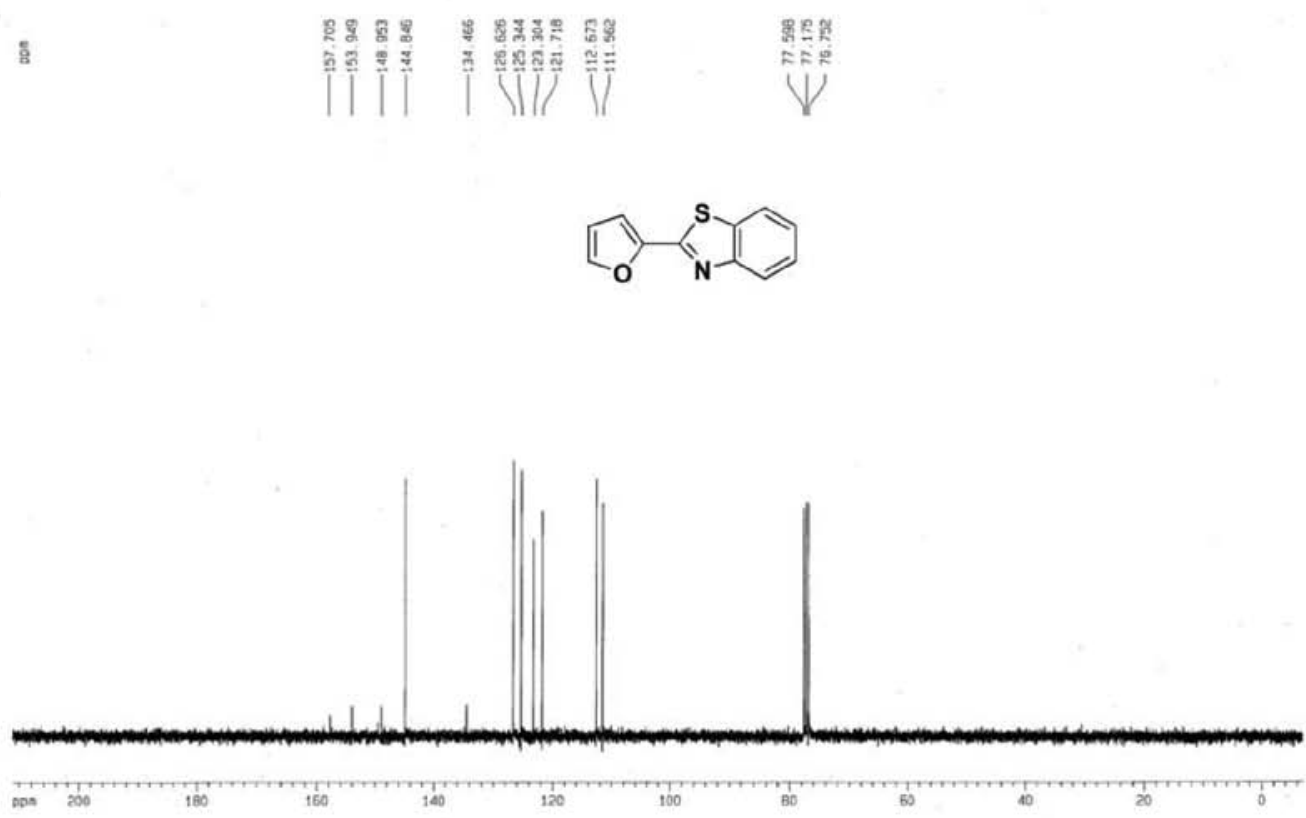

Figure S22. ${ }^{1} \mathrm{H}$ NMR of $\mathbf{3 v}\left(300 \mathrm{MHz}, \mathrm{CDCl}_{3}\right)$ and ${ }^{13} \mathrm{C} \mathrm{NMR}$ of $\mathbf{3 v}\left(75 \mathrm{MHz}, \mathrm{CDCl}_{3}\right)$. 

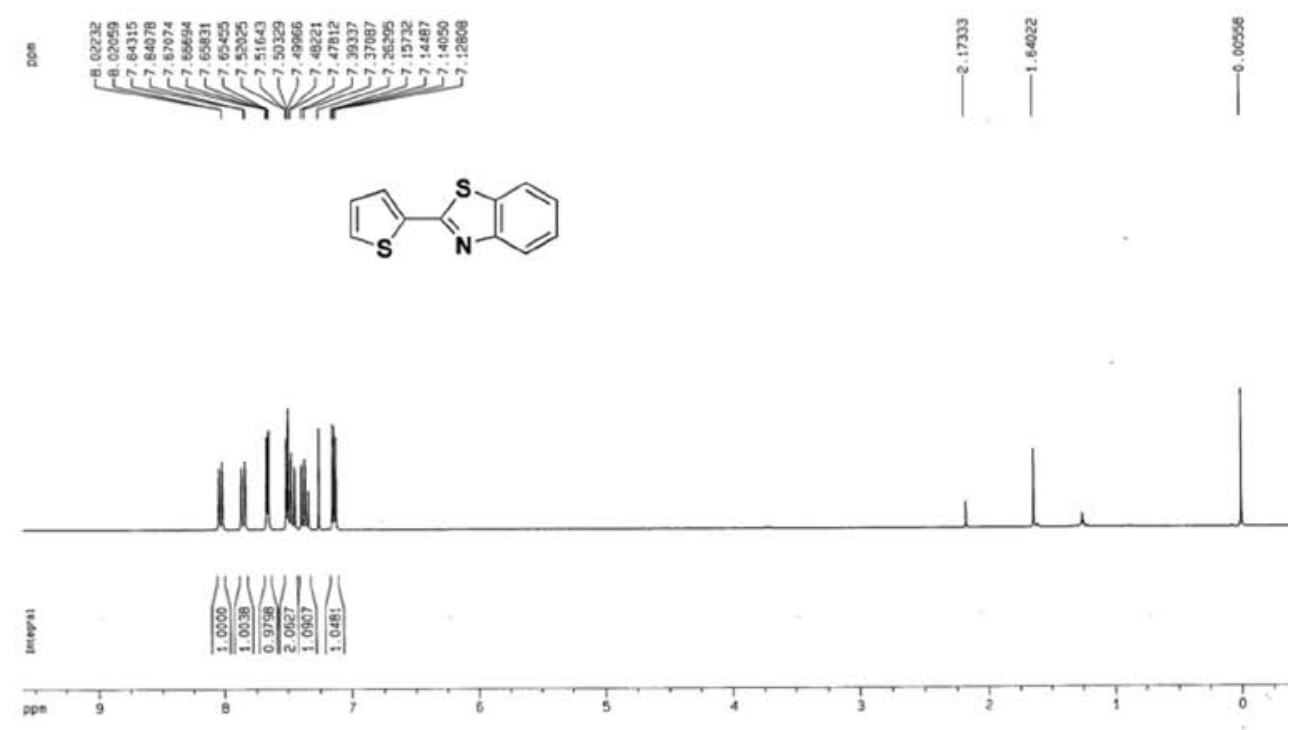

yes-3r

8
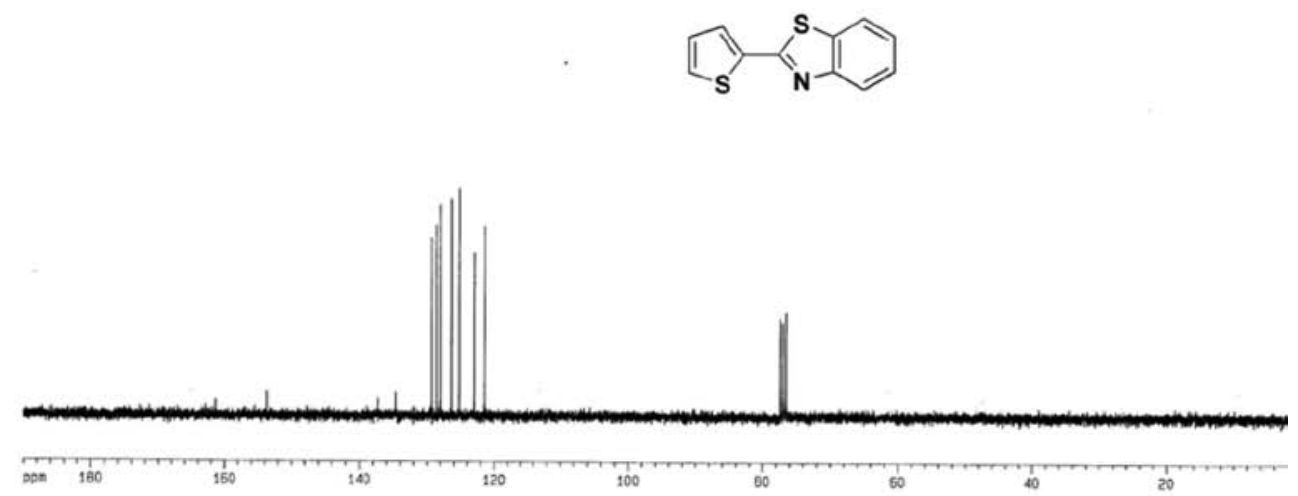

Figure S23. ${ }^{1} \mathrm{H} \mathrm{NMR}$ of $\mathbf{3 w}\left(300 \mathrm{MHz}, \mathrm{CDCl}_{3}\right)$ and ${ }^{13} \mathrm{C} \mathrm{NMR}$ of $\mathbf{3 w}\left(75 \mathrm{MHz}, \mathrm{CDCl}_{3}\right)$. 

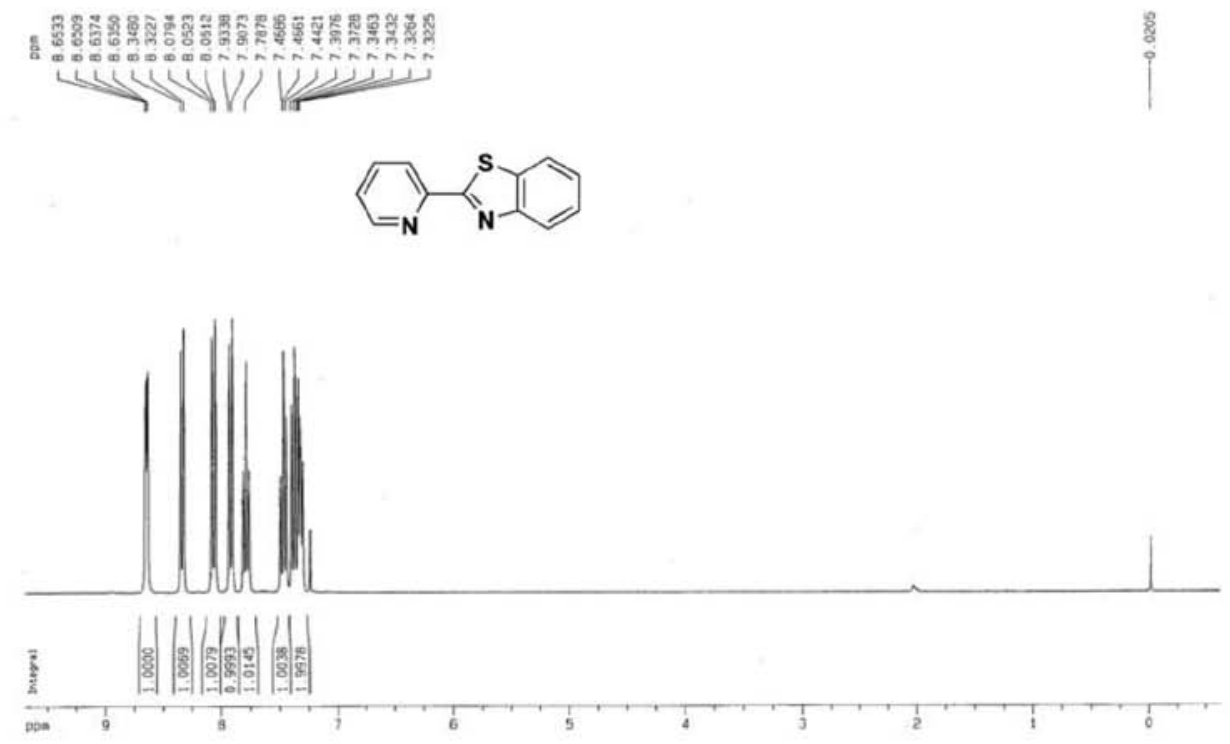

就
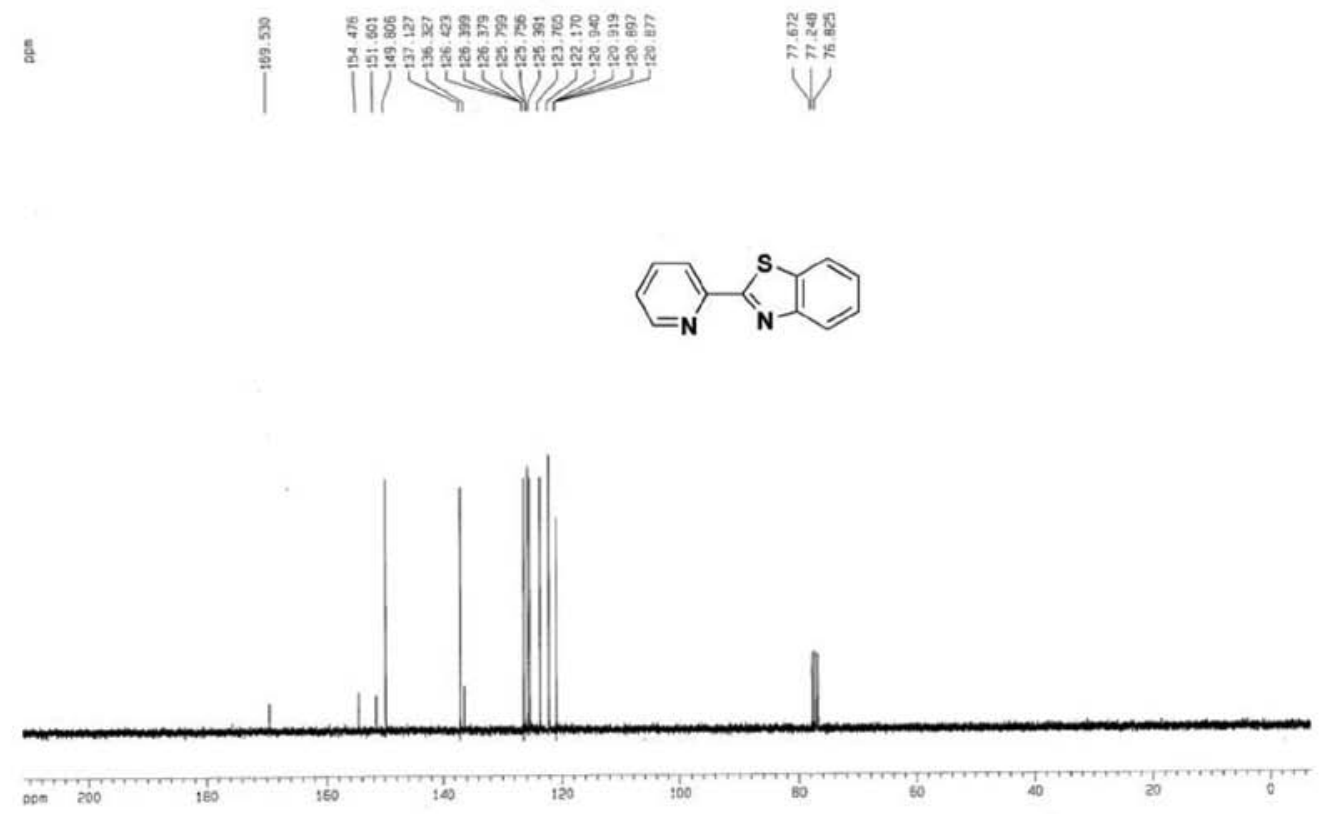

Figure S24. ${ }^{1} \mathrm{H}$ NMR of $\mathbf{3 x}\left(300 \mathrm{MHz}, \mathrm{CDCl}_{3}\right)$ and ${ }^{13} \mathrm{C} \mathrm{NMR}$ of $\mathbf{3 x}\left(75 \mathrm{MHz}, \mathrm{CDCl}_{3}\right)$. 


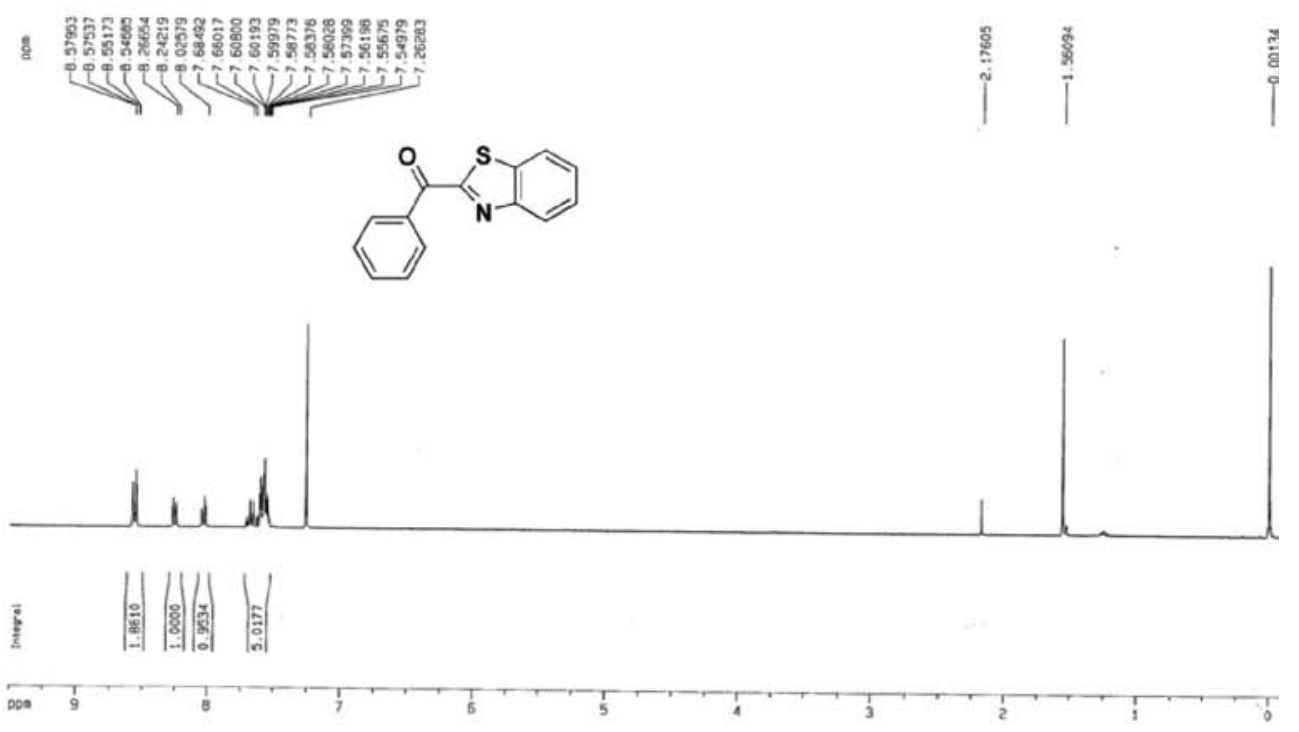

$y \times 1-3-1$

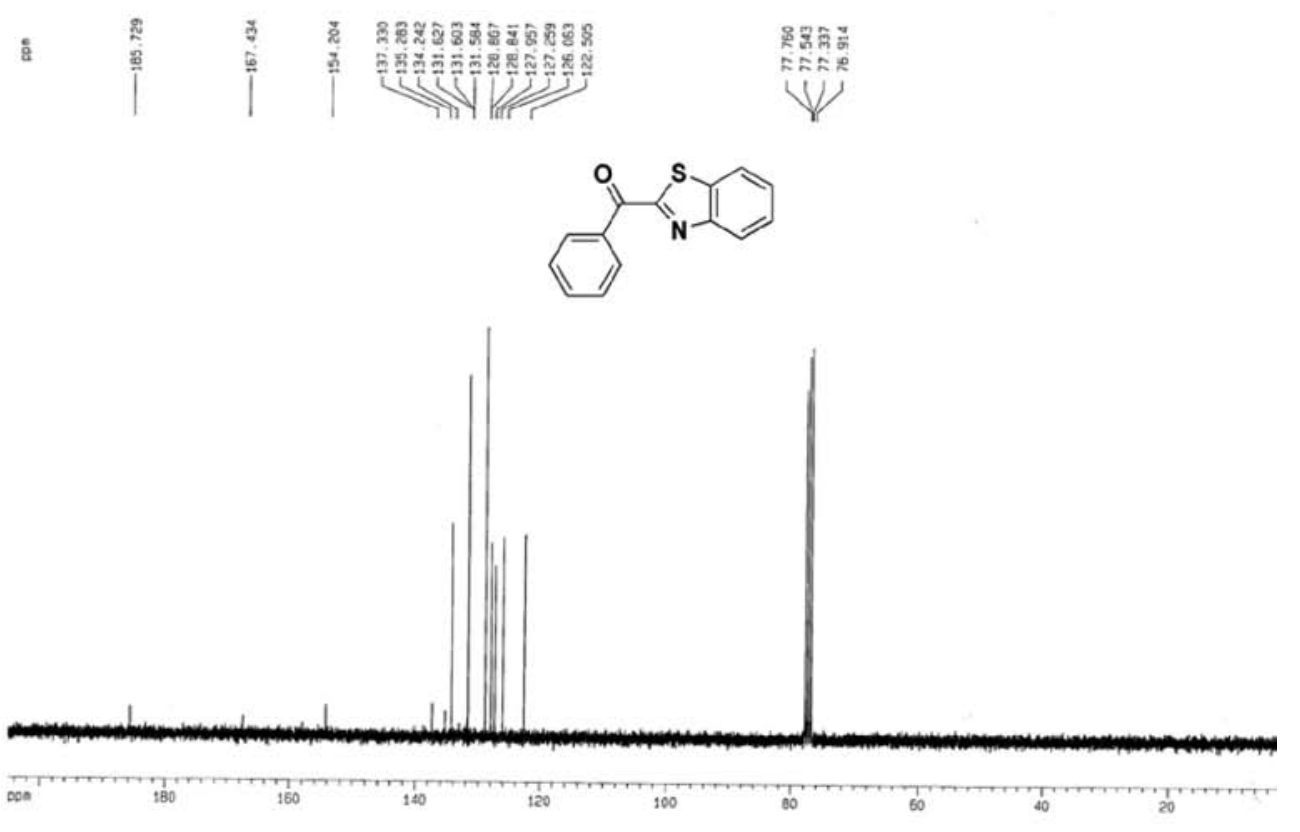

Figure S25. ${ }^{1} \mathrm{H}$ NMR of $\mathbf{4 a}\left(300 \mathrm{MHz}, \mathrm{CDCl}_{3}\right)$ and ${ }^{13} \mathrm{C} \mathrm{NMR} \mathrm{of} \mathbf{4 a}\left(75 \mathrm{MHz}, \mathrm{CDCl}_{3}\right)$. 


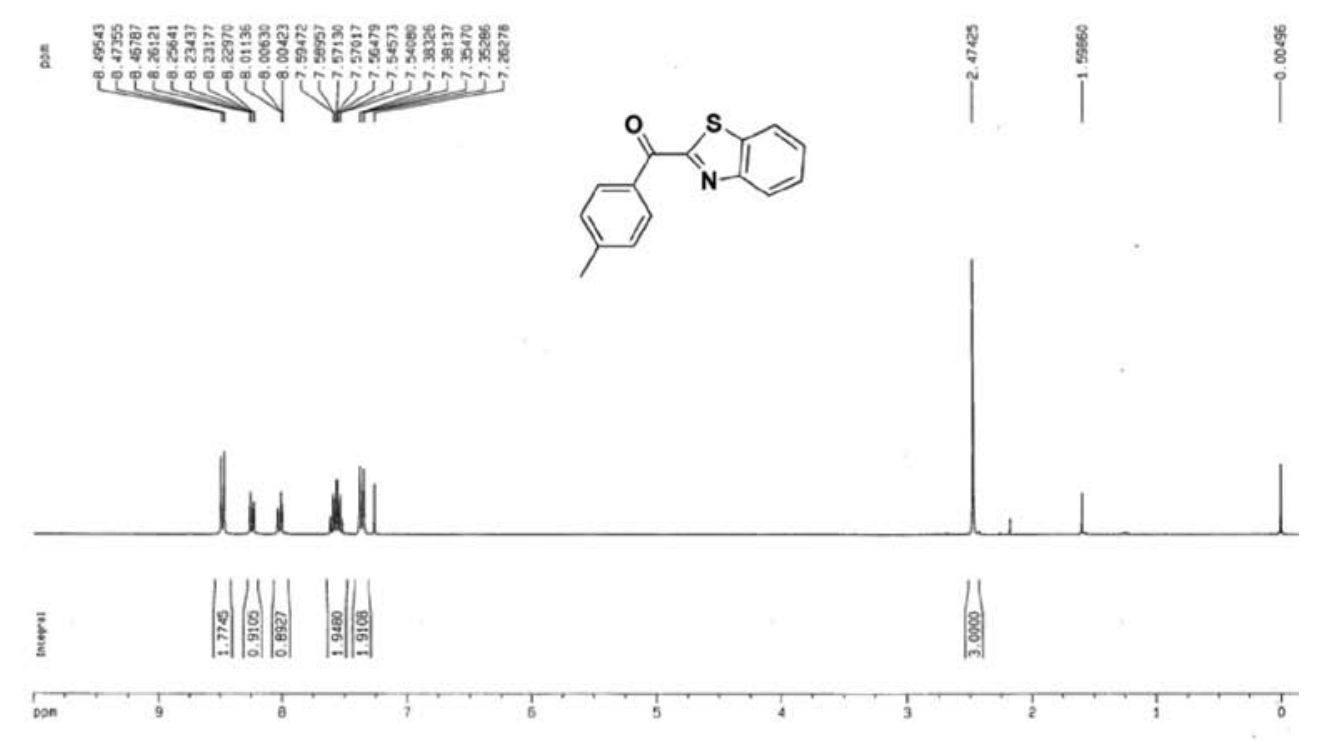

$y \times 1-3-2$

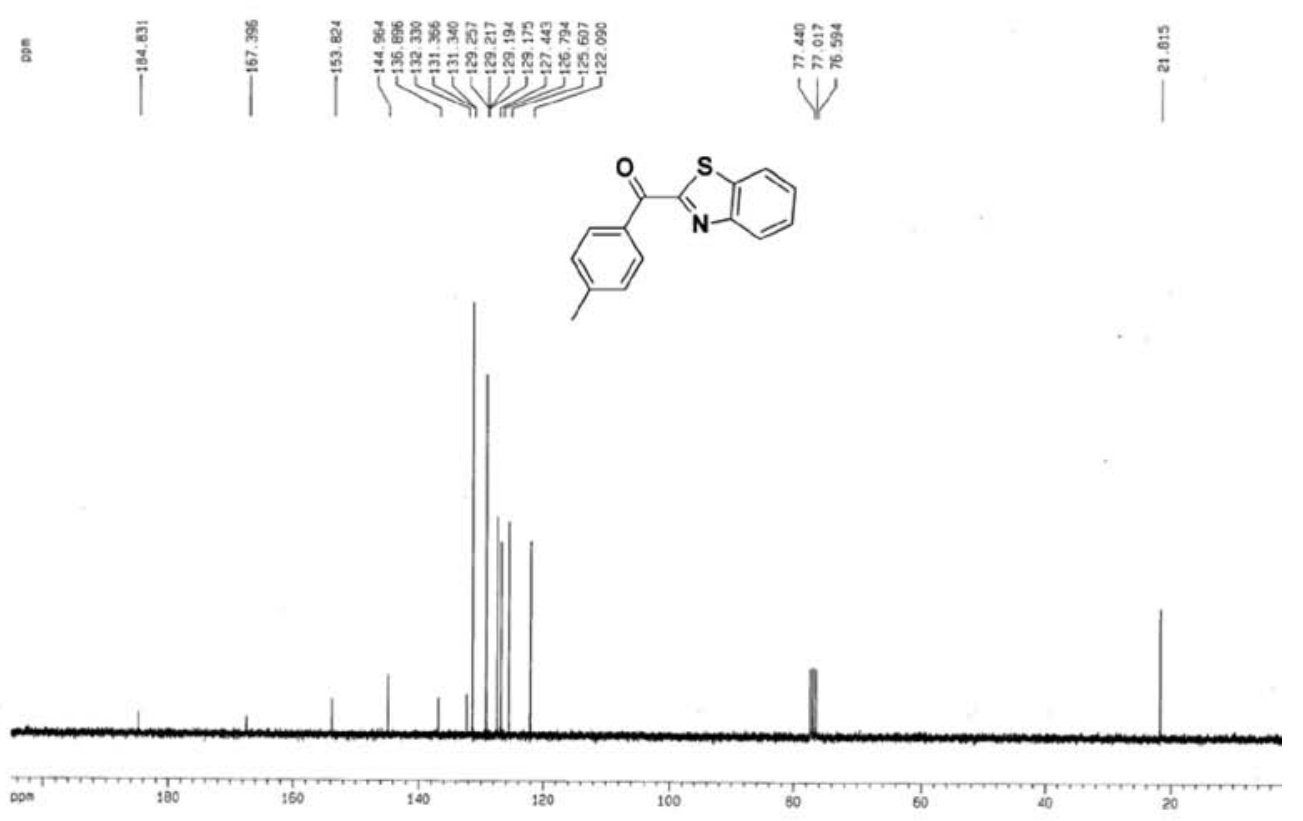

Figure S26. ${ }^{1} \mathrm{H}$ NMR of $\mathbf{4 b}\left(300 \mathrm{MHz}, \mathrm{CDCl}_{3}\right)$ and ${ }^{13} \mathrm{C} \mathrm{NMR}$ of $\mathbf{4 b}\left(75 \mathrm{MHz}, \mathrm{CDCl}_{3}\right)$. 


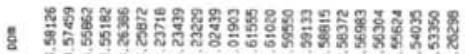

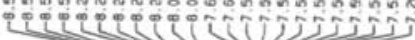

$\rightarrow$ i
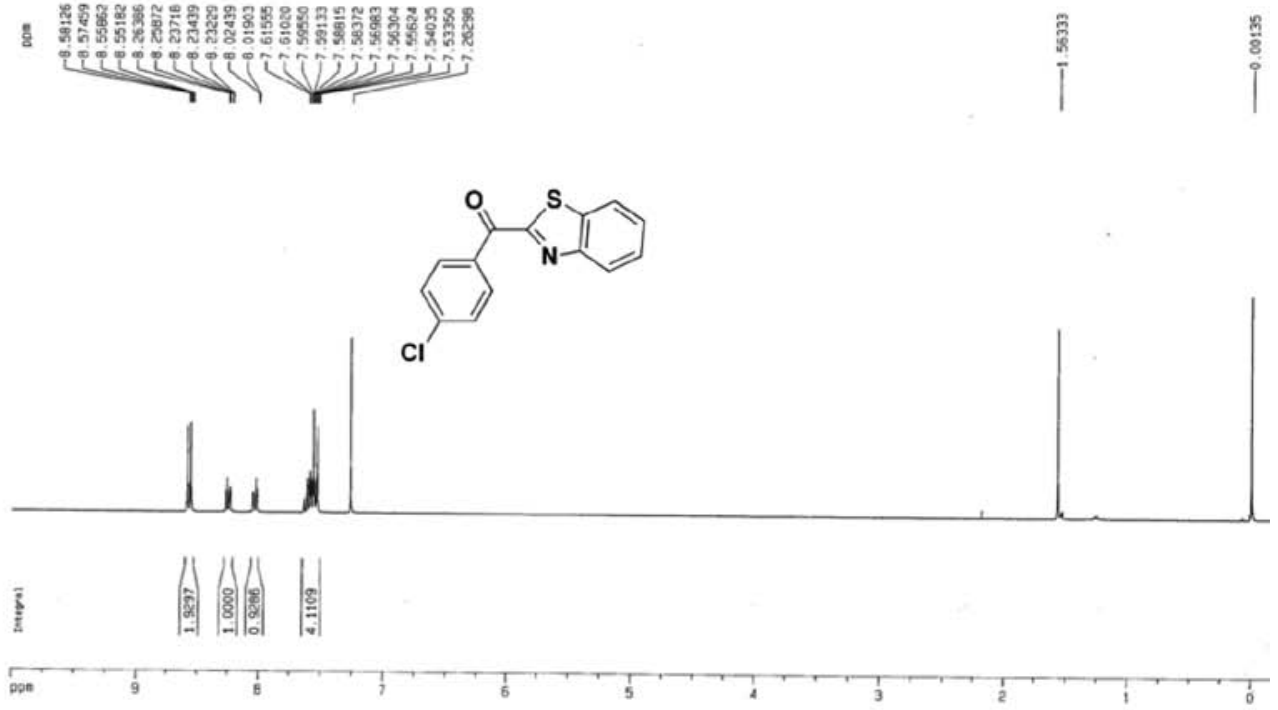

nel-3-3
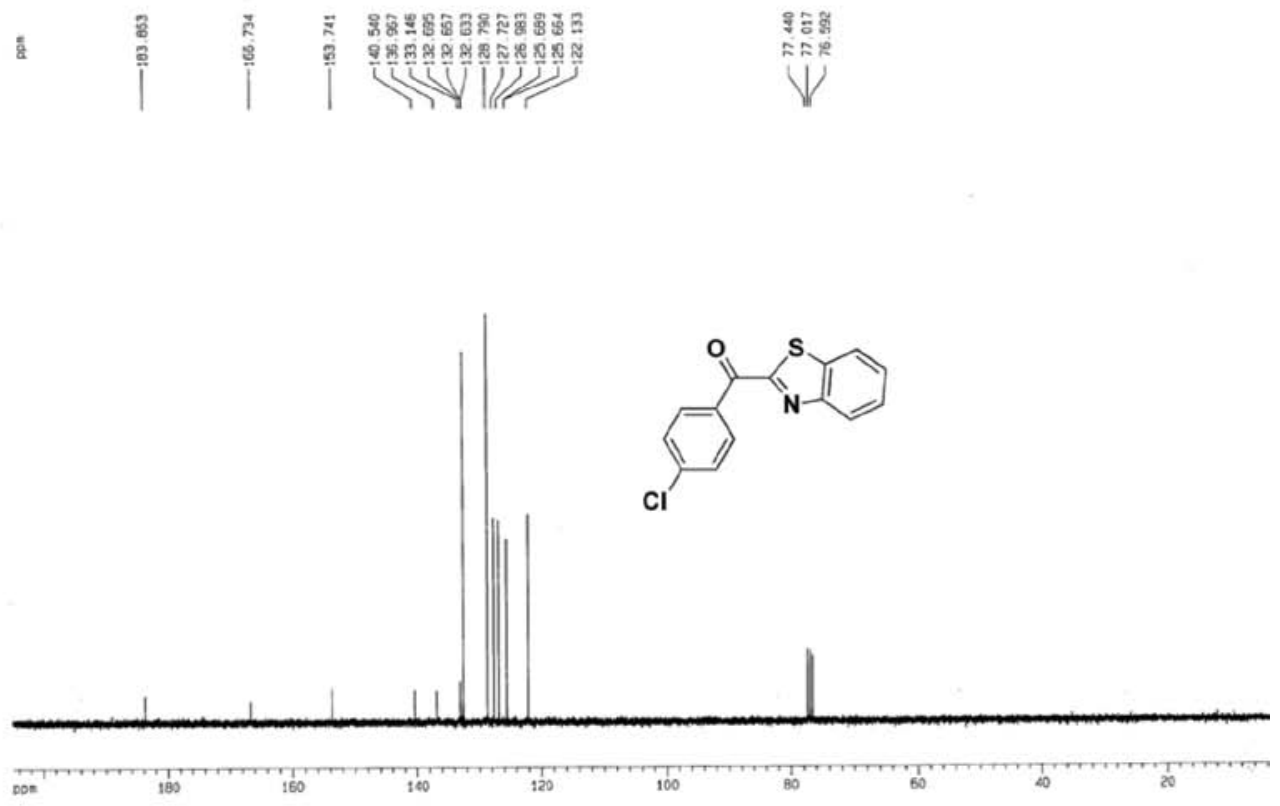

Figure S27. ${ }^{1} \mathrm{H}$ NMR of $4 \mathbf{c}\left(300 \mathrm{MHz}, \mathrm{CDCl}_{3}\right)$ and ${ }^{13} \mathrm{C} \mathrm{NMR}$ of $\mathbf{4 c}\left(75 \mathrm{MHz}, \mathrm{CDCl}_{3}\right)$ 


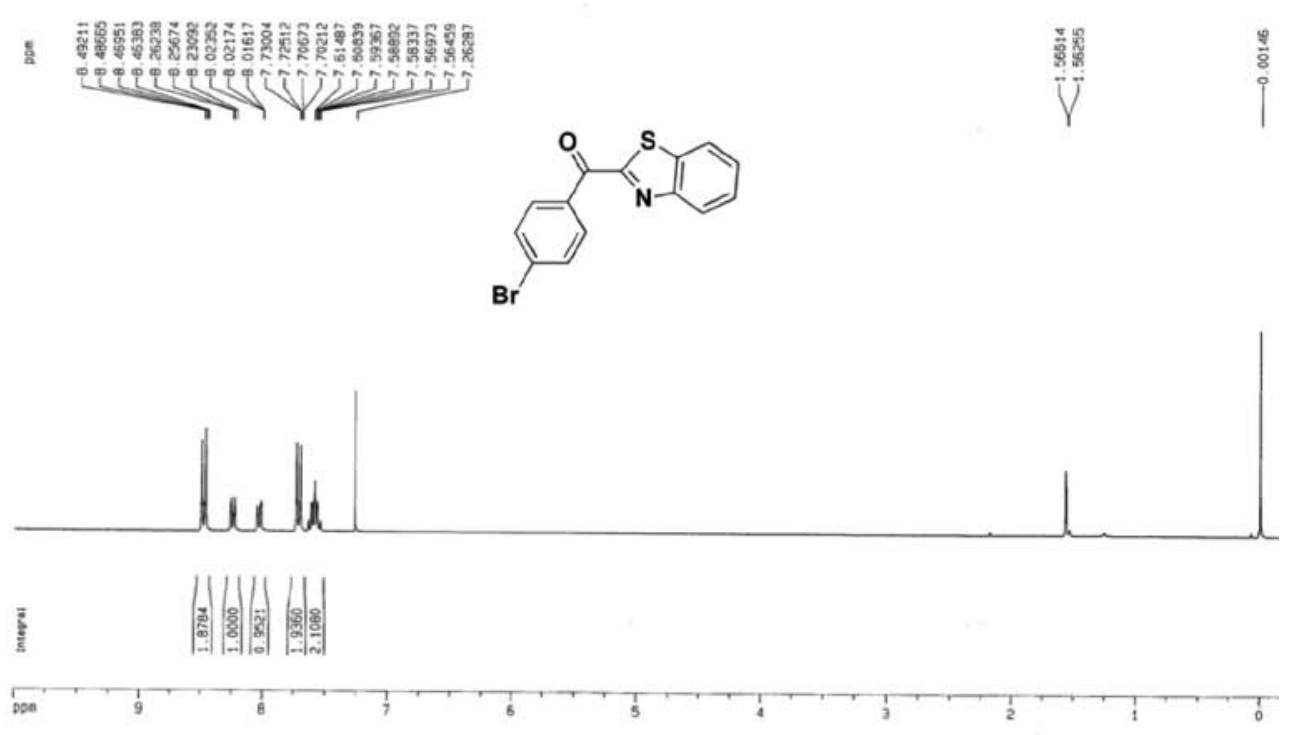

$r \times 1-3-4$
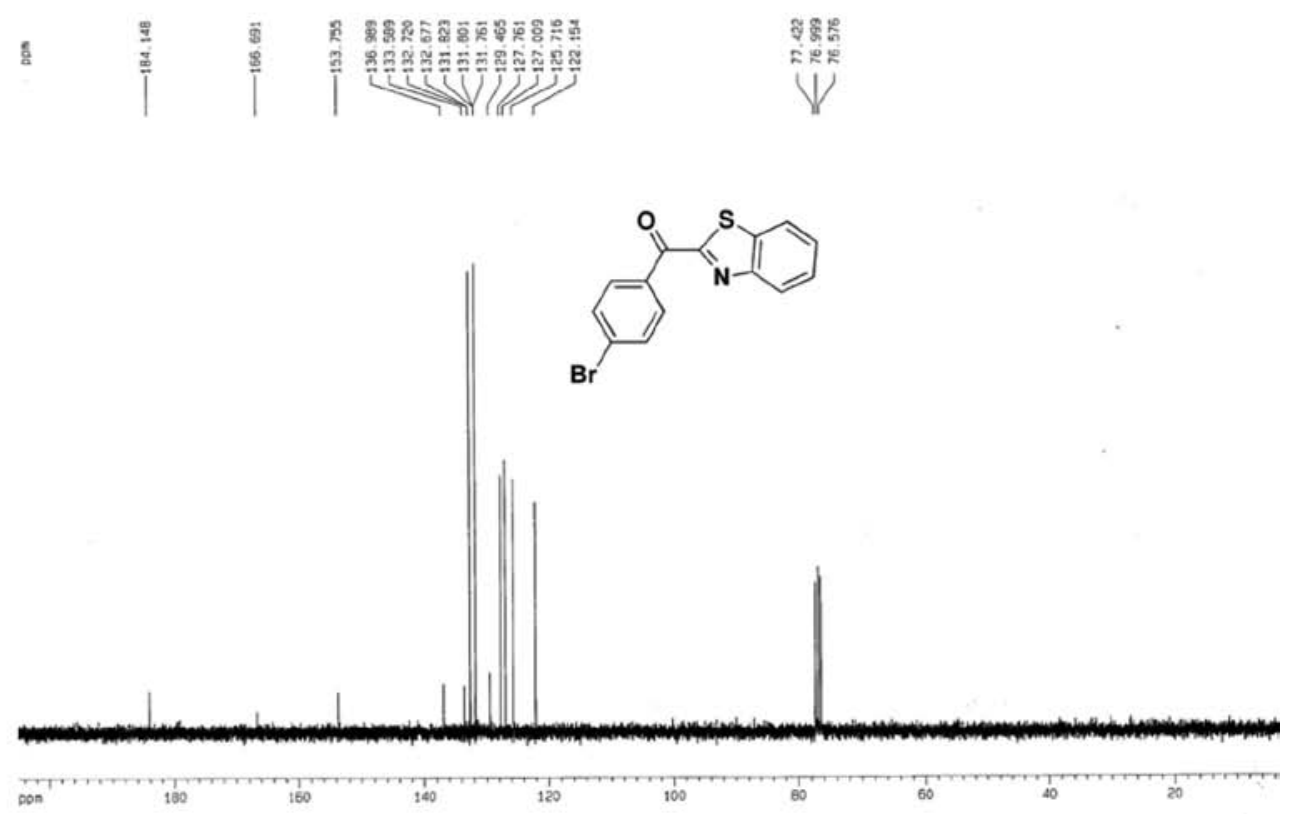

Figure S28. ${ }^{1} \mathrm{H}$ NMR of $\mathbf{4 d}\left(300 \mathrm{MHz}, \mathrm{CDCl}_{3}\right)$ and ${ }^{13} \mathrm{C} \mathrm{NMR}$ of $\mathbf{4 d}\left(75 \mathrm{MHz}, \mathrm{CDCl}_{3}\right)$. 